\title{
Geochemical Data for 95 Thermal and Nonthermal Waters of the Valles Caldera Southern Jemez Mountains Region, New Mexico
}

Fraser Goff

Tamsin McCormick*

Pat E. Trujillo, Jr.

Dale Counce

C. O. Grigsby

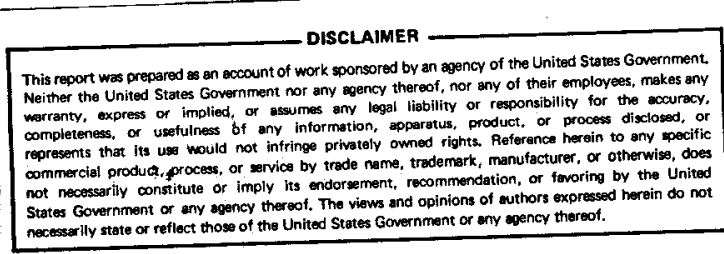

-Graduate Research Assistant. Geology Department, Arizona State University, Tempe, AZ 85287. 


\section{DISCLAIMER}

This report was prepared as an account of work sponsored by an agency of the United States Government. Neither the United States Government nor any agency Thereof, nor any of their employees, makes any warranty, express or implied, or assumes any legal liability or responsibility for the accuracy, completeness, or usefulness of any information, apparatus, product, or process disclosed, or represents that its use would not infringe privately owned rights. Reference herein to any specific commercial product, process, or service by trade name, trademark, manufacturer, or otherwise does not necessarily constitute or imply its endorsement, recommendation, or favoring by the United States Government or any agency thereof. The views and opinions of authors expressed herein do not necessarily state or reflect those of the United States Government or any agency thereof. 


\section{DISCLAIMER}

Portions of this document may be illegible in electronic image products. Images are produced from the best available original document. 
GEOCHEMICAL DATA FOR 95 THERMAL AND NONTHERMAL WATERS OF THE VALLES CALDERA--SOUTHERN JEMEZ MOUNTAINS REGION, NEW MEXICO

by

Fraser Goff, Tamsin McCormick, Pat E. Trujillo, Jr., Dale Counce, and C. 0 . Grigsby

\section{ABSTRACT}

This report presents field, chemical, and isotopic data for 95 thermal and nonthermal waters of the southern Jemez Mountains, New Mexico. This region includes all thermal and mineral waters associated with Valles Caldera and many of those located near the Nacimiento Uplift, near San Ysidro. Waters of the region can be categorized into five general types: (1) surface and nearsurface meteoric waters; (2) acid-sulfate waters (Valles Caldera); (3) thermal meteoric waters (Valles Caldera); (4) deep geothermal and derivative waters (Valles Caldera); and (5) mineralized waters near San Ysidro. Some waters display chemical and isotopic characteristics intermediate between the types listed. The object of the data is to help interpret geothermal potential of the Jemez Mountains region and to provide background data for investigating problems in hydrology, structural geology, hydrothermal alterations, and hydrothermal solution chemistry.

\section{INTRODUCTION}

The Jemez Mountains consist of volcanic rocks of basaltic to rhyolitic composition that overlie Tertiary to Paleozoic sediments on the western margin of the Rio Grande rift. Volcanic activity culminated in the Pleistocene with eruption of $\backsim 500 \mathrm{~km}^{3}$ of Bandelier tuff and with formation of the Valles Caldera, a large well-preserved silicic cauldron. The valles region also contains a variety of hot springs having distinct geologic, chemical, and 
isotopic characteristics. Young, large silicic volcanic centers such as the Valles Caldera have great potential for geothermal energy because they overlie shallow magma reservoirs of batholithic proportions. A study of the chemistry of geothermal fluids can provide information on the hydrothermal systems and the geologic formations and structures through which they flow. The purpose of this paper is to present field, chemical, and isotopic data for ground water and thermal water in the Jemez Mountains area, to characterize different water types and relate them to the hydrothermal systems. These data are presented to aid the overall assessment of geothermal resources of the Jemez Mountains and to provide data for other scientific investigations.

The general geohydrology and geochemistry of waters in the Jemez Mountains have been described by Goff and Grigsby, ${ }^{1}$ Goff et al., ${ }^{2}$ Goff and Sayer, ${ }^{3}$ Trainer and Lyford, ${ }^{4}$ Trainer, ${ }^{5,6}$ Titus, ${ }^{7}$ Purtymun and Johansen, ${ }^{8}$ Purtymun et al., 9,10 and Purtymun. 11

\section{SIMPLIFIED GEOLOGY}

The geology of the Jemez Mountains has been described by Ross et al., 12 Smith et al., ${ }^{13}$ Griggs, ${ }^{14}$ Doell et al., ${ }^{15}$ and Bailey et al. ${ }^{16}$ Smith, Bailey and Ross ${ }^{17}$ published an excellent regional geologic map in 1970 . The Jemez Mountains consist of an extensive pile of Tertiary and Quaternary lavas and tuffs (10 to $0.1 \mathrm{Myr}$ ) overlying Precambrian granite, gneiss, and schist and the Paleozoic to Mesozoic sedimentary sequence of the Colorado Plateau (Figs. 1 and 2). The main Paleozoic units include the Pennsylvanian Madera Formation, the red sandstone, siltstone, and shale of the Permian Abo and Yeso Formations and the Triassic Chinle Formation. Mesozoic rocks crop out south of the Jemez Mountains near San Ysidro. The older Mesozoic units include the Entrada Formation sandstone, Todilto Formation evaporites (mainly gypsum), and Morrison Formation shale and sandstone.

Colorado Plateau rocks of this region are down-faulted to the east into the Rio Grande rift. Unconsolidated Tertiary sediments of the Santa Fe Formation thicken eastward towards the axis of the rift. The Jemez Mountains volcanics occur at the intersection of the rift with the northeast-trending Jemez Lineament, a line of Miocene to Quaternary volcanic fields extending across the northwest portion of New Mexico. ${ }^{18-20}$

Volcanic activity commenced with dominantly mafic to intermediate lava flows that are partly interbedded with the Tertiary sediments. These rocks 


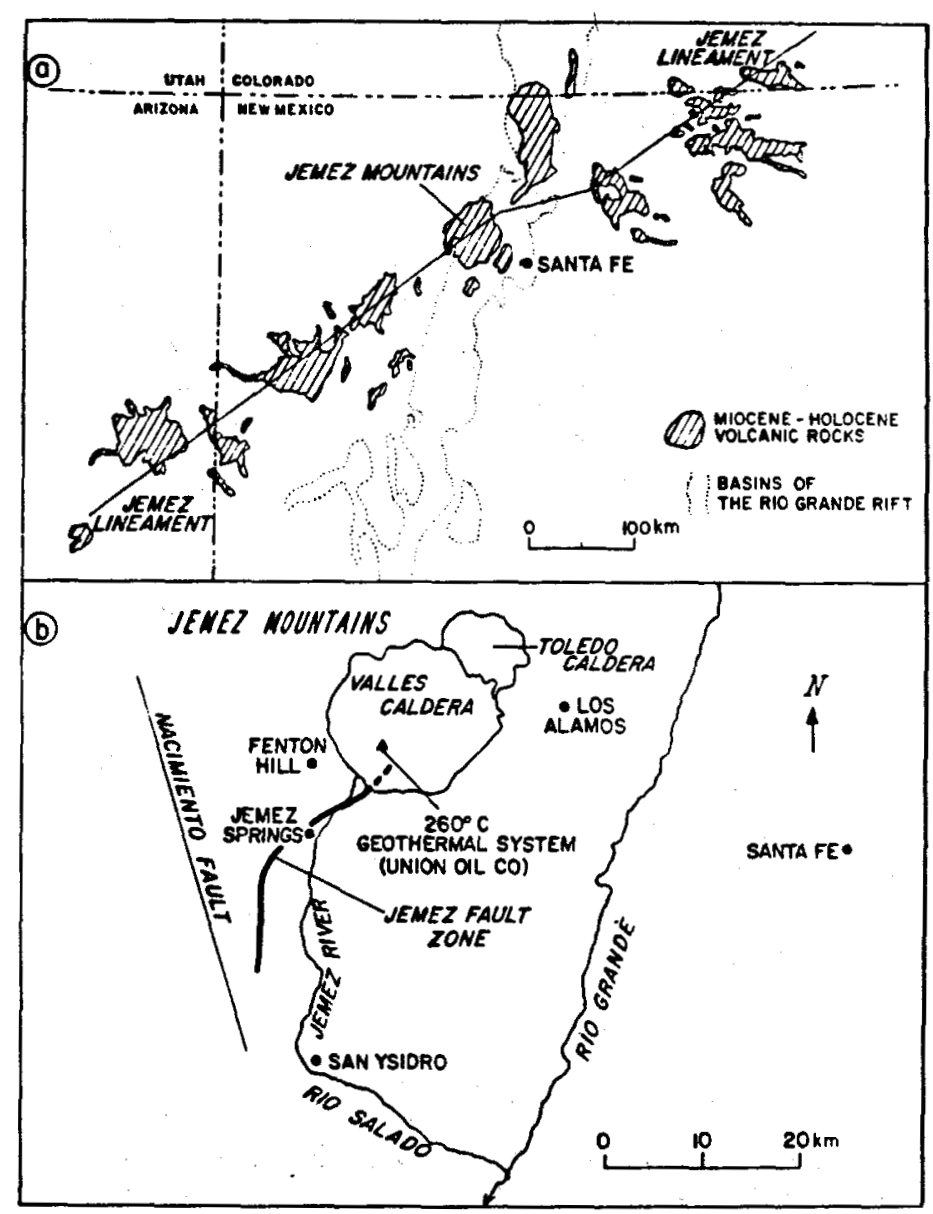

Fig. 1.

Location map showing (a) volcanic centers in relationship to the Rio Grande rift and Jemez Lineament and (b) sketch of Jemez Mountains region.

are best exposed in the areas north, northeast, and south of the valles Caldera complex. Two major eruptions of Bandelier rhyolite tuff in the early Pleistocene resulted in the formation of the Toledo and Valles Caldera. Deposits of tuff up to $300 \mathrm{~m}$ thick occur to the west and east of the caldera forming the Jemez and Pajarito Plateaus, respectively. The final activity in the Jemez Mountains involved eruption of rhyolite domes, obsidian, and tuffs in the moat zone of the Valles Caldera.

\section{METHODS AND PROCEDURES FOR COLLECTION AND ANALYSIS OF WATERS}

Temperatures were recorded with mercury thermometers and field $\mathrm{pH}$ was determined using a $\mathrm{pH}$ meter or using sensitive limited range $\mathrm{pH}$ test papers (Colorfast Indicator Strips nos. 9581, 9582, and 9583). Laboratory values of $\mathrm{pH}$ are not considered reliable because most waters gain or lose $\mathrm{CO}_{2}$ gas after 


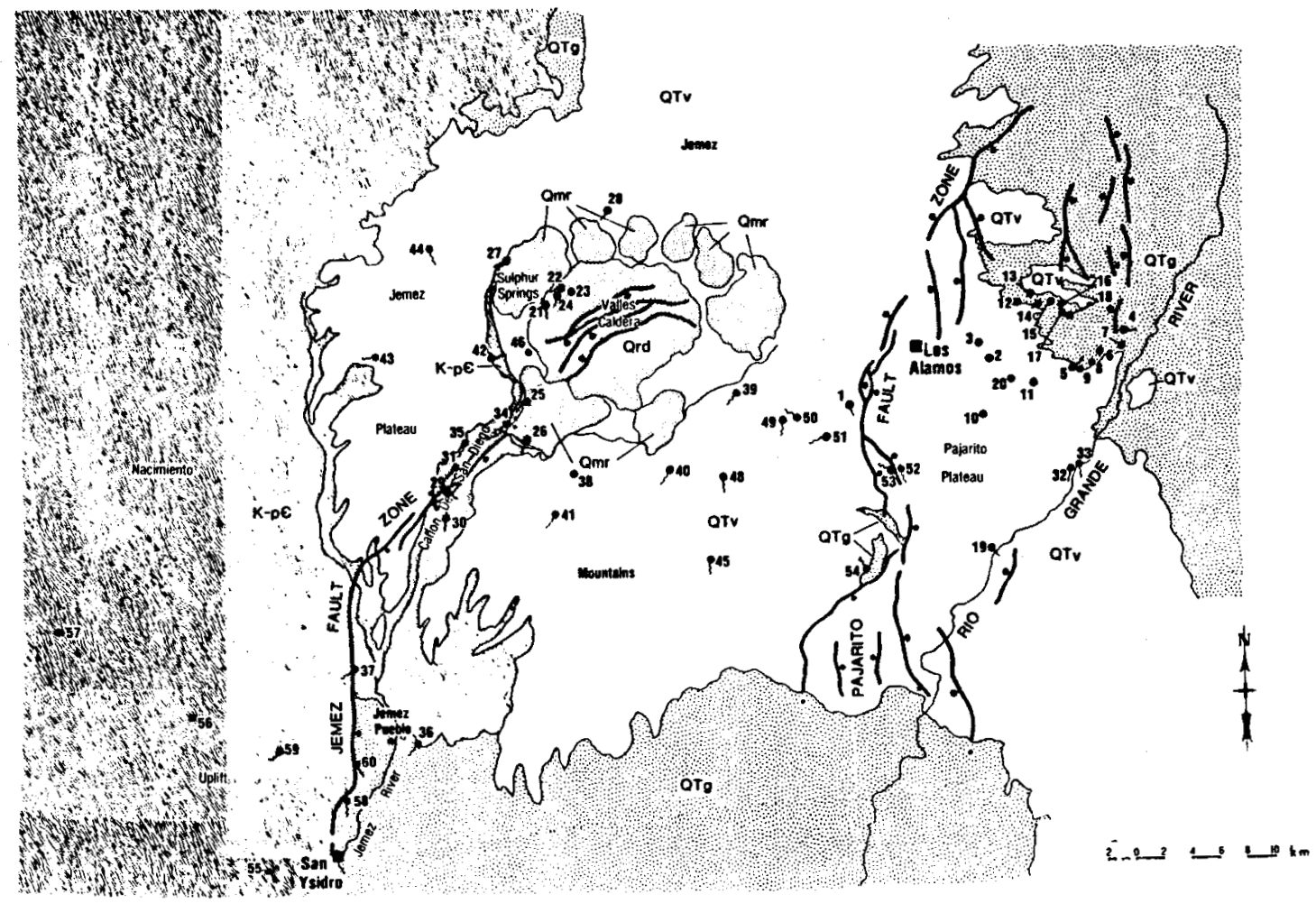

Fig. 2.

Schematic geologic map of Jemez Mountains region showing major faults. Numbers refer to spring and well locations listed in tables in Appendix B. Qrd = Resurgent Dome of Valles Caldera, Qmr = Moat Rhyolites of Valles Caldera, QTV = Quaternary-Tertiary volcanic rocks, QTg = Quaternary-Tertiary sediments, $K-p G=$ Cretaceous-Precambrian bedrock.

sampling and before laboratory analysis. This alters the concentration of bicarbonate ions, which in turn changes the $\mathrm{pH}$. Flow rates of springs were estimated visually; flow rates of wells were obtained from gauges on the wellhead where possible. A compilation of unusual springs of the Jemez Mountains is presented in Appendix A. Field data is recorded in Table B-I (all tables appear in Appendix B).

Samples of water for chemical analysis were filtered using a large syringe attached to a filter holder containing $0.8 \mu$ filter paper. The filtered water was squirted brimful into polyethylene bottles with Polyseal caps. Three types of samples were collected: (1) a 500-me bottle of filtered unacidified water for anions, (2) a 250-me bottle of filtered acidified water for cations, and (3) a 125-me bottle of filtered diluted water for silica. Dilute HCl was added dropwise to the acidified sample until the pH was less than 2. The bottles used for silica analyses contained $90 \mathrm{me}$ of deionized 
water before $10 \mathrm{me}$ of sample were added. This dilution prevents polymerization of monomeric silica in more concentrated water samples before analysis. Samples for determination of $A l$ were collected and analyzed according to our modified procedure of Barnes. ${ }^{21}$ Major element analyses are presented in Table B-II.

Laboratory analyses were performed by the following methods: $\mathrm{SiO}_{2}$ by a colorimetric method using a yellow molybdate complex; $\mathrm{Fe}, \mathrm{Mn}, \mathrm{Ca}, \mathrm{Mg}, \mathrm{Na}, \mathrm{K}$, and $\mathrm{Li}$ by atomic absorption spectroscopy; $\mathrm{HCO}_{3}$ by sulfuric acid titration; $\mathrm{SO}_{4}$ and $\mathrm{Cl}$ by ion chromatography; $\mathrm{F}$ by either selective ion electrode or ion chromatography; and $\mathrm{B}$ by colorimetry using azomethine-H. Because $\mathrm{HCO}_{3}$ was not determined in the field, $\mathrm{HCO}_{3}$ values listed in Table $\mathrm{B}-\mathrm{II}$ may not be reliable for dilute (unbuffered) waters. Analyses of $\mathrm{Ag}, \mathrm{Ba}, \mathrm{Cd}, \mathrm{Cr}, \mathrm{Cu}, \mathrm{Mo}, \mathrm{Ni}, \mathrm{Pb}$, $\mathrm{Sr}$, and $\mathrm{Zn}$ were performed by atomic absorption spectroscopy either by using a graphite furnace or flame excitation. These data appear in Table B-III.

Samples for $D$ and ${ }^{18} 0$ analysis were collected by filling $125-\mathrm{me}$ glass bottles full of raw water and sealing with a Polyseal cap. Isotope variations were determined by standard methods and the data appear in Table B-IV.

IV. GEOHYDROLOGY AND GEOCHEMISTRY

The waters described in this report can be divided into several groups on the basis of field, chemical, and isotopic characteristics. In this section each group is discussed separately, but the reader should refer to Figs. 6 through 13 to observe chemical and isotopic differences and similarities among water types.

A. Surface and Near-Surface Meteoric Water - Los Alamos Area and Miscellaneous

Surface and near-surface meteoric waters are generally cold, potable, and dilute. Waters included in this group issue from water-supply and test wells in the Pajarito Plateau and from cold springs, creeks, and wells in the Valles Caldera-southern Jemez Mountains region (see Figs. 2-5 for locations). Cold meteoric waters occur in three different geologic settings; (1) within late Tertiary to Quaternary volcanic rocks of the Jemez Mountains volcanic field, (2) within late Tertiary basin-fill sediments of the Rio Grande rift, and (3) within Paleozoic to Mesozoic sediments of the Colorado Plateau. These three 


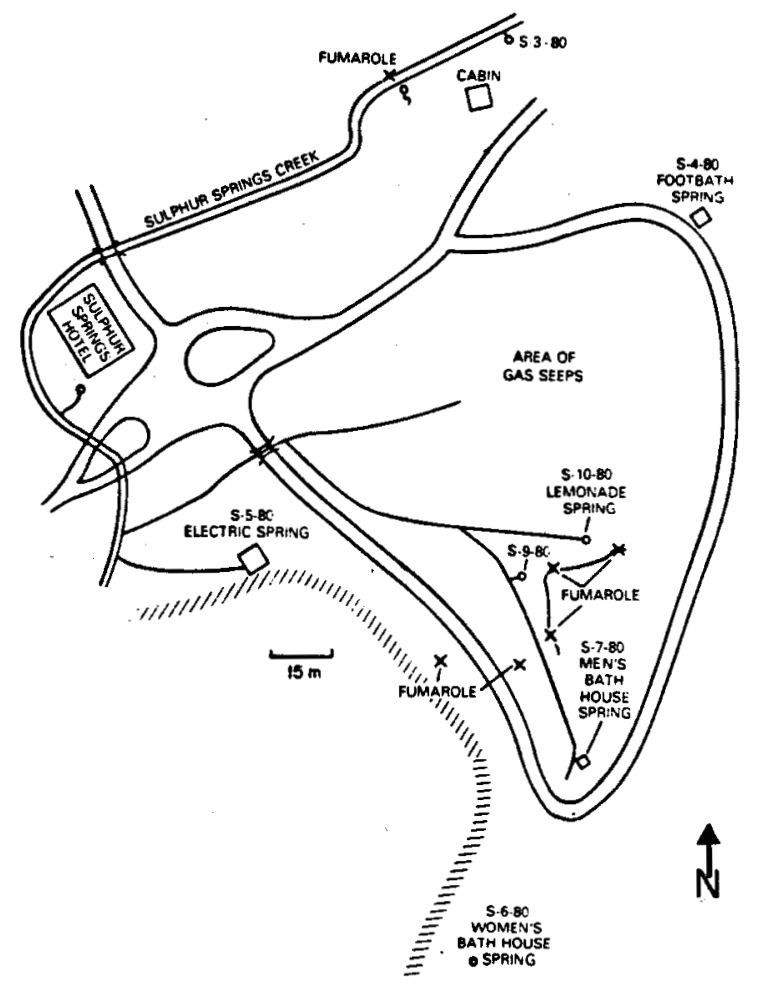

Fig. 3.

Sketch map of Sulphur Springs area (modified from Summers ${ }^{24}$ ).

different settings produce subtle differences in the chemistry of near-surface waters.

The hydrology of the Pajarito Plateau has been described by Purtymun and Johansen ${ }^{8}$ and the geochemistry of the waters with respect to geothermal potential has been discussed in detail by Goff and Sayer. ${ }^{3}$ Hydrology of the southern Jemez Mountains is not known in detail although the Jemez River and tributaries drain Valles Caldera in a southerly direction.

In general, surface and nearsurface waters of this category are

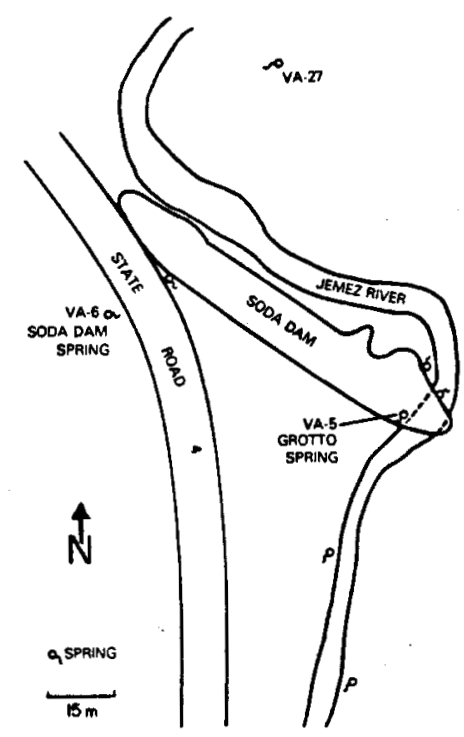

Fig. 4.

Sketch map of Soda Dam Area.

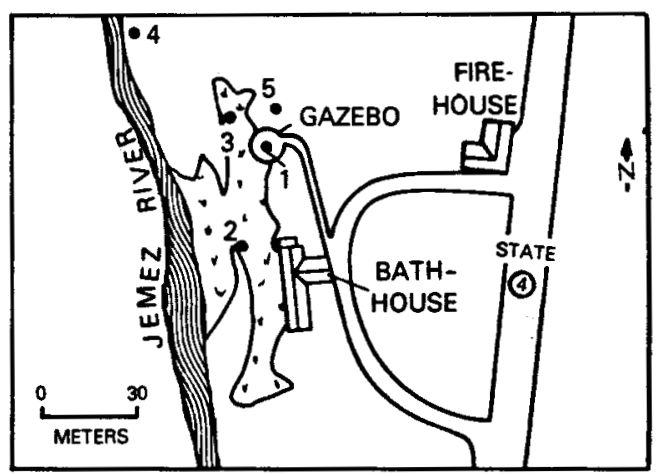

1. MAIN JEMEZ SPRING, VA-10

2. TRAVERTINE MOUND SPRING, VA-7

3. UNNAMED WARM SPRING, VA-12

4. BUDDHIST SPRING, VA-8

5. JEMEZ SPRINGS GEOTHERMAL WELL, VA-19

Fig. 5 .

Sketch map of Jemez Springs are (modified from Summers ${ }^{24}$ ). 


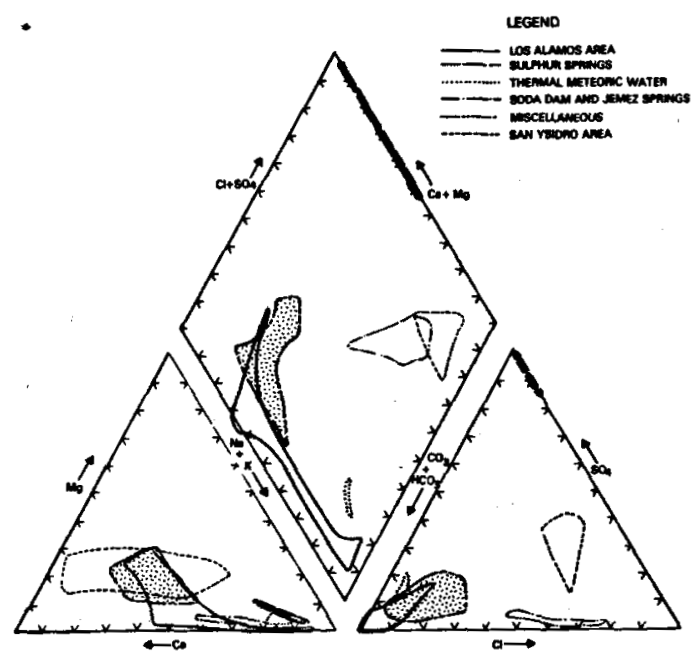

Fig. 6.

Piper diagram showing the ranges in chemical composition in equivalents of various types of water in southern Jemez Mountains, New Mexico. Patterns are added to help distinguish overlapping fields of data points.

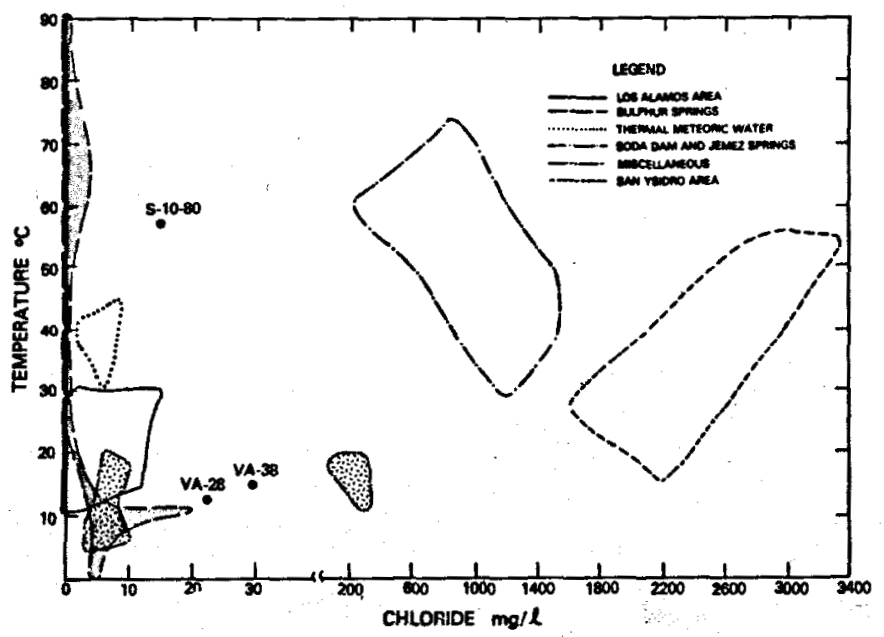

Fig. 8.

Plot of $\mathrm{Cl}$ vs measured temperature for various types of water in southern Jemez Mountains region, New Mexico. Sample numbers refer to waters that fall outside fields of generalized water types. Note that miscellaneous meteoric waters fall into two distinct fields. Those with high $\mathrm{Cl}$ issue from Paleozoic-Mesozoic rocks.

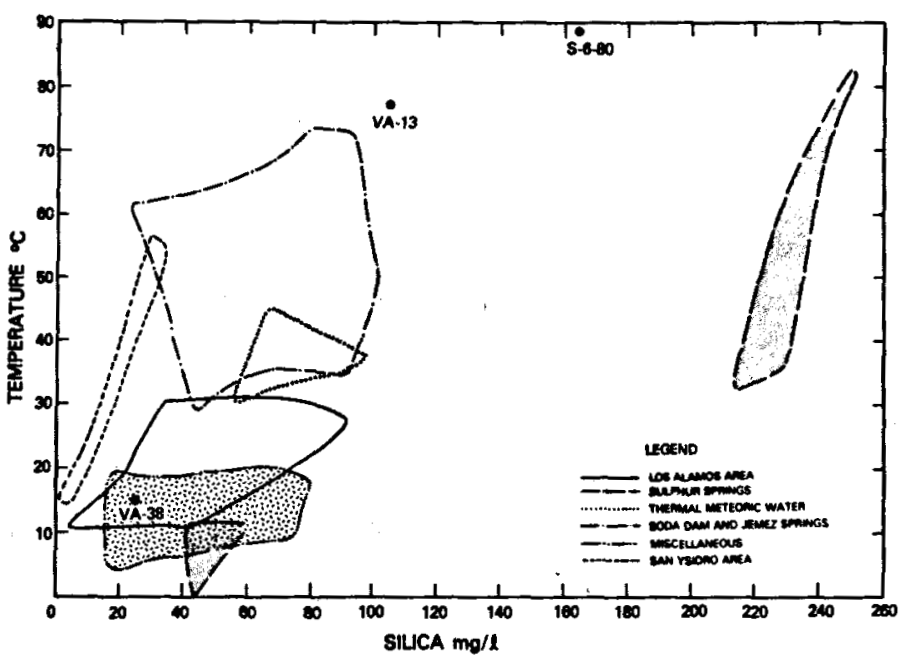

Fig. 7.

Plot of $\mathrm{SiO}_{2}$ vs measured temperature for various types of water in southern Jemez Mountains region, New Mexico. Sample numbers refer to waters that fall outside fields of generalized water types. Note that waters from Sulphur Springs area fall into two distinct fields.

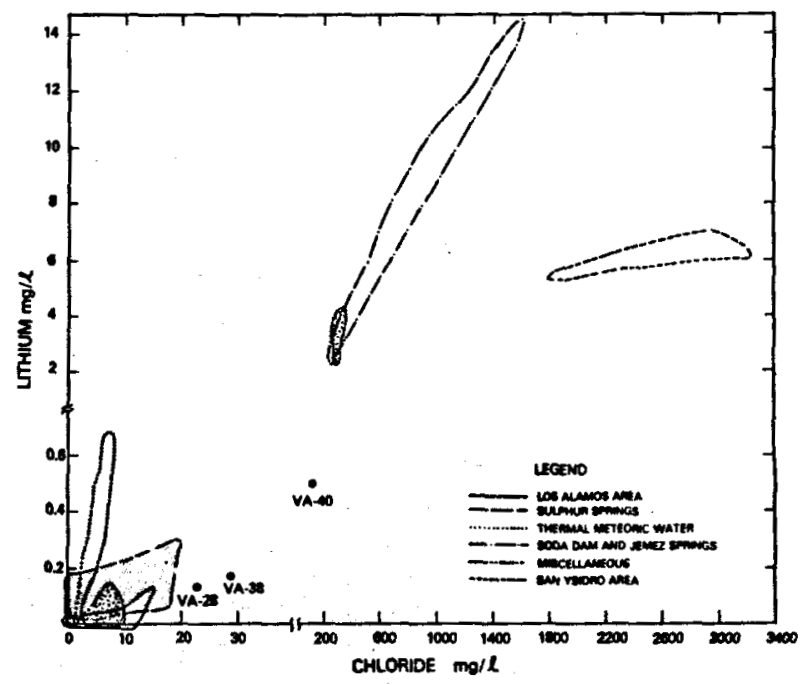

Fig. 9.

Plot of $\mathrm{Li}$ vs $\mathrm{Cl}$ for various types of water in southern Jemez Mountains region, New Mexico. Sample numbers refer to waters that fall outside fields of generalized water types. Note that miscellaneous meteoric waters fall into two distinct fields. Those with high $\mathrm{Li}$ issue from Paleozoic-Mesozoic rocks. 


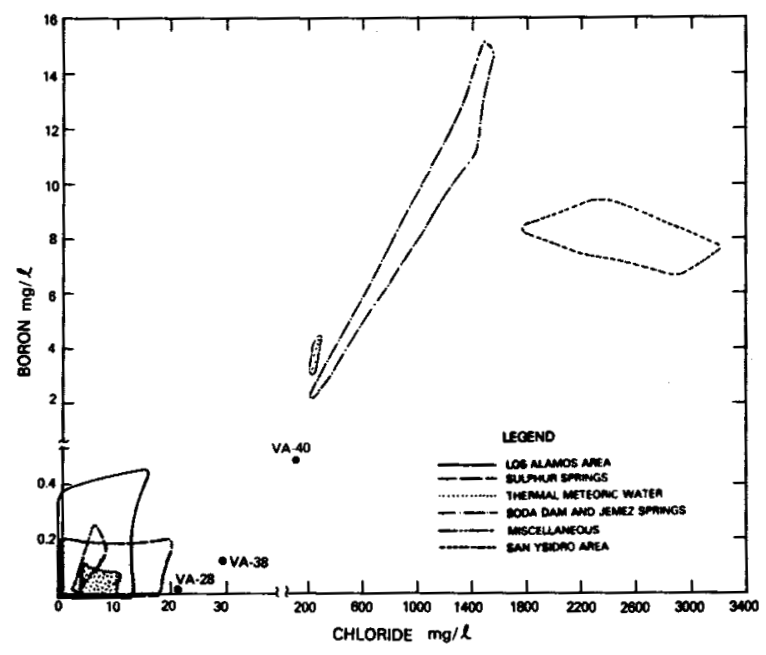

Fig. 10.

Plot of $B$ vs Cl for various types of water in southern Jemez Mountains region, New Mexico. Sample numbers refer to waters that fall outside fields of generalized water types. Note that miscellaneous meteoric waters fall into two distinct fields. Those with high $B$ issue from Paleozoic-Mesozoic rocks.

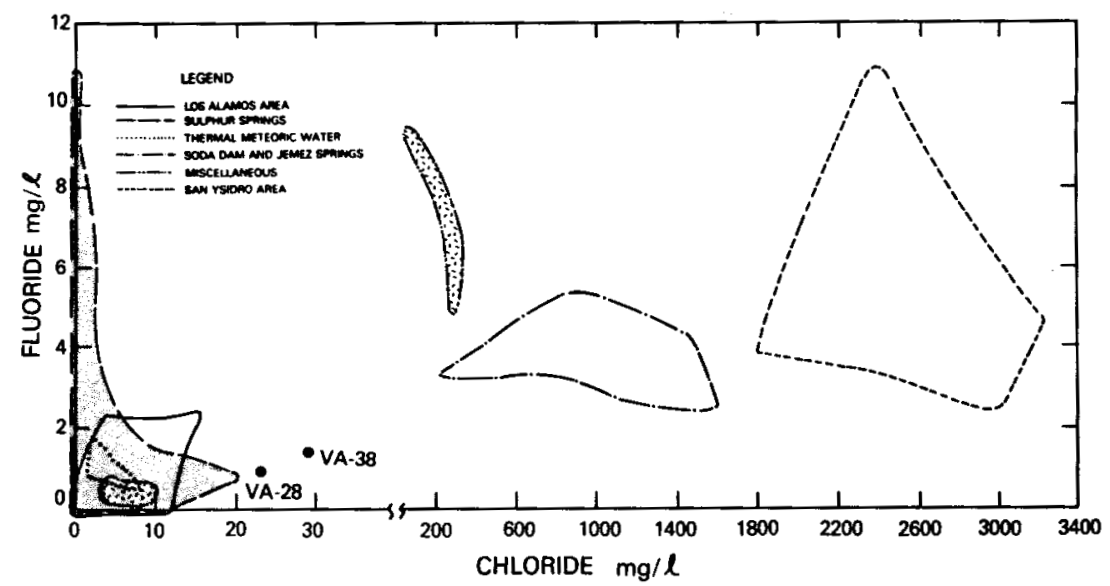

Fig. 11.

Plot of $\mathrm{F}$ vs $\mathrm{Cl}$ for various types of water in southern Jemez Mountains region, New Mexico. Sample numbers refer to waters that fall outside fields of generalized water types. Note that miscellaneous meteoric waters fall into two distinct fields. Those with high $F$ issue from Paleozoic-Mesozoic rocks. 


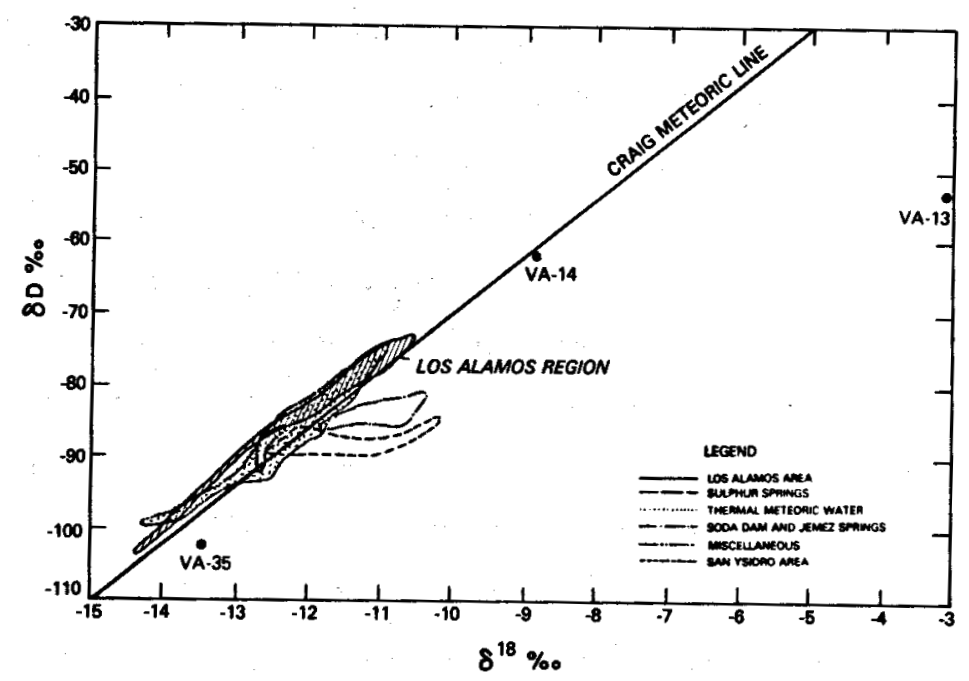

Plot of $\delta D$ vs $\delta^{18} 0$ for various types of water in southern Jemez Mountains region, New Mexico. Sample numbers refer to waters that fall outside fields of generalized water types. Two hot springs from Sulphur Springs (VA-13, VA-14) are plotted separately.

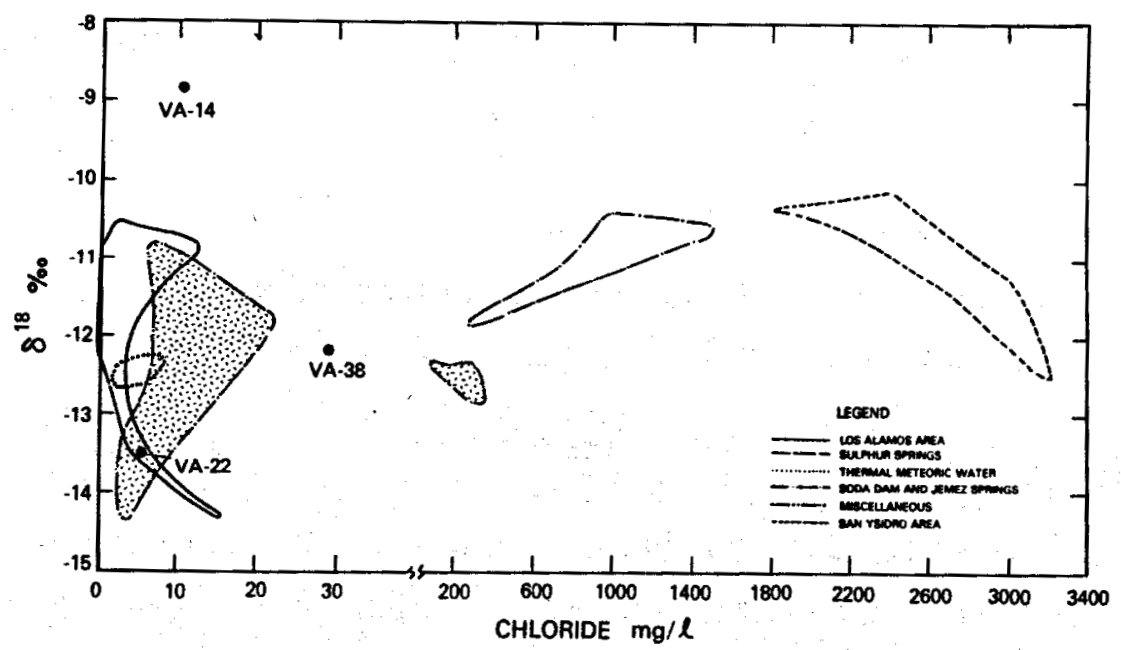

Plot of $\delta^{18} 0$ vs $\mathrm{Cl}$ for various types of water in southern Jemez Mountains region, New Mexico. Sample numbers refer to waters that fall outside fields of generalized water types. One hot spring from Sulphur Springs (VA-14) is plotted separately. Note that miscellaneous meteoric waters fall into two distinct fields. Those with high $\mathrm{Cl}$ issue from Paleozoic-Mesozoic rocks. 
calcium-bicarbonate waters although some of the Los Alamos water-supply wells and cold mineral springs in the Valles Caldera region are sodium-bicarbonate waters. The waters can be characterized by low conductivity (since they are relatively dilute), low temperature and $\mathrm{pH}$ near 7 . They typically contain low concentrations of $\mathrm{F}, \mathrm{Cl}, \mathrm{Li}, \mathrm{B}$, and $\mathrm{SiO}_{2}$, (Figs. 7, 8, 9, 10, and 11) indicating a low temperature, near-surface environment. ${ }^{22}$ The Los Alamos waters show a wider range in all these constituents than most cold waters of the region, possibly due to long residence time of some waters in deep aquifers or to some mixing with deep thermal/mineral water rising along faults in the Pajarito Plateau. ${ }^{3}$ A few springs in the Caldera region are anomalous in that they are somewhat mineralized but cold (location nos. 34, 36, 37, and to a lesser extent location 31, Fig 2; samples VA-31, VA-39, VA-40, and VA-28, Table B-II). These samples have much greater conductivity, comparatively high $\mathrm{Na}+\mathrm{K}$ relative to $\mathrm{Ca}$, high $\mathrm{Cl}$ relative to $\mathrm{HCO}_{3}$, and relatively high $\mathrm{F}$, $\mathrm{B}$, and Li (see Figs. 8, 9, 10, 11, and 13). Two of the springs (location nos. 34 and 36) al so have very low $\mathrm{SiO}_{2}$. These slightly mineralized meteoric waters issue from Mesozoic-Paleozoic sediments and either leach out evaporitic minerals from them, or else contain an extremely small fraction of deep thermal water from Valles Caldera.

Trace elements of all waters in this group are fairly low (Table B-III). $\mathrm{Sr}$ appears to be higher in waters issuing from Madera limestone (especially location nos. 34 and 36 ), suggesting that $\mathrm{Sr}$ is dissolved out of the carbonate rocks.

The $D$ and ${ }^{18} 0$ isotope ratios follow Craig's ${ }^{23}$ line for meteoric waters (Fig. 12). The cold mineral waters described above are not isotopically distinct from other waters in this group.

\section{B. Valles Caldera-Sulphur Springs Area}

A suite of thermal and nonthermal acid-sulfate springs and mudpots and some associated dilute carbonated waters are located within the Valles Caldera near the western margin of the resurgent dome (Figs. 2 and 3 ). Discussion of the chemistry and origin of the Sulphur Springs has been presented by Goff and Grigsby ${ }^{1}$ and Trainer. ${ }^{5}$ Names used for various springs in Tables B-I, B-II, B-III, and B-IV correspond with those presented by Summers. ${ }^{24}$ Rhyolitic flows and tuffs and caldera fill deposits in the area have been extensively altered and leached to clays, silica minerals, authigenic feldspars and sulfates. 
Native sulfur, pyrite and aluminum sulfates have precipitated near fumaroles (R. Charles and D. Bish, Los Alamos National Laboratory, personal communication, 1981). The waters are characterized by a high conductivity (except for the dilute bubbling waters), and low pH, especially in the most concentrated waters. Most of the samples have moderate $\mathrm{F}$ and low B, Li, and $\mathrm{Cl}$ (Figs. 8, 9, 10, and 11). The $F$ concentrations are nighest in the $\mathrm{Cl}$-free waters. $\mathrm{SiO}_{2}$ contents vary within the suite but the waters can generally be divided into two groups: a high-temperature, high-SiO 2 group and a low-temperature, low-SiO 2 group (Fig. 7). This relationship is in part a function of the increasing solubility of $\mathrm{SiO}_{2}$ with increasing temperature.

Trace elements $\mathrm{Zn}, \mathrm{Cu}, \mathrm{Cr}, \mathrm{Co}$, and $\mathrm{Ni}$ show relatively high concentrations in acid waters that are also rich in Fe (Table B-III), a relationship probably due to the greater solubilities of these metals in acid waters.

The $D$ and ${ }^{18} 0$ isotope ratios of the bubbling seep (sample VA-23; location no. 22, Fig. 2) and unnamed acid spring (sample VA-14; location no. 21, Fig. 2) both fall on Craig's meteoric line, the unnamed spring being more enriched in ${ }^{18_{0}}$ and $D$ (Fig. 12). In contrast, the Men's Bathhouse mudpot (sample VA-13) consists mostly of condensed steam from depth and is very enriched in ${ }^{18} 0$, probably the result of extensive hot water rock interactions at depth.

The Sulphur Springs are typical of a vapor-dominated geothermal system ${ }^{25}$ where water vapor, $\mathrm{H}_{2} \mathrm{~S}$, and $\mathrm{CO}_{2}$ rise from an underlying boiling water table. The known occurrence of a high temperature $\left(260^{\circ} \mathrm{C}\right)$ hydrothermal system beneath the resurgent dome of Valles Caldera ${ }^{20}$ indicates that such a boiling water table may exist beneath Sulphur Springs. Condensation of the steam near the surface and surface oxidation of $\mathrm{H}_{2} \mathrm{~S}$ to $\mathrm{H}_{2} \mathrm{SO}_{4}$ results in acid-sulfate water. The flowing springs mix seasonally with surface meteoric water since they show lower flow rates and higher temperatures during the dry months of the year.

\section{Valles Caldera - Thermal Meteoric}

Included here are a group of warm springs in the western ring fracture zone, previously described by Goff and Grigsby, ${ }^{1}$ Goff and Sayer, ${ }^{3}$ and Trainer. 5 The waters are typically dilute, near neutral in $\mathrm{pH}$ and have moderate temperature $\left(31-45^{\circ} \mathrm{C}\right)$. They are sodium-bicarbonate waters displaying low concentrations of $\mathrm{F}, \mathrm{Cl}, \mathrm{B}$, and $\mathrm{Li}$ (Figs. 8, 9, 10, and 11). The F, B, and $L i$ contents are slightly higher than most of the surface waters in the Caldera and $B$ and $L i$ concentrations are both slightly enriched in samples with 
nigher $\mathrm{Cl}$. McCauley Spring differs from the others in that it contains more $\mathrm{Ca}$ and $\mathrm{Mg}$ and less alkalis. All samples show low concentrations of the trace elements analyzed (Table B-III). The $D$ and ${ }^{18} 0$ isotopes fall close to Craig's meteoric line in a tight cluster (Fig. 12).

These waters can be considered to be meteoric waters that have been heated during circulation in the ring fracture zone of the Valles caldera. All waters of this type discharge near the youngest (less than $0.5 \mathrm{Myr}$ ) of the moat rhyolites erupted in the Caldera. They are slightly depleted in Ca and $\mathrm{Mg}$ compared with surface waters in the Caldera region due to the inverse relationship between carbonate solubility and temperature. Very slight mixing with deep thermal waters may have occurred in Spence, Little Spence, and possibly, McCauley Hot Springs (location nos. 25 and 26, Fig. 2; samples VA-1, $V A-2$, and $V A-3$ ) because they have higher $\mathrm{Li}$ and $\mathrm{Na}$ than most of the meteoric waters in the Valles Caldera region.

D. Valles Caldera - Deep Thermal Waters and Derivatives

A number of hot springs and warm springs occur along the trace of the Jemez fault zone in Cañon de San Diego, including springs at Soda Dam (Fig. 4) and near the village of Jemez Springs (Fig. 5). The Jemez fault zone extends southwest and south from the Caldera for about $20 \mathrm{~km}$. It was active before eruption of the Jemez Mountains volcanic field ${ }^{2}$ and locally represents the western boundary of the Rio Grande rift. The hot springs occur at intersections of the Jemez River with the fault and fracture zones. Chemical composition and origin of these mineralized springs has been discussed by Goff et al., ${ }^{2}$ Trainer, ${ }^{5}$ and in part by Goff and Grigsby ${ }^{1}$ and Goff and Sayer. ${ }^{3}$ Soda Dam, Travertine Mound Spring, and some others are actively depositing carbonate travertine.

The springs display fairly high conductivity and are neutral to slightly acid sodium-chloride to calcium-bicarbonate waters. The $\mathrm{Cl}$ and $\mathrm{Ca}$ concentrations show a slight inverse relationship suggesting a mixing of deep thermal water and surface meteoric water. They contain moderate $F$ and high $L i$ and $B$ concentrations with a marked trend of increasing $B$ and $L i$ with increasing $C l$ (Figs. 9, 10, and 11).

$\mathrm{Sr}$ is the most noteworthy trace element in these waters, ranging from 0.4 to $2 \mathrm{mg} / \mathrm{l}$ (Table B-III). There is an approximate trend of increasing $\mathrm{Sr}$ with increasing $\mathrm{Ca}$. The waters have probably derived most of their $\mathrm{Ca}$ and $\mathrm{HCO}_{3}$ by 
flow through fractured Paleozoic carbonate rocks and this is probably the main source of $\mathrm{Sr}$.

The $D$ and ${ }^{18} 0$ isotopes fall away from the meteoric line of Craig, ${ }^{23}$ showing a distinct trend of ${ }^{18} 0$ enrichment (Fig. 12). Water from Travertine Mound Spring and from the well near main Jemez Spring are isotopically most similar to meteoric water and the main Jemez and Soda Dam Springs show the most relative enrichment in ${ }^{18} 0$.

These thermal waters are probably derivatives of the deep geothermal fluids beneath Valles Caldera, which are almost pure $\mathrm{NaCl}$ waters. 2 Mixing of this hot $\mathrm{NaCl}$ water with calcium-bicarbonate meteoric water has cooled it down and diluted it. This mixed water dissolves $\mathrm{CaCO}_{3}$ while flowing southwest through shattered Paleozoic sediments along the Jemez fault zone, giving rise to the higher $\mathrm{Ca}$ and $\mathrm{HCO}_{3}$ contents observed in the springs. This mixing is supported by oxygen isotopes since the deep geothermal water from valles Caldera is much more enriched in ${ }^{18} 0$ relative to meteoric water. ${ }^{1}$

\section{E. San Ysidro Area}

A number of warm springs and hot and cold water wells including mineral and nonmineral waters were sampled in the San Ysidro-Jemez Pueblo area. Many of the samples were collected for isotope analysis up to two years before they were collected for chemical analysis. The chemistry of the waters in this area has been discussed by Goff et al., ${ }^{2}$ Goff and Sayer, ${ }^{3}$ Mariner et al., 26 and Trainer. 5,6

The waters are low- to moderate-temperature, near-neutral, concentrated (except for dilute surface water) sodium-chloride to sodium-sulfate waters. They contain much more $\mathrm{SO}_{4}, \mathrm{Na}$, and $\mathrm{Cl}$ than the thermal waters discharging along the Jemez fault zone (Table B-II) except for Ow1 Spring, which resembles the surface meteoric calcium-bicarbonate water. The concentrated waters have very high $\mathrm{Cl}$ and moderate to high $\mathrm{F}, \mathrm{B}$, and $\mathrm{Li}$ contents (Figs, 8, 9, 10, and 11). Silica contents are much lower than most other waters described in this report although concentrations of silica increase with temperature (Fig. 7).

Trace element concentrations are low except for $\mathrm{Sr}$, which increases with Ca concentration (Table B-III). Sr contents are much higher than waters from the Jemez fault zone with comparable $\mathrm{Ca}$.

Most of the $\mathrm{SO}_{4}$ in these waters may be derived from massive gypsum deposits (Jurassic Todilto Formation) near San Ysidro. Most of the springs 
issue from pre-Jurassic sediments so the $\mathrm{SO}_{4}$ is probably derived from both pore fluids in the rocks and from downward percolating ground water. Assuming $\mathrm{Ca}$ is also derived in part from gypsum, the higher $\mathrm{Sr} / \mathrm{Ca}$ in these waters compared with the Jemez and Soda Dam Springs may result from greater leaching of $\mathrm{Sr}$ from the gypsum than from carbonate.

Several chemical features make these waters distinct from the waters derived from the deep geothermal system in the Valles Caldera. ${ }^{2}$ The ratios of $\mathrm{Na} / \mathrm{Cl}$ and $\mathrm{B} / \mathrm{Cl}$ in the Jemez Springs waters are 1.3-1.9 and 0.008-0.009, respectively, whereas the same ratios in the San Ysidro waters are 0.8-1.2 and 0.002-0.005, respectively. Li/Na ratios in waters from the Jemez Springs area are in the range 0.012-0.019 compared with 0.002-0.005 for waters from the San Ysidro region. Finally the isotopic enrichment of ${ }^{18} 0$ in San Ysidro waters follows a parallel but different trend (Fig. 12) than deep Caldera waters. All these data indicate that the San Ysidro waters originate from a separate geothermal system near the Nacimiento fault zone rather than the one located beneath Valles Caldera.

\section{SUMMARY}

The geologic, chemical, and isotopic data presented here are sufficient to characterize the different thermal and nonthermal waters of the Valles Caldera-southern Jemez Mountains region. Further interpretations are beyond the scope of this report. However, the authors feel this data can be applied to the solution of other hydrologic and geochemical problems of the region. Perhaps, the most interesting problem would be to determine the age, geochemical evolution, and hydrologic balance of the high-temperature geothermal system within Valles Caldera.

\section{ACKNOWLEDGMENTS}

Many people at Los Alamos have contributed to the collection and analysis of waters listed in this report: Ron Aguilar, Kevin Ferdinand, Jamie Gardner, Kim Kariya, Tino Lucero, Dave Mann, Suzanne Sayer, Bruce Stewart, and Rosemary Vidale. L. Merlivatt, Departement de Recherche et Analyse, Saclay, France, provided the isotope analyses. J. Husler, University of New Mexico, analyzed major elements for samples VA-1 to VA-6 and LA-1 to LA-20. The previous work of Frank Trainer and Bill Purtymun is gratefully acknowledged. 
UNUSUAL SPRINGS OF VALLES CALDERA AND SOUTHERN JEMEZ MOUNTAINS, NEW MEXICO

I. SULPHUR SPRINGS, VALLES CALDERA

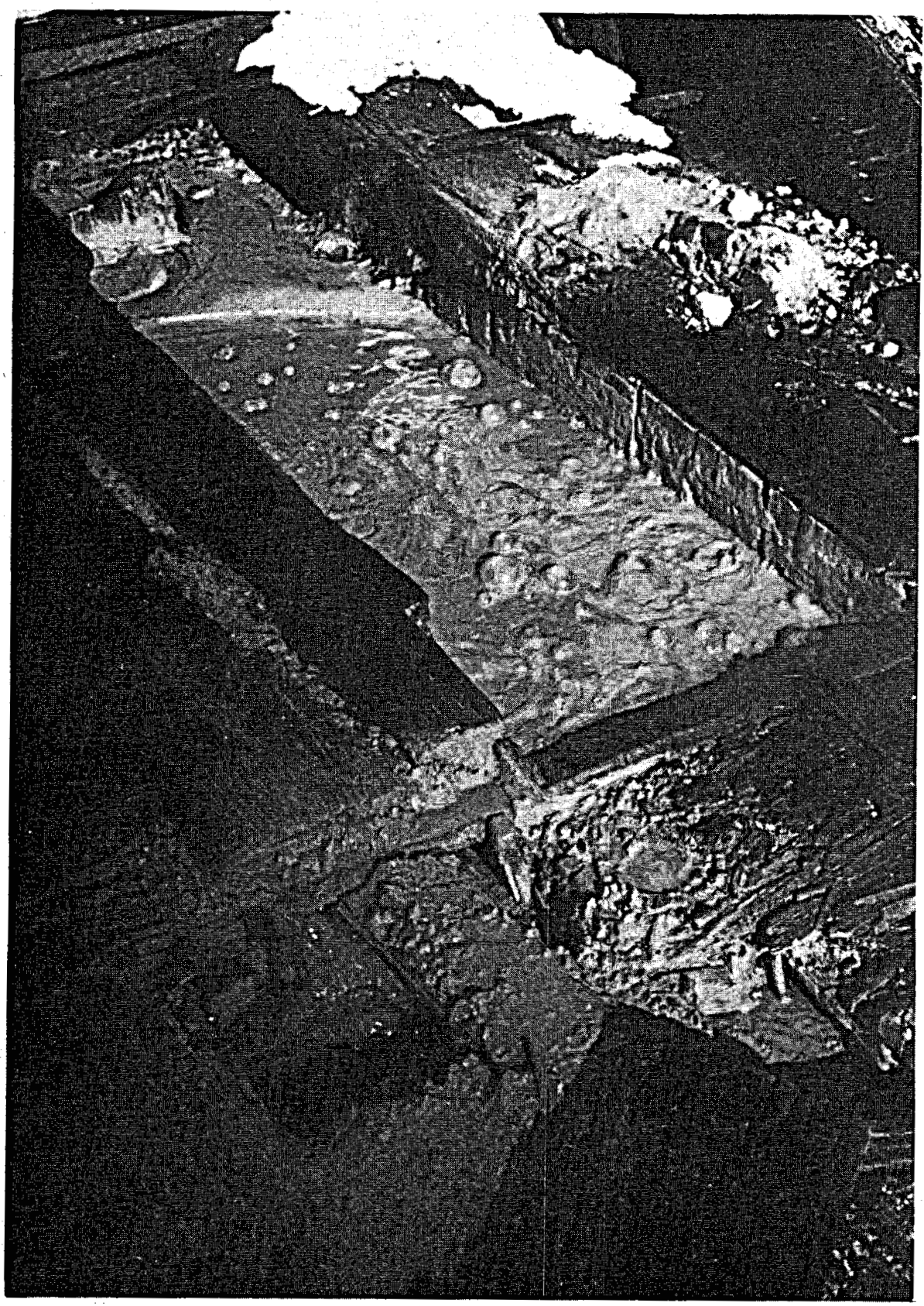

Fig. A-1.

Men's Bathhouse Mudpot in January $1979 ; \mathrm{pH} \approx 2.0$, temperature $\approx 80^{\circ} \mathrm{C}$. The bathhouse that is now collapsed once held several concrete pools of acidsulfate muddy water at various temperatures suitable for bathing. The mudpot occurs on a small knoll from which several fumaroles, $93^{\circ} \mathrm{C}$, discharge sulfurous gases. 


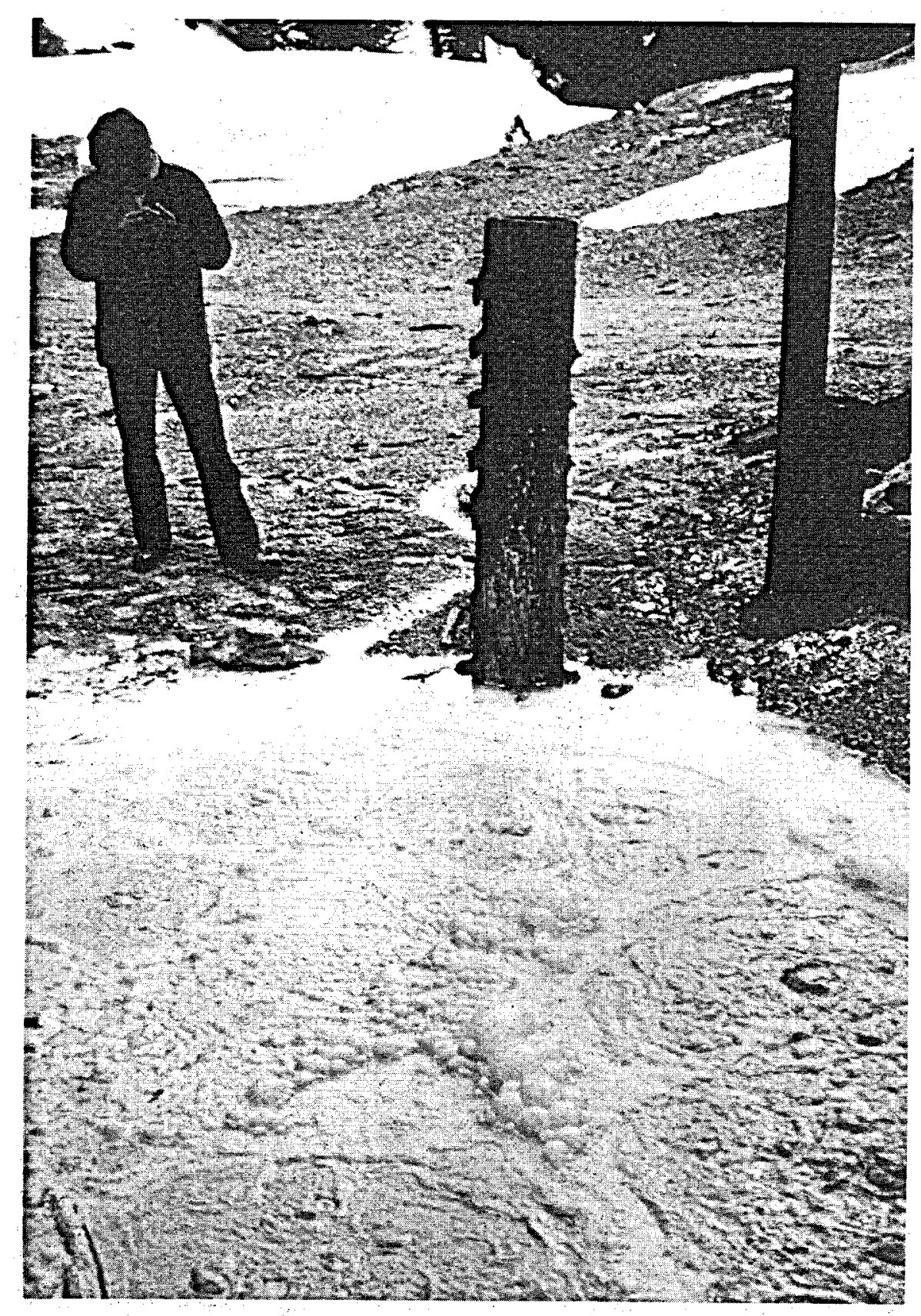

Fig. A-2.

Footbath Spring in March $1980 ; \mathrm{pH} \cong 1.0$, temperature highly variable. The pale yellow color of the suspended mud is due to colloidal sulfur. 


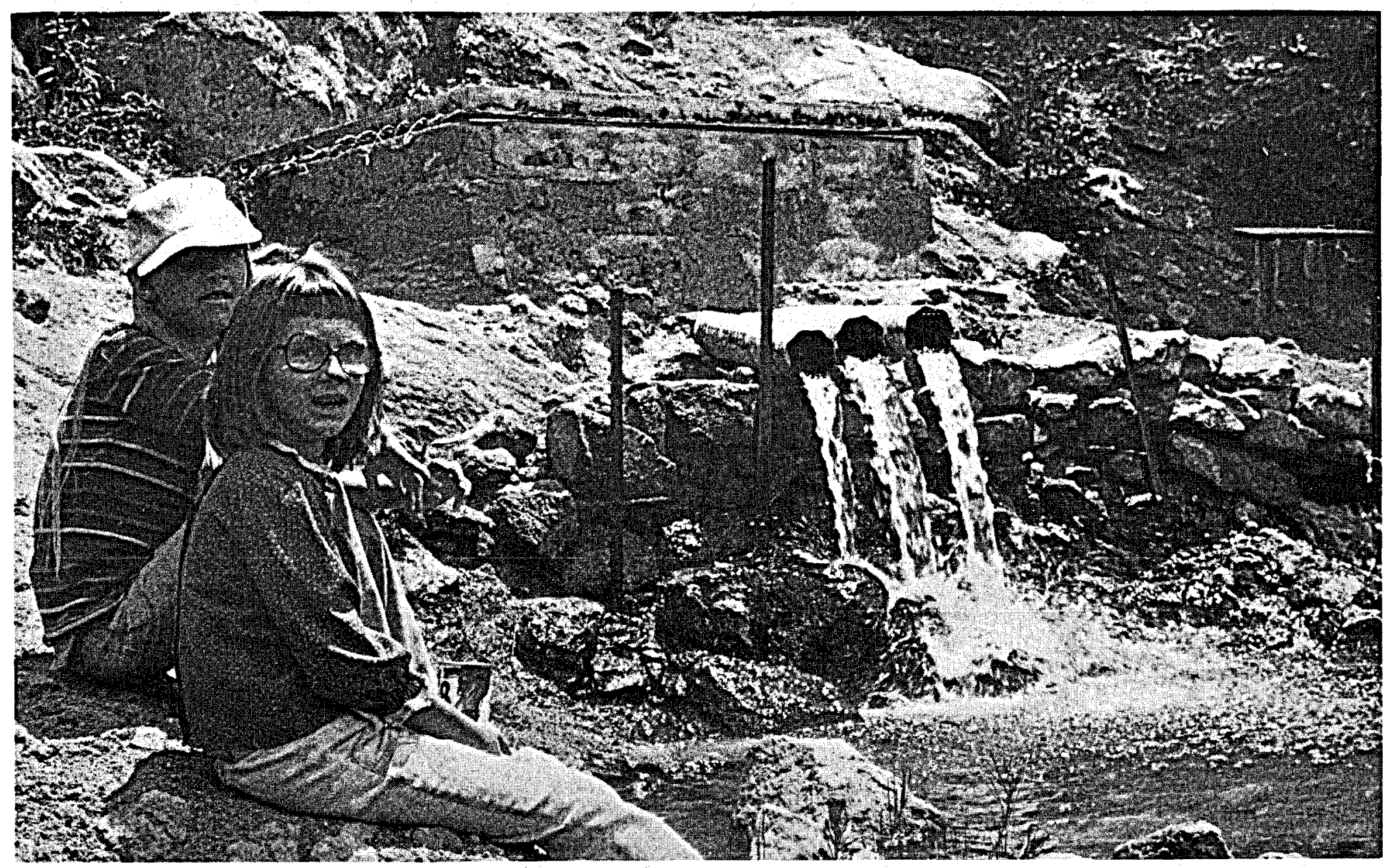

Fig. A-3

San Antonio Hot Spring in July 1978; $\mathrm{pH} \simeq 7$, temperature $=42^{\circ} \mathrm{C}$. Spring discharges from concrete crib on $\vec{\nabla}$ fractured rhyolite about $60 \mathrm{~m}$ above San Antonio Creek, a favorite destination of cross-country skiers. 


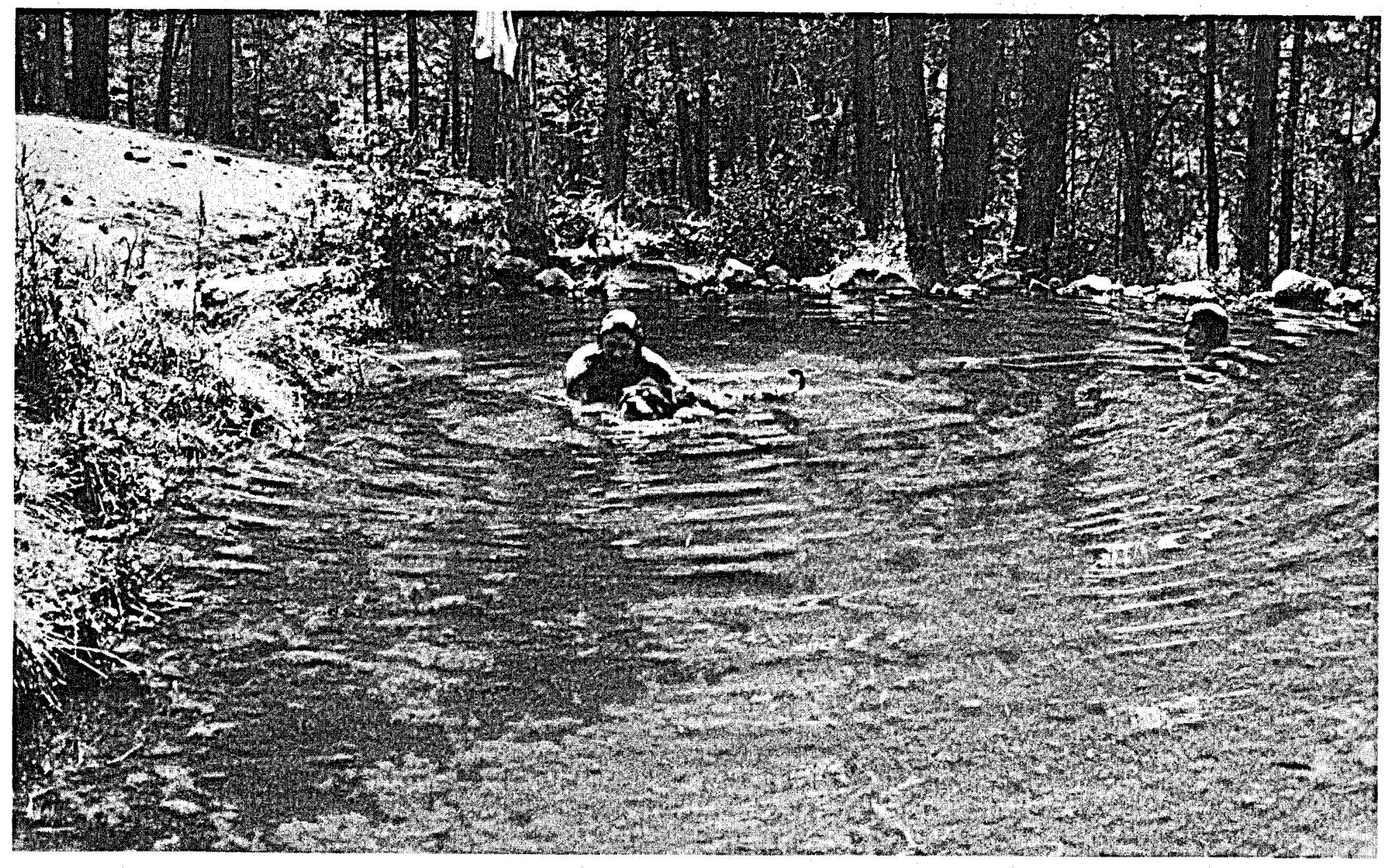

Fig. A-4.

McCauley Spring in July 1978; $\mathrm{pH} \simeq 6.5$, temperature $=31^{\circ} \mathrm{C}$. Visitors have built a rock swimming pool about $"$ $30 \mathrm{~m}$ wide and $1 \mathrm{~m}$ deep adjacent to the spring orifice. They have also stocked it with tropical freshwater fish. 
II1. JEMEZ FAULT ZONE, $10 \mathrm{~km}$. SOUTHWEST OF VALLES CALDERA

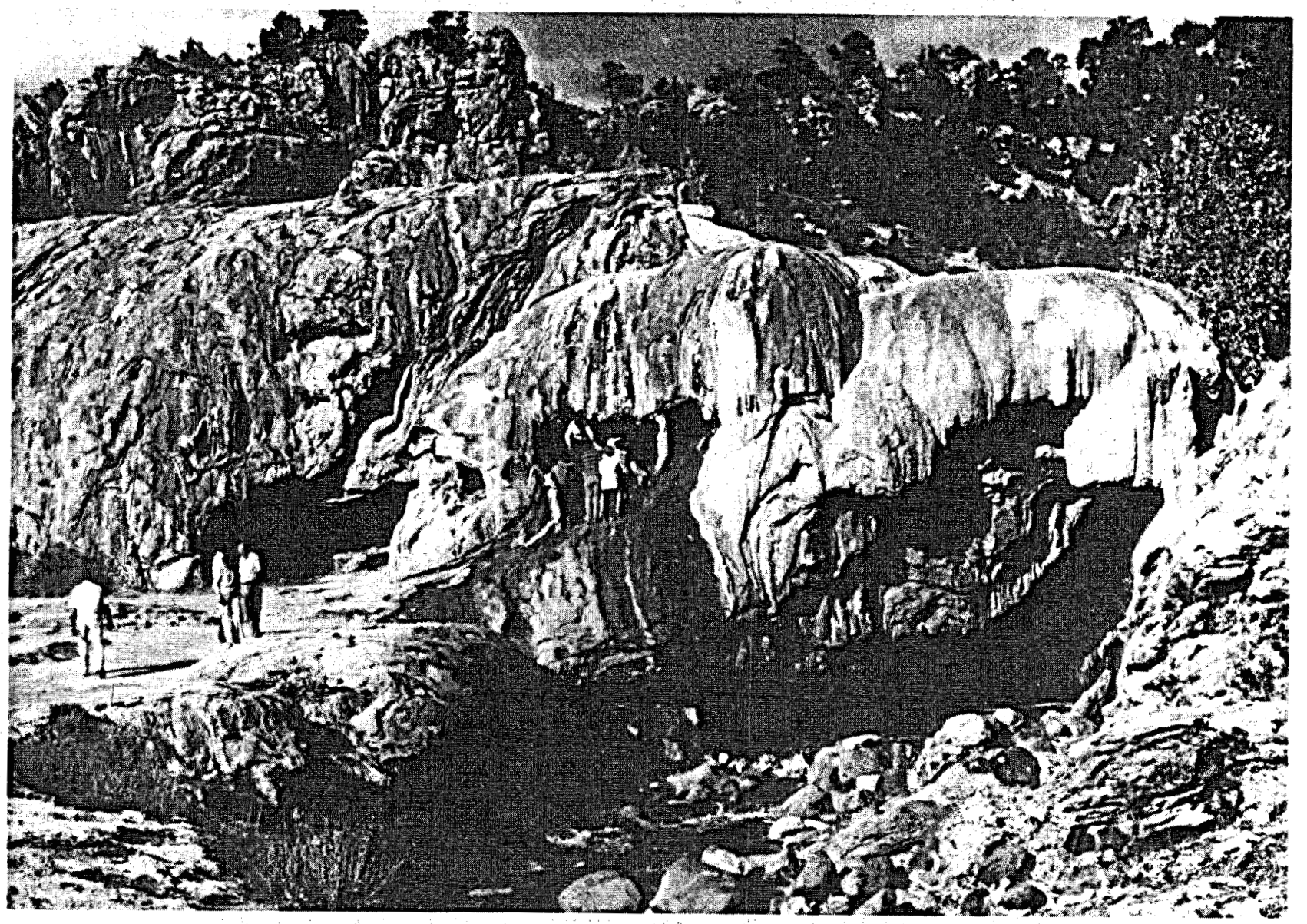

Fig. A-5.

Soda Dam looking north, April 1980. The dam is built of carbonate travertine deposited by bicarbonated hot springs, but construction of the present highway (just to left of photo) ruined the spring system. The existing dam is now disintegrating. Jemez River has undercut dam on right of photo. Sightseers are standing at the entrance to Grotto Spring. 


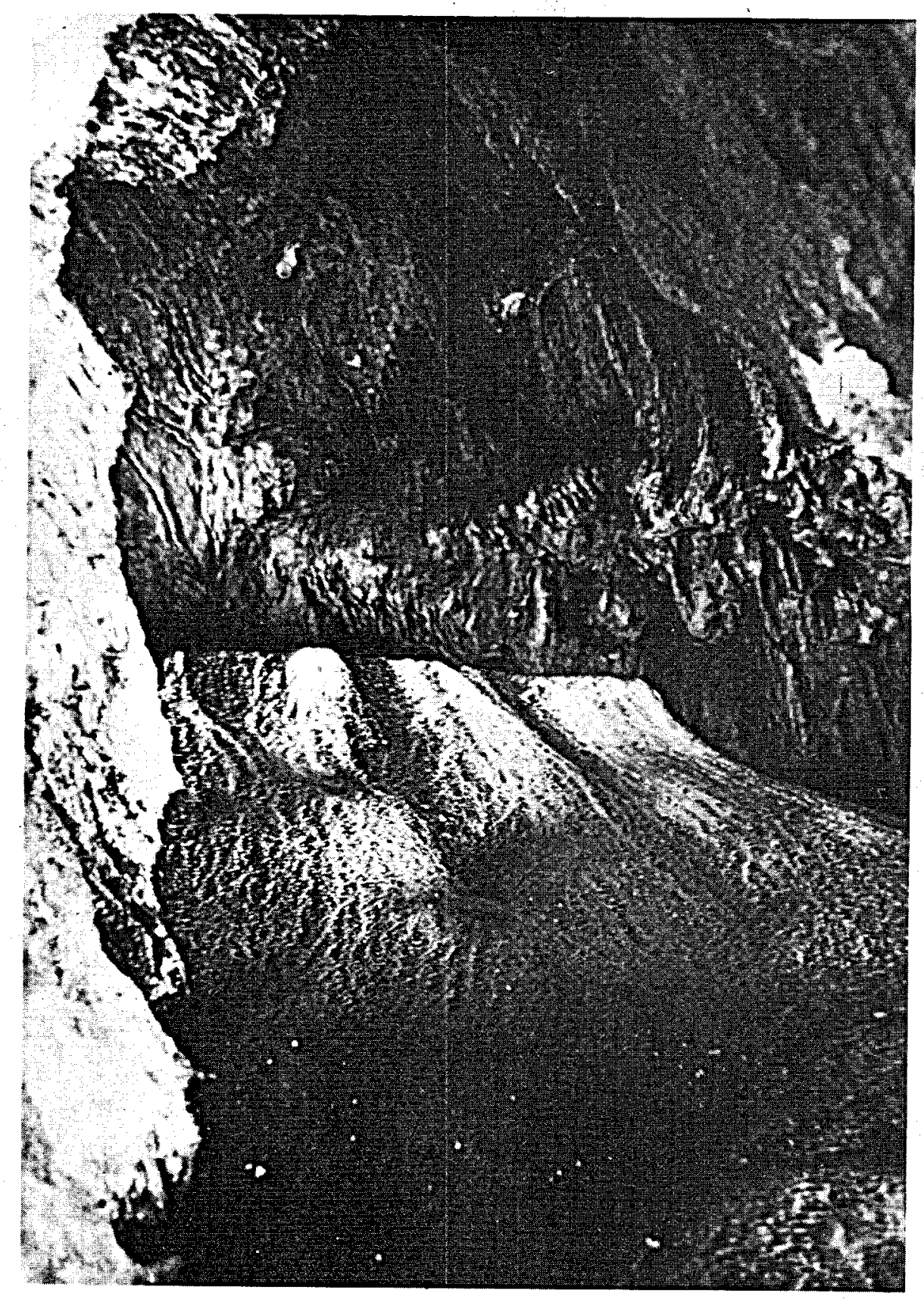

Fig. A-6.

Grotto Spring in October $1980 ; \mathrm{pH} \simeq 7$, temperature $=38^{\circ} \mathrm{C}$. This spring discharges from cave in Soda Dam shown on previous page. Although cooler in temperature, Grotto Spring has identical chemistry to Soda Dam Spring. 


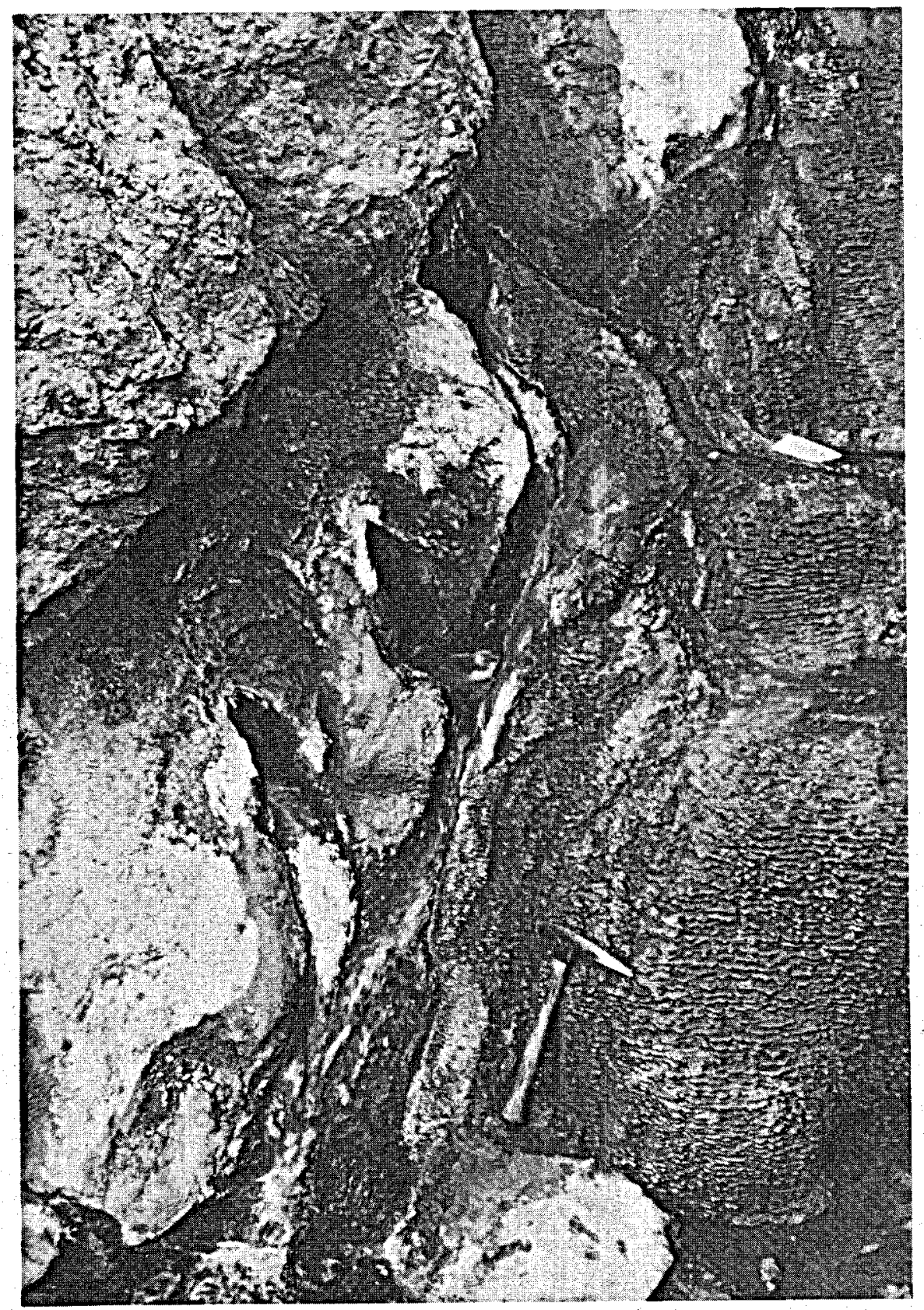

Fig. A-7.

Soda Dam Spring in October $1980 ; \mathrm{pH} \cong 6.3$, temperature $=47^{\circ} \mathrm{C}$. Spring issues from shear zone separating granite from vertically faulted sandstone along edge of State Highway 4. 


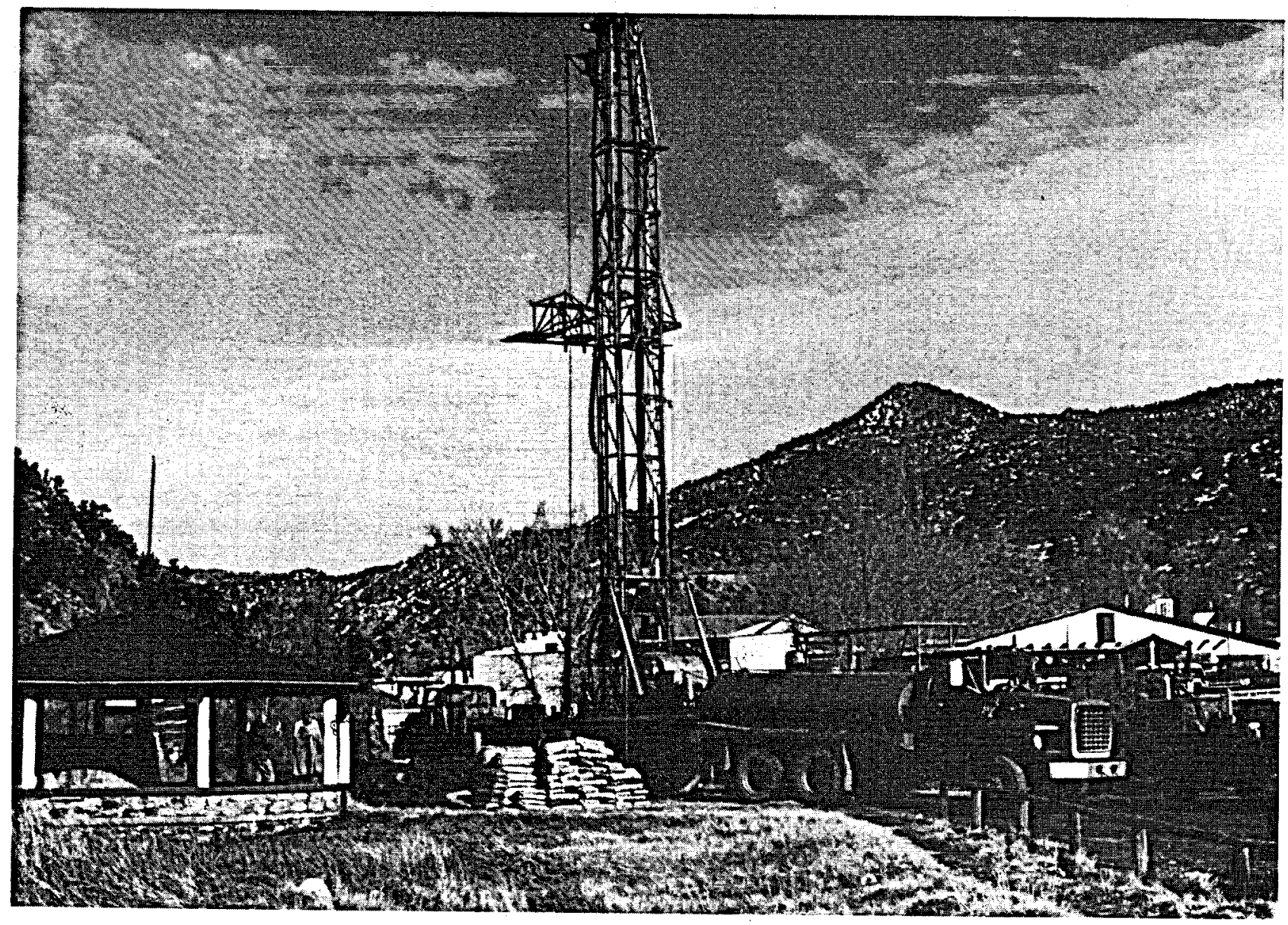

Fig. A-8.

Main Jemez Spring during drilling of Jemez Springs Geothermal Well, January, 1979. The gazebo covers the main spring, really an old shallow well, that supplies water to the bathhouse (behind photographer). The geothermal well struck hottest water, $72^{\circ} \mathrm{C}$, at $25-\mathrm{m}$ depth although total depth attained $255 \mathrm{~m}$. 


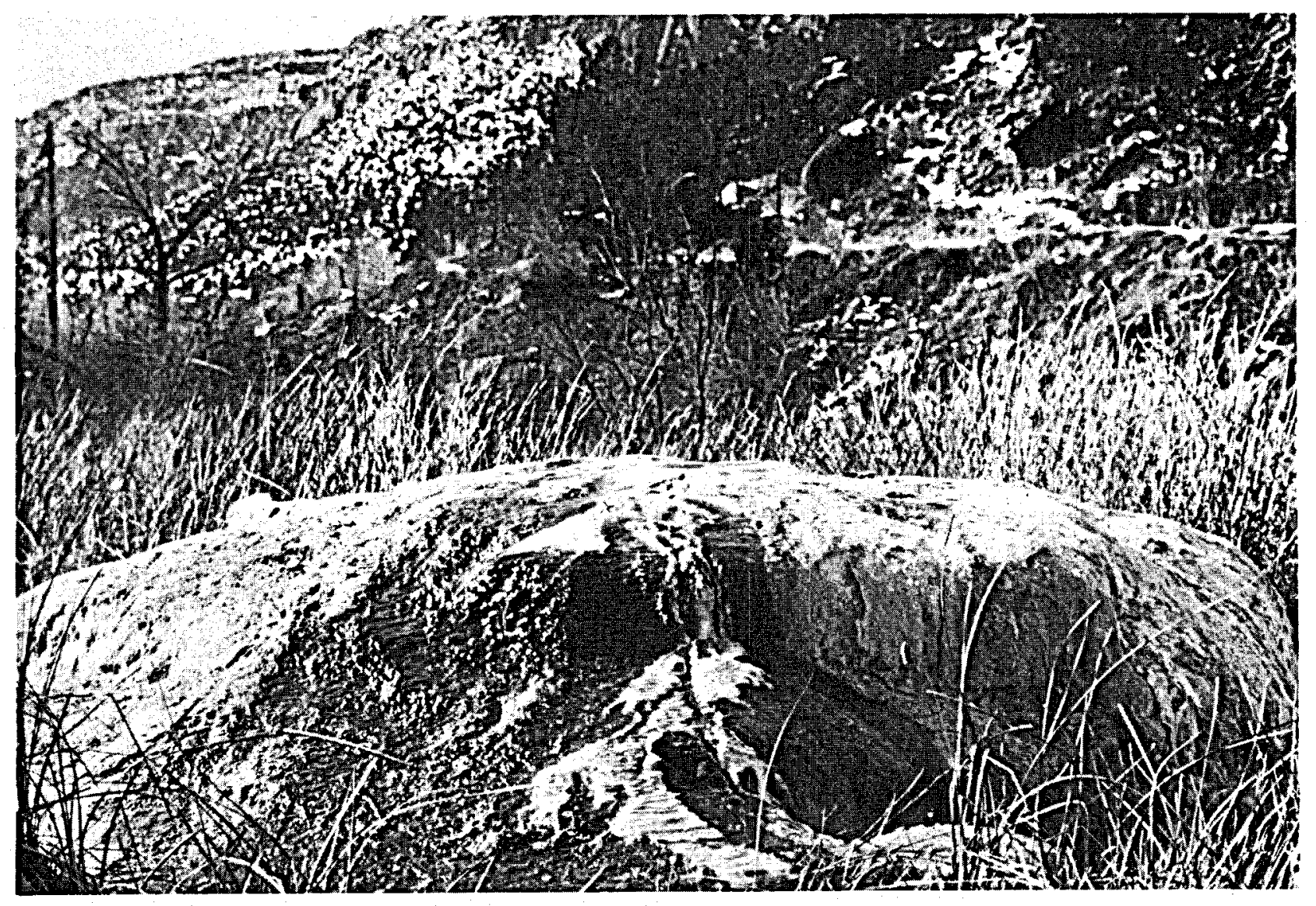

Fig. A-9.

Travertine Mound Spring in January 1979; $\mathrm{pH} \simeq 6.4$, temperature $=72 \dot{\mathrm{C}}$. This spring, which discharges between the bathhouse and Jemez River, contains an extremely rare species of algae. 
IV. SAN YSIDRO AREA

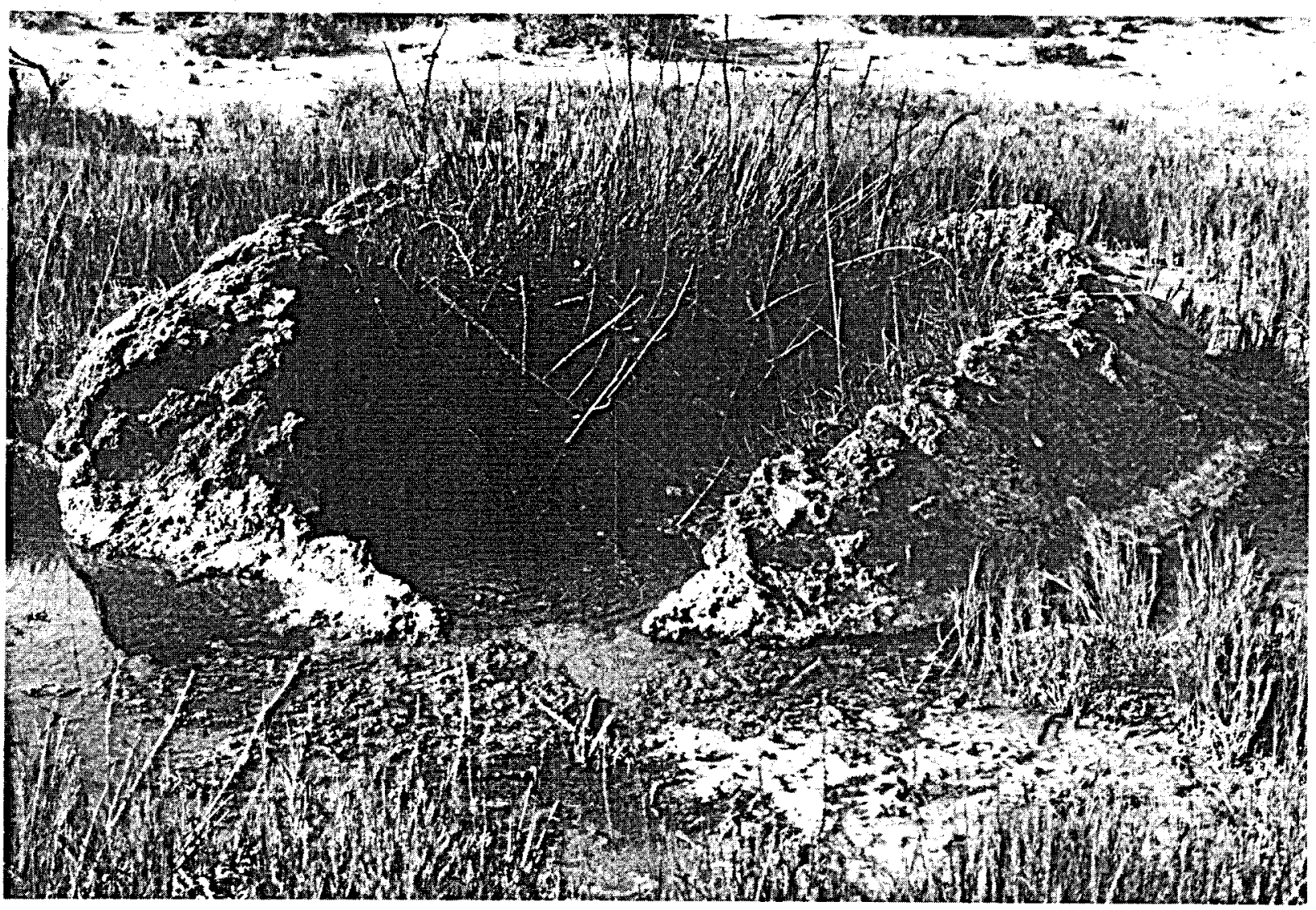

Fig. A-10.

Unnamed Mineral Spring in August 1979; $\mathrm{pH} \simeq 6.5$, temperature $\simeq 25^{\circ} \mathrm{C}$. Spring discharges about $20 \mathrm{~m}$ north of state highway and resembles 4 or 5 others within $200 \mathrm{~m}$. Water is rich in dissolved sulfate that is probably leached from gypsum-bearing rocks nearby. 


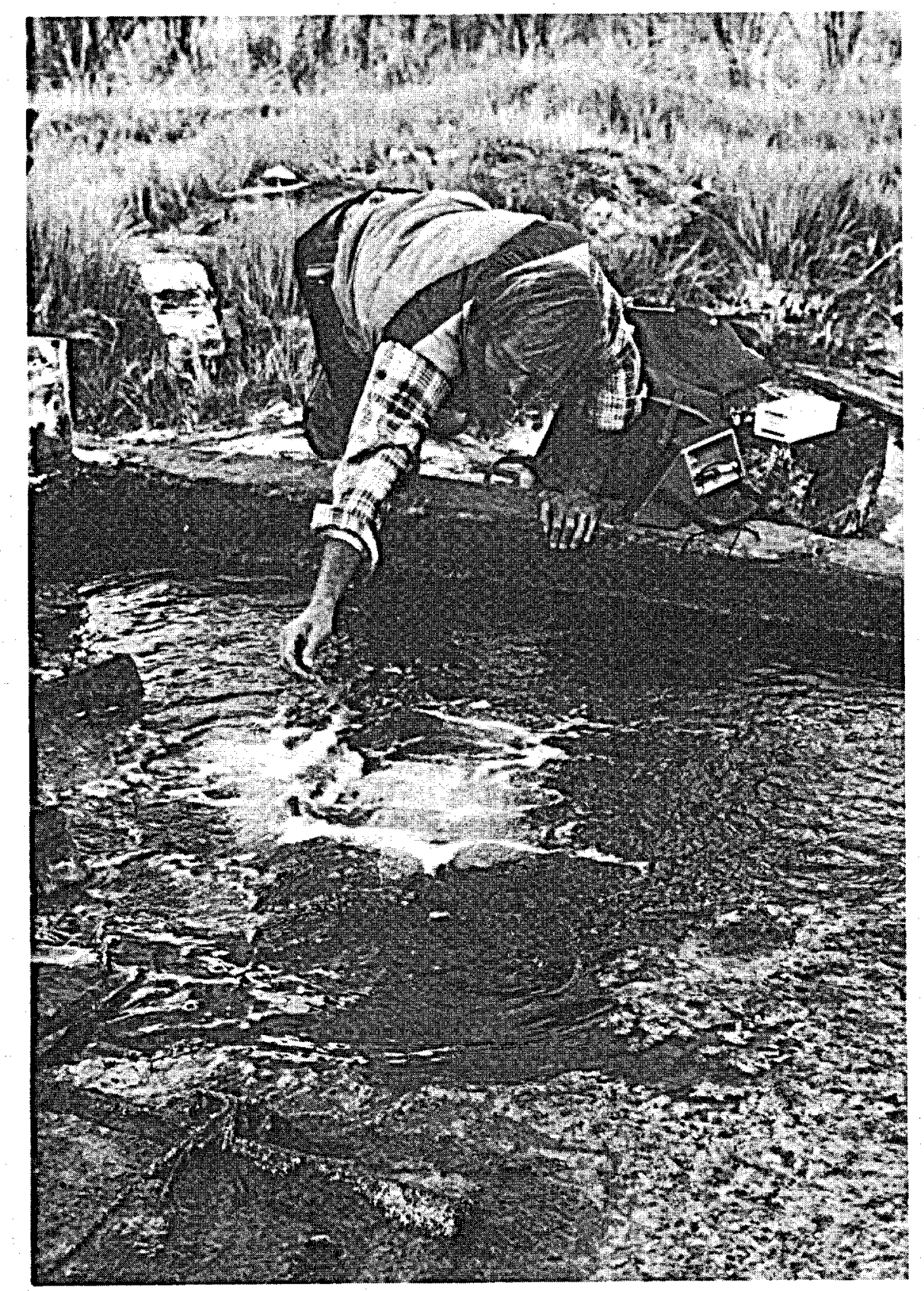

Fig. A-11.

Zia Hot Well (Kaseman \#2 0il Test Wel1) in April 1980; $\mathrm{pH} \simeq 6.5$, temperature $\simeq 54^{\circ} \mathrm{C}$. Well was drilled in 1926 as oil test but struck hot water instead. A hotel which was built adjacent to the well burned down in the 1950's. Now unused, this well could supply substantial geothermal space heat. 
TABLES OF FIELD, CHEMICAL, AND ISOTOPIC DATA

TABLE B-I

LOCATION, FIELD DATA, AND REFERENCES FOR WATERS IN THE JEMEZ MOUNTAINS REGION, NEW MEXICO

\begin{tabular}{|c|c|c|c|c|c|c|c|c|c|c|}
\hline $\begin{array}{l}\text { Map } \\
\text { No.a }\end{array}$ & Name & $\begin{array}{l}\text { Field } \\
\text { No. }\end{array}$ & Date & Location & $\begin{array}{l}\text { Water } \\
\text { Typeb }\end{array}$ & $\operatorname{Temp}_{\left({ }^{\circ} \mathrm{c}\right)}$ & $\underset{\mathrm{pH}}{\mathrm{Ffeld}}$ & $\begin{array}{l}\text { Flow Rate } \\
\text { l/min }\end{array}$ & Comments & Reference ${ }^{c}$ \\
\hline \multicolumn{11}{|c|}{ Los Alamos Area, Pajarito Plateau } \\
\hline 1 & $\begin{array}{l}\text { Gallery } \\
\text { Spring }\end{array}$ & LA-1 & $8 / 78$ & $\begin{array}{l}\text { SW-1/4, Sec } 25, \\
\text { T19N, R5E }\end{array}$ & m & 11 & 5.6 & 160 & $\begin{array}{l}\text { Spring Issues from concrete gallery } \\
\text { buflt in fractured Bandelier Tuff. }\end{array}$ & $\begin{array}{l}\text { G-S; G et al.; P79; } \\
T 78 \text { (R5T) }\end{array}$ \\
\hline 2 & T-3 Well & LA-2 & $8 / 78$ & $\begin{array}{l}\text { SH-1/4, Sec } 13, \\
\text { T19N, R6E }\end{array}$ & $m$ & 13 & 6.5 & $0-12$ & $\begin{array}{l}\text { observation Well }-50 \mathrm{~m} \text { deep, fron } \\
\text { casing; in alluvium and Puye(?)Fm. }\end{array}$ & G-S \\
\hline 3 & T-2 Well & LA-3 & $8 / 78$ & $\begin{array}{l}\text { NE-1/4, Sec } 14, \\
\text { T19N, R6E }\end{array}$ & $m$ & 11 & 5.7 & $0-8$ & $\begin{array}{l}\text { observation Well }-50 \mathrm{~m} \text { deep, fron } \\
\text { casing; in alluvium and Puye(?)Fin. }\end{array}$ & G-S \\
\hline 4 & $\begin{array}{l}\text { Sacred } \\
\text { Spring }\end{array}$ & LA-4 & $8 / 78$ & $\begin{array}{l}\text { Center, Sec } 12 \text {, } \\
\text { T19N, R7E }\end{array}$ & $m$ & 14 & 5.7 & Seep & Seep discharges from Santa Fe Fm. & G-S; G et al.; T78 (T6) \\
\hline 5 & $\begin{array}{l}\text { Basalt } \\
\text { Spring }\end{array}$ & LA-5 & $8 / 78$ & $\begin{array}{l}\text { NH-1/4, SeC 22, } \\
\text { T19N, R7E }\end{array}$ & m & 15 & 5.8 & 4 & $\begin{array}{l}\text { Spring flows from near contact of } \\
\text { Cerros del Rio basalt and Puye Fm. }\end{array}$ & G-S; G et al. \\
\hline 6 & L-6 Well & LA-6 & $9 / 78$ & $\begin{array}{l}\text { W-1/2, Sec 14, } \\
\text { T19N, R7E }\end{array}$ & m & 27 & 6.8 & $0-2160$ & $\begin{array}{l}\text { Water-supply well drilled } 625 \mathrm{~m} \text { into } \\
\text { Santa Fe Fm; steel casing. }\end{array}$ & G-S; Gr; P77; P79 \\
\hline 7 & L-1B Well & LA-7 & $9 / 78$ & $\begin{array}{l}\text { NW-1/4, Sec } 13, \\
\text { T19N, R7E }\end{array}$ & m & 30 & 7.2 & $0-2180$ & $\begin{array}{l}\text { Water-supply well drilled } 694 m \text { into } \\
\text { Santa Fe Fm; steel casing. }\end{array}$ & G-S; P77; P79; P-C \\
\hline 8 & L-5 Well & LA-8 & $9 / 78$ & $\begin{array}{l}\text { SE-1/4, Sec } 15, \\
\text { T19N, R7E }\end{array}$ & m & 26.5 & 6.5 & $0-1880$ & $\begin{array}{l}\text { Water-supply well drilled } 623 \text { m into } \\
\text { Santa Fe Fm; steel casing. }\end{array}$ & G-S; Gr; P77; P79; P-C \\
\hline 9 & L-4 Well & LA-9 & $9 / 78$ & $\begin{array}{l}\text { NH-1/4, Sec 22, } \\
\text { T19N, R7E }\end{array}$ & m & 28 & 6.5 & $0-1560$ & $\begin{array}{l}\text { Water-supply well drilled } 622 \text { in into } \\
\text { Santa Fe Fm; steel casing. }\end{array}$ & G-S; Gr; P77; P79 \\
\hline 10 & PM-2 Nell & LA-10 & $9 / 78$ & $\begin{array}{l}\text { NH-1/4, Sec } 36, \\
\text { T19N, R6E }\end{array}$ & m & 23.5 & 6.5 & $0-5520$ & $\begin{array}{l}\text { Nell drilled } 800 \mathrm{~m} \text { into Santa Fe Fm; } \\
\text { steel casing. }\end{array}$ & G-S; P79 \\
\hline 11 & PM-1 Well & LA-11 & $9 / 78$ & $\begin{array}{l}\text { SH-1/4, Sec 20, } \\
\text { T19N, R7E }\end{array}$ & m & 28 & 6.5 & $0-2320$ & $\begin{array}{l}\text { Well drilled } 770 \mathrm{~m} \text { into Santa Fe Fm; } \\
\text { steel casing. }\end{array}$ & G-S; P79 \\
\hline 12 & G-6 Well & LA-12 & $9 / 78$ & $\begin{array}{l}\text { NE-1/4, Sec } 6, \\
\text { T19N, R7E }\end{array}$ & $m$ & 30.5 & 6.5 & $0-1100$ & $\begin{array}{l}\text { Water-supply well drilled } 617 \mathrm{~m} \text { into } \\
\text { Santa Fe Fin; steel casing. }\end{array}$ & G-S; P79 \\
\hline 13 & G-5 Well & LA-13 & $9 / 78$ & $\begin{array}{l}\text { NW-1/4, Sec } 5 \text {, } \\
\text { T19N, R7E }\end{array}$ & $m$ & 26.5 & 6.5 & $0-2100$ & $\begin{array}{l}\text { Water-supply well drilled } 614 \mathrm{~m} \text { into } \\
\text { Santa Fe Fm; steel casing. }\end{array}$ & G-S; Gr; P79; P-C \\
\hline 14 & G-4 Well & LA-14 & $9 / 78$ & $\begin{array}{l}\text { Center, Sec 5, } \\
\text { T19N, R7E }\end{array}$ & $m$ & 26 & 6.5 & $0-1260$ & $\begin{array}{l}\text { Water-supply well drilled } 616 \text { in into } \\
\text { Santa Fe Fm; steel casing. }\end{array}$ & G-S; Gr; P79 \\
\hline 15 & G-3 Kell & LA-15 & $9 / 78$ & $\begin{array}{l}\text { NH-1/4, Sec } 4, \\
\text { T19N, R7E }\end{array}$ & $m$ & 29 & 6.5 & $0-1620$ & $\begin{array}{l}\text { Water-supply well drilled } 614 \mathrm{~m} \\
\text { into Santa } \mathrm{Fe} \mathrm{Fm} \text {; steel casing. }\end{array}$ & G-S; Gr; P79 \\
\hline
\end{tabular}


TABLE B-I (cont)

\begin{tabular}{|c|c|c|c|c|c|c|c|c|c|c|}
\hline $\begin{array}{l}\text { Map } \\
\text { Ho.a }\end{array}$ & Name & $\begin{array}{l}\text { Fteld } \\
\text { No. }\end{array}$ & Date & Location & $\begin{array}{l}\text { Water } \\
\text { Typeb }\end{array}$ & $\begin{array}{l}\text { Temp } \\
\left({ }^{\circ} \mathrm{C}\right)\end{array}$ & $\underset{\mathrm{pH}}{\text { Field } \mathrm{F}}$ & $\begin{array}{l}\text { low Rate, } \\
\ell / \mathrm{min}\end{array}$ & Comments & Reference $c$ \\
\hline 16 & G-2 Well & LA-16 & $9 / 78$ & $\begin{array}{l}\text { Center Sec } 4, \\
\text { TISN, R7E }\end{array}$ & m & 30 & 6.5 & $0-1820$ & $\begin{array}{l}\text { Water-supply well drilled } 617 \mathrm{~m} \\
\text { into Santa Fe Fm; steel casing. }\end{array}$ & G-S; Gr; P79 \\
\hline 17 & G-1A Hell & LA-17 & $9 / 78$ & $\begin{array}{l}\text { SE-1/4 Sec 4, } \\
\text { T19N, R7E }\end{array}$ & m & 28 & 6.5 & $0-2060$ & $\begin{array}{l}\text { Water-supply well drilled } 637 \mathrm{~m} \\
\text { into Santa Fe Fm; steel casing. }\end{array}$ & G-S; P79 \\
\hline 18 & G-1 Well & $L A-18$ & $9 / 78$ & $\begin{array}{l}\mathrm{SE}-1 / 4, \operatorname{Sec} 4, \\
\text { T19N, R7E }\end{array}$ & m & 26 & 6.5 & $0-1340$ & $\begin{array}{l}\text { Water-supply well drilled } 646 \mathrm{~m} \\
\text { into Santa Fe Fm; steel casing. }\end{array}$ & G-S; Gr; P79; P-C \\
\hline 19 & $\begin{array}{l}\text { Spring, White } \\
\text { Rock Canyon }\end{array}$ & LA-19 & $9 / 78$ & $\begin{array}{l}\text { NE-1/4, Sec } 36, \\
\text { T18N, R6E }\end{array}$ & m & 19 & 6.5 & 6 & $\begin{array}{l}\text { Spring issues from Santa Fe Fm; } \\
\text { covered by volcanic colluvium. }\end{array}$ & G-S; G et al.; T78 (K10) \\
\hline 20 & PM-3 Hell & $\angle A-20$ & $9 / 78$ & $\begin{array}{l}\mathrm{H}-1 / 2, \operatorname{Sec} 19, \\
\text { T19H, R7E }\end{array}$ & m & 27.5 & 6.5 & $0-5600$ & $\begin{array}{l}\text { Well drilled } 786 \text { m into Santa Fe } \\
\text { Fm; steel casing. }\end{array}$ & G-S; P79 \\
\hline \multicolumn{11}{|c|}{ Valles Caldera, Sulphur Springs Area } \\
\hline 21 & $\begin{array}{l}\text { Mudpot, Men's } \\
\text { Bathhouse }\end{array}$ & $V A-13$ & $1 / 79$ & $\begin{array}{l}\text { Lat. } 35^{\circ} 54^{\prime} \text {, } \\
\text { Long. } 106^{\circ} 37^{\prime} \\
\text { (Fig. } 3 \text { ) }\end{array}$ & a & 78 & 2.52 & 0 & $\begin{array}{l}\text { Mudpot in concrete crib in col- } \\
\text { lapsed bathhouse; from landslide } \\
\text { in rhyolite; } \mathrm{CO}_{2} \uparrow, \mathrm{H}_{2} \mathrm{St} \text {. }\end{array}$ & $G-G ; S ; R ; T 78$ (P2) \\
\hline 21 & $\begin{array}{l}\text { Mudpot, Men's } \\
\text { Bathhouse }\end{array}$ & $5-7-80$ & $9 / 80$ & $\begin{array}{l}\text { Lat. } 35^{\circ} 54^{\circ} \\
\text { Long. } 106^{\circ} 37^{\circ} \\
\text { (Fig. } 3)^{\circ}\end{array}$ & a & 82 & 2.0 & 0 & $\begin{array}{l}\text { Mudpot in concrete crib in col- } \\
\text { lapsed bathhouse; from landslide } \\
\text { in rhyolite; } \mathrm{CO}_{2}+, \mathrm{H}_{2} \mathrm{St} \text {. }\end{array}$ & \\
\hline 21 & $\begin{array}{l}\text { Unnamed Hot } \\
\text { Spring }\end{array}$ & VA-14 & $1 / 79$ & $\begin{array}{l}\text { Lat. } 35^{\circ} 54^{\prime} \\
\text { Long. } 106^{\circ} 37^{i} \\
\text { (Fig. } 3 \text { ) }\end{array}$ & a & 63 & 2.38 & 2 & $\begin{array}{l}\text { Spring issues from alluvium; } \\
\mathrm{CO}_{2}^{\dagger}, \mathrm{H}_{2} \mathrm{~S}^{\dagger} \text {. }\end{array}$ & G-G \\
\hline 21 & $\begin{array}{l}\text { Unnamed Hot } \\
\text { Spring. }\end{array}$ & $S-9-80$ & $9 / 80$ & $\begin{array}{l}\text { Lat. } 35^{\circ} 34^{\prime} \\
\text { Long. } 106^{\circ} 37^{\prime} \\
\text { (Fig. 3) }\end{array}$ & a & -- & 2.03 & 1 & $\begin{array}{l}\text { Spring issues from alluvium; } \\
\mathrm{CO}_{2}^{\uparrow}, \mathrm{H}_{2} \mathrm{~S}^{\dagger} \text {. }\end{array}$ & \\
\hline 21 & $\begin{array}{l}\text { Lemonade } \\
\text { Spring }\end{array}$ & $S-10-80$ & $9 / 80$ & $\begin{array}{l}\text { Lat. } 35^{\circ} 54^{\prime} \\
\text { Long.106 } \\
\text { (Ftg. 3) }\end{array}$ & a & 58 & 2.3 & $1 / 2$ & $\begin{array}{l}\text { Spring issues from alluvium; } \\
\mathrm{CO}_{2}^{\uparrow}, \mathrm{H}_{2} \mathrm{~S}^{\uparrow} \text {. }\end{array}$ & S, T78 (P1) \\
\hline 21 & $\begin{array}{l}\text { Unnamed } \\
\text { Spring }\end{array}$ & $S-3-80$ & $9 / 80$ & $\begin{array}{l}\text { Lat. } 35^{\circ} 54^{\prime} \\
\text { Long. } 106^{\circ} 37^{i} \\
\text { (Fig. 3) }\end{array}$ & $\mathrm{cm}$ & 11 & 3.6 & $1 / 4$ & $\begin{array}{l}\text { Spring discharges into Sulphur } \\
\text { Creek; } \mathrm{CO}_{2} \uparrow, \mathrm{H}_{2} \mathrm{St} \text {. }\end{array}$ & \\
\hline 21 & $\begin{array}{l}\text { Footbath } \\
\text { Spring }\end{array}$ & $5-4-80$ & $9 / 80$ & $\begin{array}{l}\text { Lat. } 35^{\circ} 54^{\prime} \\
\text { Long. } 106^{\circ} 37^{i} \\
\text { (Fig. } 3 \text { ) }\end{array}$ & a & 33 & 1.1 & 0 & $\begin{array}{l}\text { Large bubbling pool with colloidal } \\
\text { sulfer; } \mathrm{CO}_{2}^{+}, \mathrm{H}_{2} \mathrm{St} \text {. }\end{array}$ & $S$ \\
\hline 21 & $\begin{array}{l}\text { Electric } \\
\text { Spring }\end{array}$ & $S-5-80$ & $9 / 80$ & $\begin{array}{l}\text { Lat. } 35^{\circ} 54^{\prime} \\
\text { Long. } 106^{\circ} 37^{i} \\
\text { (Fig. } 3 \text { ) }\end{array}$ & $\mathbf{a}$ & 36 & 1.5 & $1 / 2$ & $\begin{array}{l}\text { Spring flows from alluvium; } \\
\mathrm{CO}_{2}{ }^{\dagger}, \mathrm{H}_{2} \mathrm{~S} \uparrow\end{array}$ & $s$ \\
\hline
\end{tabular}




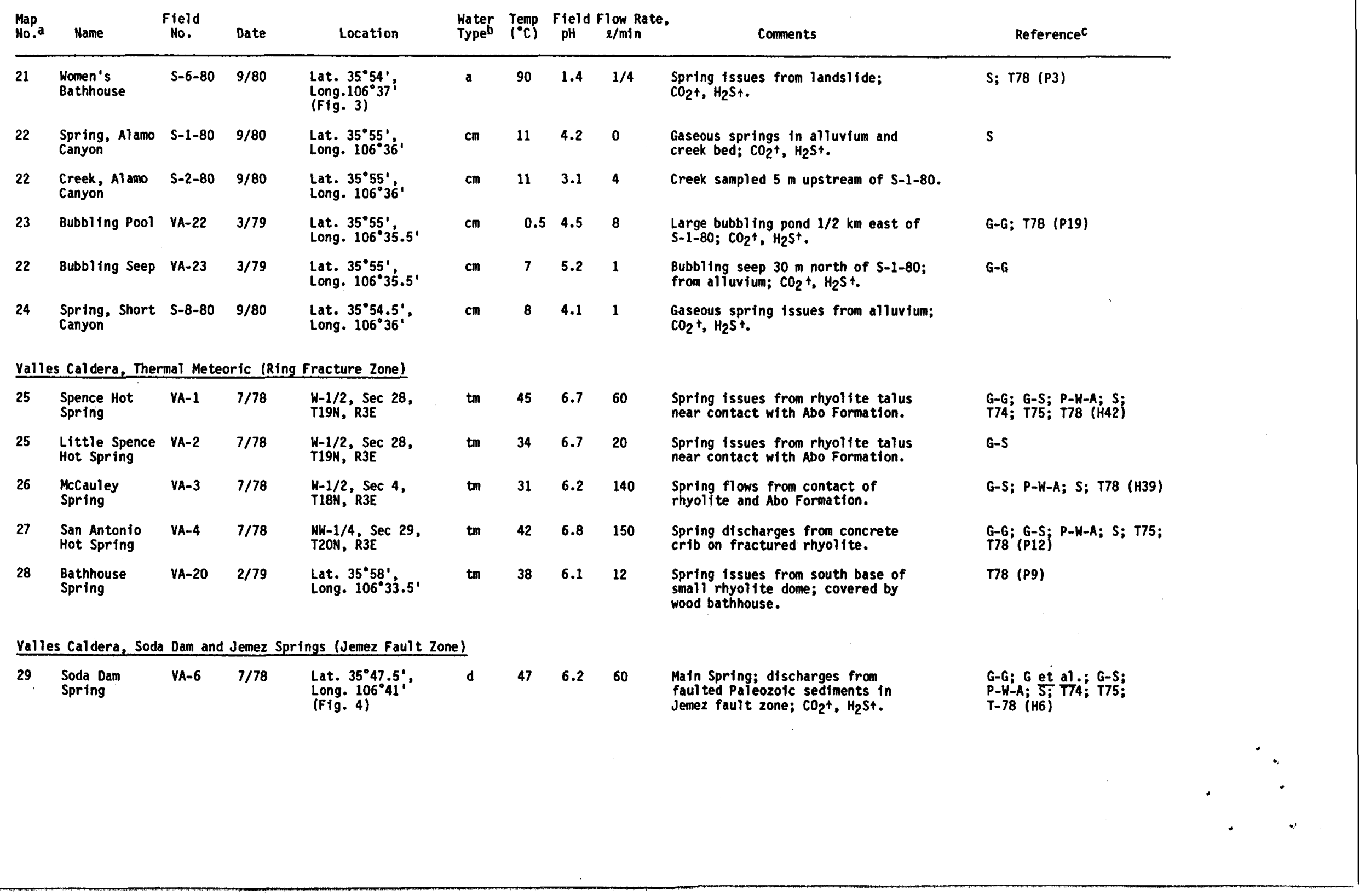


TABLE B-I (cont)

\begin{tabular}{|c|c|c|c|c|c|c|c|c|c|c|}
\hline $\begin{array}{l}\text { Map } \\
\text { No.a }\end{array}$ & Name & $\begin{array}{l}\text { Field } \\
\text { No. }\end{array}$ & Date & Location & $\begin{array}{l}\text { Mater } \\
\text { Type }\end{array}$ & $\begin{array}{l}\text { Temp } \\
\left({ }^{\circ} \mathrm{C}\right)\end{array}$ & $\underset{\mathrm{pH}}{\text { Field }}$ & $\begin{array}{l}\text { Flow Rate, } \\
\text { l/min }\end{array}$ & Comments & Referencec \\
\hline 29 & $\begin{array}{l}\text { Soda Dam } \\
\text { Spring }\end{array}$ & VA-9 & $1 / 79$ & $\begin{array}{l}\text { Lat. } 35^{\circ} 47.5{ }^{\prime}, \\
\text { Long. } 106^{\circ} 41^{\prime} \\
\text { SFig. } 4)^{\circ}\end{array}$ & d & 48 & 6.40 & 60 & $\begin{array}{l}\text { Main Spring; discharges from } \\
\text { faulted Paleozoic sediments in } \\
\text { Jemez fault zone; } \mathrm{CO}_{2}+\mathrm{H}_{2} \mathrm{St} \text {. }\end{array}$ & \\
\hline 29 & $\begin{array}{l}\text { Soda Dam } \\
\text { Spring }\end{array}$ & YA-64 & $12 / 80$ & $\begin{array}{l}\text { Lat. } 35^{\circ} 47.5^{\prime}, \\
\text { Long. } 106^{\circ} 41^{\prime} \\
\text { (Fig. } 4)\end{array}$ & d & 47 & 6.28 & 60 & $\begin{array}{l}\text { Main Spring; discharges from } \\
\text { faulted Paleozoic sediments in } \\
\text { Jemez fault zone; } \mathrm{CO}_{2}^{+}, \mathrm{H}_{2} \mathrm{~S}^{\dagger} \text {. }\end{array}$ & \\
\hline 29 & $\begin{array}{l}\text { Soda Dam } \\
\text { Spring }\end{array}$ & VA-26 & $5 / 79$ & $\begin{array}{l}\text { Lat. } 35^{\circ} 47.5^{\prime}, \\
\text { Long. } 106^{\circ} 41^{\prime} \\
\text { (Fig. } 4)^{\prime}\end{array}$ & d & 47 & 6.52 & 60 & $\begin{array}{l}\text { Main Spring; discharges from } \\
\text { faul ted Paleozoic sediments in } \\
\text { Jemez fault zone; } \mathrm{CO}_{2}{ }^{4}, \mathrm{H}_{2} \mathrm{St}^{4} \text {. }\end{array}$ & \\
\hline 29 & $\begin{array}{l}\text { Soda Dam } \\
\text { Spring }\end{array}$ & VA-51 & $4 / 80$ & $\begin{array}{l}\text { Lat. } 35^{\circ} 47.5^{\prime}, \\
\text { Long. } 106^{\circ} 41^{\prime} \\
\text { (Fig. } 4)^{\prime}\end{array}$ & d & 47 & 6.35 & 60 & $\begin{array}{l}\text { Main Spring; discharges from } \\
\text { faul ted Paleozoic sediments in } \\
\text { Jemez fault zone; } \mathrm{CO}_{2}{ }^{+}, \mathrm{H}_{2} \mathrm{~S}^{\dagger} \text {. }\end{array}$ & \\
\hline 29 & $\begin{array}{l}\text { Grotto } \\
\text { Spring }\end{array}$ & $V A-5$ & $7 / 78$ & $\begin{array}{l}\text { Lat. } 35^{\circ} 47.5^{\prime}, \\
\text { Long. } 106^{\circ} 41^{\prime} \\
\text { (Fig. 4) }\end{array}$ & $d$ & 38 & 6.8 & 12 & $\begin{array}{l}\text { Spring flows from cave on east } \\
\text { side Soda Dam. }\end{array}$ & G-S \\
\hline 29 & $\begin{array}{l}\text { Outfall of } \\
\text { Soda Dam } \\
\text { Spring }\end{array}$ & $v A-65$ & $12 / 80$ & $\begin{array}{l}\text { Lat. } 35^{\circ} 47.5^{\prime}, \\
\text { Long. } 106^{\circ} 41^{\prime} \\
\text { (Fig. } 4)\end{array}$ & d & 17 & 8.13 & 60 & $\begin{array}{l}\text { Sampled at point where water of } \\
\text { Soda Dam Spring enters Jemez } \\
\text { River. }\end{array}$ & \\
\hline 29 & $\begin{array}{l}\text { Unnamed } \\
\text { Spring }\end{array}$ & VA-27 & $5 / 79$ & $\begin{array}{l}\text { Lat. } 35^{\circ} 47.5^{\prime} \text {, } \\
\text { Long. } 106^{\circ} 41^{\prime} \\
\text { (Fig. } 4)^{\prime}\end{array}$ & d & 29 & 6.28 & 2 & $\begin{array}{l}\text { Spring issues from alluvium } \\
\text { east side Jemez River. }\end{array}$ & \\
\hline 30 & $\begin{array}{l}\text { Main Jemez } \\
\text { Spring }\end{array}$ & VA-10 & $1 / 79$ & $\begin{array}{l}\text { Lat. } 35^{\circ} 46.5^{\prime}, \\
\text { Long. } 106^{\circ} 41^{\prime} \\
\text { (Fig. } 5 \text { ) }\end{array}$ & $d$ & 55 & 7.01 & 20 & $\begin{array}{l}\text { Spring discharges from concrete } \\
\text { tank under gazebo; } \mathrm{CO}_{2}^{+} \text {. }\end{array}$ & $\begin{array}{l}\text { G-G; } \underset{T 78}{G} \text { et } \text { al-; T75) } \\
\text { (H15) }\end{array}$ \\
\hline 30 & $\begin{array}{l}\text { Main Jemez } \\
\text { Spring }\end{array}$ & VA-18 & $1 / 79$ & $\begin{array}{l}\text { Lat. } 35^{\circ} 46.5^{\prime}, \\
\text { Long. } 106^{\circ} 41^{\circ} \\
\text { (Fig. } 5 \text { ) }\end{array}$ & d & 36 & 7.51 & 0 & $\begin{array}{l}\text { Spring discharges from concrete } \\
\text { tank under gazebo; } \mathrm{CO}_{2}{ }^{\dagger} \text {. }\end{array}$ & $G$ et al. \\
\hline 30 & $\begin{array}{l}\text { Travertine } \\
\text { Mound Spring }\end{array}$ & VA-7 & $1 / 79$ & $\begin{array}{l}\text { Lat. } 35^{\circ} 46.5^{\prime}, \\
\text { Long. } 106^{\circ} 41^{\prime} \\
\text { (Fig. } 5 \text { ) }\end{array}$ & d & 70 & 6.28 & 4 & $\begin{array}{l}\text { Spring issues from travertine } \\
\text { mound west of bathhouse; } \cdot \mathrm{CO}_{2}+\text {. }\end{array}$ & $G$ et al.; T74; T78(H14) \\
\hline 30 & $\begin{array}{l}\text { Travertine } \\
\text { Mound Spring }\end{array}$ & VA-66 & $12 / 80$ & $\begin{array}{l}\text { Lat. } 35^{\circ} 46.5^{\prime}, \\
\text { Long. } 106^{\circ} 41^{\circ} \\
\text { (fig. } 5 \text { ) }\end{array}$ & d & 72 & 6.23 & 4 & $\begin{array}{l}\text { Spring issues from travertine } \\
\text { mound west of bathhouse; } \mathrm{CO}_{2}^{\dagger} \text {. }\end{array}$ & $G$ et al. \\
\hline 30 & $\begin{array}{l}\text { Travertine } \\
\text { Mound Spring }\end{array}$ & VA-17 & $1 / 79$ & $\begin{array}{l}\text { Lat. } 35^{\circ} 46.5^{\prime}, \\
\text { Long. } 106^{\circ} 41^{\prime} \\
\text { (Fig. 5) }\end{array}$ & $d$ & 72 & 6.66 & 4 & $\begin{array}{l}\text { Spring issues from travertine } \\
\text { mound west of bathhouse; } \mathrm{CO}_{2}{ }^{4} \text {. }\end{array}$ & $G$ et al. \\
\hline 30 & $\begin{array}{l}\text { Buddhist } \\
\text { Spring }\end{array}$ & VA-8 & $1 / 79$ & $\begin{array}{l}\text { Lat. } 35^{\circ} 46.5^{\prime}, \\
\text { Long. } 106^{\circ} 41^{\prime} \\
\text { (Fig. } 5 \text { ) }\end{array}$ & d & 49 & 6.38 & 4 & $\begin{array}{l}\text { Spring flows from man-made pool } \\
\text { by Jemez River; } \mathrm{CO}_{2}^{\dagger} \text {. }\end{array}$ & $G$ et al. \\
\hline
\end{tabular}




\begin{tabular}{|c|c|c|c|c|c|c|c|c|c|c|}
\hline $\begin{array}{l}\text { Map } \\
\text { No.a }\end{array}$ & Name & $\begin{array}{l}\text { Field } \\
\text { No. }\end{array}$ & Date & Location & $\begin{array}{l}\text { Water } \\
\text { Typeb }\end{array}$ & $\begin{array}{l}\text { Temp } \\
\left({ }^{\circ} \mathrm{c}\right)\end{array}$ & $\begin{array}{l}\text { Field } \\
\text { pH }\end{array}$ & $\begin{array}{l}\text { Flow Rate, } \\
\mathrm{l} / \mathrm{min}\end{array}$ & Comments & Referencec \\
\hline 30 & $\begin{array}{l}\text { Buddhist } \\
\text { Spring }\end{array}$ & VA-16 & $1 / 79$ & $\begin{array}{l}\text { Lat. } 35^{\circ} 46.5^{\prime} \text {, } \\
\text { Long. } 106^{\circ} 41^{\prime}\end{array}$ & d & 50 & 6.59 & 4 & $\begin{array}{l}\text { Spring flows from man-made pool by } \\
\text { Jemez River; } \mathrm{CO}_{2}{ }^{+} \text {. }\end{array}$ & \\
\hline 30 & $\begin{array}{l}\text { Unnamed } \\
\text { Spring }\end{array}$ & VA-12 & $1 / 79$ & $\begin{array}{l}\text { Lat. } 35^{\circ} 46.5^{\prime} \text {, } \\
\text { Long. } 106^{\circ} 41^{\prime}\end{array}$ & d & 49 & 6.35 & 4 & $\begin{array}{l}\text { Spring discharged from marsh } 15 \text { m NW } \\
\text { of main Jemez Spring; } \mathrm{CO}_{24} \text { (now de- } \\
\text { stroyed). }\end{array}$ & \\
\hline 30 & $\begin{array}{l}80 \mathrm{ft} \\
\text { Aquifer }\end{array}$ & VA-19 & $1 / 79$ & $\begin{array}{l}\text { Lat. } 35^{\circ} 46.5^{\prime}, \\
\text { Long. } 106^{\circ} 41^{\prime}\end{array}$ & d & 68 & 6.64 & 120 & Well drilled $N$ of main Jemez Spring & $G$ et al. \\
\hline 30 & $\begin{array}{l}80 \mathrm{ft} \\
\text { Aquifer }\end{array}$ & VA-25 & $5 / 79$ & $\begin{array}{l}\text { Lat. } 35^{\circ} 46.5^{\prime} \text {, } \\
\text { Long. } 106^{\circ} 41^{\prime}\end{array}$ & d & 73.3 & 6.55 & 8 & Well drilled $N$ of main Jemez Spring & \\
\hline 30 & $\begin{array}{l}500 \mathrm{ft} \\
\text { Aquifer }\end{array}$ & $V A-15$ & $1 / 79$ & $\begin{array}{l}\text { Lat. } 35^{\circ} 46.5^{\prime}, \\
\text { Long. } 106^{\circ} 41^{\prime}\end{array}$ & $d$ & 60.5 & 6.69 & 80 & Well drilled $N$ of main Jemez Spring & $G$ et al. \\
\hline 30 & $\begin{array}{l}500 \mathrm{ft} \\
\text { Aquifer }\end{array}$ & VA-21 & $2 / 79$ & $\begin{array}{l}\text { Lat. } 35^{\circ} 46.5^{\prime}, \\
\text { Long. } 106^{\circ} 41^{\prime}\end{array}$ & d & 61 & 6.55 & 20 & Well drilled N of main Jemez Spring & \\
\hline \multicolumn{11}{|c|}{ Valles Caldera Region, Miscellaneous } \\
\hline 28 & $\begin{array}{l}\text { San Antonio } \\
\text { Creek }\end{array}$ & VA-24 & $2 / 79$ & $\begin{array}{l}\text { Lat. } 35^{\circ} 58^{\prime}, \\
\text { Long. } 106^{\circ} 33^{\prime} 5^{\prime}\end{array}$ & $m$ & 2 & $\cdots$ & $>250$ & $\begin{array}{l}\text { Creek sampled } 5 \mathrm{~m} \text { upstreaw of VA-20, } \\
\text { Bathhouse Spring. }\end{array}$ & \\
\hline 31 & $\begin{array}{l}\text { Panorama } \\
\text { Spring }\end{array}$ & VA-28 & $5 / 79$ & $\begin{array}{l}\text { Lat. } 35^{\circ} 48^{\prime} \\
\text { Long. } 106^{\circ} 41^{\prime}\end{array}$ & $m$ & 13 & 7.67 & 4 & $\begin{array}{l}\text { Spring issues from travertine and } \\
\text { Madera. Iimestone, } 100 \mathrm{~m} \text { above canyon- } \\
\text { floor. }\end{array}$ & . \\
\hline 32 & $\begin{array}{l}\text { Pajarito } \\
\text { Spring }\end{array}$ & VA-29 & $7 / 79$ & $\begin{array}{l}\text { SE-1/4, Sec } 9, \\
\text { T18N, R7E }\end{array}$ & $m$ & 20 & 5.9 & $>300$ & $\begin{array}{l}\text { Spring issues from landslide in } \\
\text { basait; spring } 4 A \text { of Purtymun. }\end{array}$ & P-P-0; T78 (K4) \\
\hline 33 & $\begin{array}{l}\text { Spring, White } \\
\text { Rock Canyon }\end{array}$ & VA-30 & $7 / 79$ & $\begin{array}{l}\text { W-1/2, Sec } 10 \\
\text { T18N, R7E }\end{array}$ & $m$ & 18 & 6.0 & 1 & $\begin{array}{l}\text { Spring issues from alluvium west } \\
\text { of Rio Grande; spring } 4 \text { of Purtymun. }\end{array}$ & P-P-0; T78 (K5) \\
\hline 34 & $\begin{array}{l}\text { Cold Mineral } \\
\text { Seep }\end{array}$ & VA-31 & $8 / 79$ & $\begin{array}{l}\text { SH-1/4, Sec 32, } \\
\text { T19N, R3E }\end{array}$ & $\mathbb{m}$ & 19 & 8.37 & 1 & $\begin{array}{l}\text { Seep about } 1 / 4 \mathrm{~km} \text { WNH of Battleship } \\
\text { Rock and Just } \mathrm{W} \mathrm{highway} 4 \text {; from } \\
\text { Madera Iimes tone. }\end{array}$ & \\
\hline 35 & Sino Spring & VA-32 & $8 / 79$ & $\begin{array}{l}\text { Lat. } 35^{\circ} 49^{\prime} \\
\text { Long. } 106^{\circ} 41^{\prime}\end{array}$ & $m$ & 21 & 7.45 & 15 & $\begin{array}{l}\text { Spring flows from contact of andesite } \\
\text { and Abo formation. }\end{array}$ & P-H-A; T78 (H1) \\
\hline 35 & Sino Spring & YA-63 & $12 / 80$ & $\begin{array}{l}\text { Lat. } 35^{\circ} 49^{\prime} \\
\text { Long. } 106^{\circ} 41^{\prime}\end{array}$ & $\mathbf{m}$ & 18 & 7.10 & 80 & $\begin{array}{l}\text { Spring flows from contact of andesite } \\
\text { and Abo Formation. }\end{array}$ & \\
\hline $36 d$ & $\begin{array}{l}\text { Unnamed } \\
\text { Spring }\end{array}$ & VA-39 & $3 / 81$ & $\begin{array}{l}\text { SE-1/4, Sec } 10, \\
\text { T16N, R2E }\end{array}$ & c & 11 & 6.91 & seep & $\begin{array}{l}\text { Spring discharges from Chinie Fm. } \\
\text { near contact w/overlying Zia SS; } \\
\text { tastes salty. }\end{array}$ & $T 78$ (E1) \\
\hline
\end{tabular}


TABLE B-I (cont)

\begin{tabular}{|c|c|c|c|c|c|c|c|c|c|c|}
\hline $\begin{array}{l}\text { Map. } \\
\text { No.a }\end{array}$ & Name & $\begin{array}{l}\text { Field } \\
\text { No. }\end{array}$ & Date & Location & $\begin{array}{l}\text { Water } \\
\text { Type }\end{array}$ & Temp & $\underset{\mathrm{pH}}{\text { Field } \mathrm{F}}$ & $\begin{array}{l}\text { Flow Rate, } \\
\ell / \min \end{array}$ & Comments & Reference $c$ \\
\hline $37^{d}$ & Canon Spring & VA- 40 & 4/81 & $\begin{array}{l}\text { Lat. } 35^{\circ} 40.5^{\circ} \\
\text { Long. } 106^{\circ} 45.5^{\prime}\end{array}$ & m & 18 & 7.58 & 1 & $\begin{array}{l}\text { Spring Issues from fault in Precam- } \\
\text { brian granite. }\end{array}$ & $T 78(06)$ \\
\hline $38^{\mathrm{d}}$ & $\begin{array}{l}\text { Indfian Valley } \\
\text { Well }\end{array}$ & $V A-41$ & $10 / 79$ & $\begin{array}{l}\text { SE-1/4, Sec } 11, \\
\text { T18N, R3E }\end{array}$ & m & 17.5 & 6.81 & 0 & $\begin{array}{l}\text { Well drilled } 60+\mathrm{m} \text { in alluvium and } \\
\text { pumice; tron casing. }\end{array}$ & \\
\hline $39 \mathrm{~d}$ & $\begin{array}{l}\text { Unnamed cold } \\
\text { Spring }\end{array}$ & $V A=42$ & $10 / 79$ & $\begin{array}{l}\text { Lat. } 35^{\circ} 51.5^{1} \\
\text { Long. } 106^{\circ} 27^{\prime}\end{array}$ & m & 15 & 7.20 & 30 & $\begin{array}{l}\text { Spring issues from rhyolite colluvium; } \\
\text { elev. }=8606 \mathrm{ft} .\end{array}$ & \\
\hline 40d & $\begin{array}{l}\text { Unnamed cold } \\
\text { Spring }\end{array}$ & VA-43 & $10 / 79$ & $\begin{array}{l}\text { SH-1/4, Sec 10; } \\
\text { T18N, R4E }\end{array}$ & m & 15 & 6.68 & 8 & $\begin{array}{l}\text { Spring flows from dacite colluvium; } \\
\text { elev. }=9100 \mathrm{ft} .\end{array}$ & \\
\hline a1d & $\begin{array}{l}\text { Unnamed cold } \\
\text { Spring }\end{array}$ & $V A-44$ & $10 / 79$ & $\begin{array}{l}\text { E-1/2, Sec 22, } \\
\text { T18N, R3E }\end{array}$ & m & 8 & 6.91 & 2 & $\begin{array}{l}\text { Spring discharges from rock crib on } \\
\text { andesite allurium by dirt road. }\end{array}$ & T78 (J2) \\
\hline $42^{d}$ & $\begin{array}{l}\text { Horseshoe } \\
\text { Spring }\end{array}$ & $V A-45$ & $10 / 79$ & $\begin{array}{l}\text { E-1/2, Sec 18, } \\
\text { T19N, R3E }\end{array}$ & $m$ & 12 & 6.89 & 12 & Spring issues from rhyolite alluvium. & $P-H-A ; T 78$ (N15) \\
\hline $43^{d}$ & $\begin{array}{l}\text { Unnamed cold } \\
\text { Spring }\end{array}$ & VA-46 & $10 / 79$ & $\begin{array}{l}\text { Lat. } 35^{\circ} 52.5^{\circ} \\
\text { Long, } 106^{\circ} 44 . .^{\prime}\end{array}$ & $m$ & 10 & 6.42 & 40 & $\begin{array}{l}\text { Spring flows from contact of } \\
\text { Bandeleer Tuff and Abo formation; } \\
\text { sampled from fron pipe. }\end{array}$ & 778 (N6) \\
\hline 44d & $\begin{array}{l}\text { Unnamed cold } \\
\text { Spring }\end{array}$ & VA-47 & $10 / 79$ & $\begin{array}{l}\text { NE-1/4, SeC 27, } \\
\text { T2ON, R2E }\end{array}$ & m & 10 & 6.48 & 60 & $\begin{array}{l}\text { Spring discharges from fractured } \\
\text { Bandelier Tuff. }\end{array}$ & $\mathrm{T} 78(\mathrm{M10})$ \\
\hline 45 & $\begin{array}{l}\text { Unnamed cold } \\
\text { Spring }\end{array}$ & $\mathrm{YA}-48$ & $6 / 80$ & $\begin{array}{l}\text { Lat. } 35^{\circ} 45^{\prime} \\
\text { Long. } 106^{\circ} 28^{\prime}\end{array}$ & $m$ & 9 & 5.6 & 12 & $\begin{array}{l}\text { Spring issues from gully } 1 / 2 \mathrm{~km} \text { east } \\
\text { of Albemarle ruins; from hypabyssal } \\
\text { volcanics. }\end{array}$ & \\
\hline 46 & Eddy's Hell & VA-49 & $6 / 80$ & $\begin{array}{l}\text { Center, Sec 16, } \\
\text { I19N, R3E }\end{array}$ & $m$ & 15 & 7.24 & $0-20$ & Well $40+m$ deep in alluvilum and pumice. & \\
\hline 34 & $\begin{array}{l}\text { Cold Mineral } \\
\text { Seep }\end{array}$ & $V A-50$ & $4 / 80$ & $\begin{array}{l}\text { SH-1/4, Sec 32, } \\
\text { T19N, R3E }\end{array}$ & $m$ & 11 & 7.92 & 1. & $\begin{array}{l}\text { Seep about } 1 / 4 \mathrm{~km} \text { WW Battleship Rock } \\
\text { and Just W Highway 4; from Madera } 1 \text { ime- } \\
\text { stone. }\end{array}$ & \\
\hline 29 & Jemez River & VA-52 & $4 / 80$ & $\begin{array}{l}\text { Lat. } 35^{\circ} 47.5^{\circ}, \\
\text { Long. } 105^{\circ} 41^{\circ}\end{array}$ & m & 5 & 5.30 & $\gg>500$ & $\begin{array}{l}\text { River sampled } 10 \mathrm{~m} \text { upstream of } \\
\text { Soda Dam travertine deposit; } \\
\text { high water. }\end{array}$ & $\mathrm{P}-\mathrm{H}-\mathrm{A} ; \mathrm{T78}$ \\
\hline 48 & $\begin{array}{l}\text { Unnamed cold } \\
\text { Spring }\end{array}$ & VA-54 & $6 / 80$ & $\begin{array}{l}N-1 / 2, \operatorname{Sec} 13, \\
\text { T18N, RAE. }\end{array}$ & $m$ & 11 & 5.4 & 20 & $\begin{array}{l}\text { Spring issues from alluvium in } \\
\text { canon del Norte. }\end{array}$ & \\
\hline 49 & $\begin{array}{l}\text { Unnamed cold } \\
\text { Spring }\end{array}$ & VA-55 & $6 / 80$ & $\begin{array}{l}\text { Lat. } 35^{\circ} 51^{\prime} \\
\text { Long. } 106^{\circ} 25.5^{\prime}\end{array}$ & m & 8.5 & 5.4 & $20^{\circ}$ & $\begin{array}{l}\text { Spring issues from contact of latite } \\
\text { and Bandelier Tuff. }\end{array}$ & \\
\hline 50 & $\begin{array}{l}\text { Unnamed cold } \\
\text { Spring }\end{array}$ & VA-56 & $6 / 80$ & $\begin{array}{l}\text { Lat. } 35^{\circ} 50^{\prime} \\
\text { Long. } 106^{\circ} 24.5^{\prime}\end{array}$ & m & 6.5 & 5.4 & 24 & $\begin{array}{l}\text { Spring discharges from contact of } \\
\text { latite and Bandel fer Tuff. }\end{array}$ & \\
\hline
\end{tabular}




\begin{tabular}{|c|c|c|c|c|c|c|c|c|c|c|}
\hline $\begin{array}{l}\text { Map } \\
\text { No.a }\end{array}$ & Name & $\begin{array}{l}\text { Field } \\
\text { No. }\end{array}$ & Date & Location & $\begin{array}{l}\text { Water } \\
\text { Typeb }\end{array}$ & $\begin{array}{l}\text { Temp } \\
\left({ }^{\circ} \mathrm{C}\right)\end{array}$ & $\begin{array}{l}\text { Field } \\
\text { pH }\end{array}$ & $\begin{array}{l}\text { Flow Rate, } \\
\ell / \text { min }\end{array}$ & Comments & Referencec \\
\hline 51 & Apache Spring & VA-57 & $7 / 80$ & $\begin{array}{l}\text { Lat. } 35^{\circ} 49^{\prime}, \\
\text { Long. } 106^{\circ} 23.5^{\prime}\end{array}$ & $m$ & 9 & 5.3 & 15 & $\begin{array}{l}\text { Spring discharges from contact of } \\
\text { latite and Bandelier Tuff. }\end{array}$ & \\
\hline 52 & $\begin{array}{l}\text { Unnamed Cold } \\
\text { Spring }\end{array}$ & VA-58 & $7 / 80$ & $\begin{array}{l}\text { Lat. } 35^{\circ} 48^{\prime} \\
\text { Long. } 106^{\circ} 20^{\prime}\end{array}$ & $\mathbf{m}$ & 15 & 5.4 & 12 & $\begin{array}{l}\text { Spring flows from fractured Bandelier } \\
\text { Tuff. }\end{array}$ & \\
\hline 53 & $\begin{array}{l}\text { Unnamed Cold } \\
\text { Spring }\end{array}$ & VA-59 & $7 / 80$ & $\begin{array}{l}\text { Lat. } 35^{\circ} 48^{\prime} \\
\text { Long. } 106^{\circ} 20^{\prime}\end{array}$ & m & 17 & 5.6 & 24 & $\begin{array}{l}\text { Spring issues from fractured Bandelier } \\
\text { Tuff. }\end{array}$ & \\
\hline 54 & Turkey Springs & $V A-60$ & $7 / 80$ & $\begin{array}{l}\text { Lat. } 35^{\circ} 44.5^{\prime} \\
\text { Long. } 106^{\circ} 21^{\prime} 5^{\prime}\end{array}$ & $m$ & 18 & 5.5 & 60 & Spring flows from volcanic alluvium. & \\
\hline 46 & Henson's Well & $V A-61$ & $12 / 80$ & $\begin{array}{l}\text { Center Sec } 16, \\
\text { T19N, R3E }\end{array}$ & m & 19 & 6.82 & $0-40$ & $\begin{array}{l}\text { Well drilled } 65 \text { m into caldera fill; } \\
\text { plastic pipe. }\end{array}$ & \\
\hline
\end{tabular}

\section{San Ysidro - Jemez Pueblo Area, Miscell aneous}

\begin{tabular}{|c|c|c|c|c|c|c|c|c|c|c|}
\hline 55 & $\begin{array}{l}\text { Warm Mineral } \\
\text { Spring }\end{array}$ & $V A-33$ & $8 / 79$ & $\begin{array}{l}\text { NW-1/4, Sec 10, } \\
\text { TISN, RIE }\end{array}$ & c & 27 & 6.57 & 1 & $\begin{array}{l}\text { Spring seeps from travertine mound } \\
\text { N of highway } 44 ; \mathrm{Chinle} \mathrm{Fm} ; \mathrm{CO}_{2}^{+} \text {. }\end{array}$ & $T 74() ; T 78$ ( ); M \\
\hline 56 & Zia Hot Well & VA-34 & $8 / 79$ & $\begin{array}{l}\text { Lat. } 35^{\circ} 39^{\prime} \\
\text { Long. } 106^{\circ} 53^{\prime}\end{array}$ & c & 56 & 6.29 & 40 & $\begin{array}{l}\text { Artesian well flows from concrete } \\
\text { crib E of highway 44; Chinle } \mathrm{Fm} ; \mathrm{CO}_{2}+\text {. }\end{array}$ & T74; T78 (C3) \\
\hline 56 & Zia Hot Well & VA-52 & $4 / 80$ & $\begin{array}{l}\text { Lat. } 35^{\circ} 39^{\prime} \\
\text { Long. } 106^{\circ} 53^{\prime}\end{array}$ & c & 54 & 6.53 & 40 & $\begin{array}{l}\text { Artesian well flows from concrete } \\
\text { crib E of highway 44; Chinle } \mathrm{Fm}_{3} \mathrm{CO}_{2} \uparrow \text {. }\end{array}$ & \\
\hline 56 & Zia Hot Well & VA-67 & $3 / 81$ & $\begin{array}{l}\text { Lat. } 35^{\circ} 39^{\prime}, \\
\text { Long. } 106^{\circ} 53^{\prime}\end{array}$ & c & 53 & 6.72 & 40 & $\begin{array}{l}\text { Artesian well flows from concrete } \\
\text { crib E of highway } 44 \text {; Chinle } \mathrm{Fm} \mathrm{CO} \mathrm{CO}_{2}+\end{array}$ & \\
\hline $57^{d}$ & Unnamed Well & VA-35 & $8 / 79$ & $\begin{array}{l}\text { Lat. } 35^{\circ} 42.5^{\prime} \\
\text { Long. } 106^{\circ} 59.5^{\prime}\end{array}$ & m & 21 & -- & $1 / 2$ & $\begin{array}{l}\text { Well drains into cattle trough above } \\
\text { San Luis Tank; Morrison Fm(?). }\end{array}$ & \\
\hline $58 d$ & Salt Spring & VA-36 & $3 / 81$ & $\begin{array}{l}\text { SH-1/4, Sec 20, } \\
\text { T16N, R2E }\end{array}$ & c & 15.5 & 7.90 & $1 / 2$ & $\begin{array}{l}\text { Spring seeps from Chinle Fm and } \\
\text { Tertiary gravels. }\end{array}$ & T78 (A10) \\
\hline $59 \mathrm{~d}$ & Log Spring & VA-37 & $8 / 79$ & $\begin{array}{l}\text { E-1/2, Sec 5, } \\
\text { T16N, R1E }\end{array}$ & m & 28.5 & -- & $<1$ & $\begin{array}{l}\text { Spring seeps from fault zone near } \\
\text { contact of } 1 \text { imes tone and granite. }\end{array}$ & T78 (D4) \\
\hline $60^{d}$ & Owl Spring & VA-38 & $3 / 81$ & $\begin{array}{l}\text { SE-1/4, Sec } 7 \text {, } \\
\text { T16N, R2E }\end{array}$ & m & 16 & 7.22 & 25 & Spring flows from Madera limestone. & P-H-A; T78 (A8) \\
\hline
\end{tabular}

a Location no. on Fig. 2. 


\section{TABLE B-I (cont)}

b im surface meteoric

$\mathrm{cm}$ carbonated meteoric

a acid-sulphate

$d$ deep geothermal + derivative

connate

CG-G: Goff and Grigsby $^{1}$

$G$ et al.: Goff et al. ${ }^{2}$

G-S: Goff and Sayer

Gr: Griggs ${ }^{14}$

M: Mariner et al. 26

P77: Purtymun 11

P79: Purtymun 27

P-C: Purtymun and Cooper 28

P-P-0: Purtymun et al. ${ }^{10}$

P-H-A: Purtymun et al. 9

S: Summers 24

T-74: Trainer 5

T-75: Trainer

T-78: Trainer ${ }^{29}$; Trainer's sample no. given in parentheses.

Water samples collected for isotopes and for chemical analysis at different times. 
TABLE B-II

MAJOR ELEMENT ANALYSES FOR WATERS IN THE JEMEZ MOUNTAINS REGION, NEW MEXICO (VALUES IN mg/l)

\begin{tabular}{|c|c|c|c|c|c|c|c|c|c|c|c|c|c|c|c|c|c|c|c|}
\hline $\begin{array}{l}\text { Map } \\
\text { No. }\end{array}$ & Name & $\begin{array}{l}\text { Field } \\
\text { No. }\end{array}$ & ${ }_{\text {Temp. }}^{\text {Temp. }}$ & $\begin{array}{c}\text { Field } \mathrm{C} \\
\mathrm{pH}\end{array}$ & $\begin{array}{c}\text { Conductivity } \\
\text { umhos } / \mathrm{cm}\end{array}$ & $\mathrm{SiO}_{2}$ & A) & $\mathrm{Fe}$ & Mn & $\mathrm{Ca}$ & $\mathrm{Mg}$ & $\mathrm{Na}$ & $k$ & Li & $\mathrm{HCO}_{3}$ & $\mathrm{SO}_{4}$ & $\mathrm{Cl}$ & $\mathbf{F}$ & B \\
\hline \multicolumn{20}{|c|}{ Los Alamos Area, Pajarito Plateau } \\
\hline 1 & Gallery Spring & $L A-1$ & 11 & 5.6 & - & 43 & -- & $<0.04$ & $<0.02$ & 7.0 & 3.3 & 5.8 & 1.4 & 0.02 & 52 & $<5$ & $<1$ & 0.12 & $<0.05$ \\
\hline 2 & T-3 Hell & LA-2 & 13 & 6.5 & $\cdots$ & 15 & -- & 0.53 & 0.11 & 14.0 & 5.0 & 11.0 & 1.9 & 0.03 & 102 & 5 & 4 & 0.26 & $<0.05$ \\
\hline 3 & T-2 Well & LA-3 & 11 & 5.7 & - & 5 & -- & $<0.04$ & $<0.02$ & 11.0 & 2.7 & 8.8 & 0.88 & 0.03 & 78 & 5 & 2 & 0.46 & $<0.05$ \\
\hline 4 & Sacred Spring & LA-4 & 14 & 5.7 & -- & 34 & -- & $<0.04$ & $<0.02$ & 22 & 0.45 & 20 & 2.5 & 0.04 & 114 & 7 & 2 & 0.46 & $<0.05$ \\
\hline 5 & Basalt Spring & LA-5 & 15 & 5.8 & -- & 44 & -- & $<0.04$ & $<0.02$ & 26 & 7.6 & 12 & 3.1 & 0.03 & 98 & 18 & 12 & 0.32 & $<0.05$ \\
\hline 6 & L-6 Well & LA-6 & 27 & 6.8 & -- & 33 & -- & $<0.04$ & $<0.02$ & 2.8 & 0.15 & 72 & 0.8 & 0.04 & 170 & 6 & 4 & 2.2 & $<0.05$ \\
\hline 71 & L-1B Well & LA-7 & 30 & 7.2 & -- & 36 & -- & $<0.04$ & $<0.02$ & 6.5 & 0.30 & 138 & 2.0 & 0.11 & 326 & 32 & 15 & 2.3 & 0.45 \\
\hline 8 & L-5 Well & LA-8 & 26.5 & 6.5 & - & 40 & -- & $<0.04$ & $<0.02$ & 7.2 & 0.13 & 52 & 1.3 & 0.04 & 143 & 6 & 3 & 0.98 & $<0.05$ \\
\hline 9 & L-4 Well & LA-9 & 28 & 6.5 & -- & 39 & -- & $<0.04$ & $<0.02$ & 10 & 0.22 & 21 & 1.7 & 0.03 & 85 & 5 & 4 & 0.33 & 0.38 \\
\hline 10 & PM-2 Well & LA-10 & 23.5 & 6.5 & -- & 83 & -- & $<0.04$ & $<0.02$ & 8.8 & 3.0 & 9.6 & 1.7 & 0.02 & 65 & $<5$ & 3 & 0.19 & 0.25 \\
\hline 11 & PM-1 Well & LA-11 & 28 & 6.5 & -- & 82 & -- & $<0.04$ & $<0.02$ & 26 & 6.8 & 18 & 3.6 & 0.03 & 146 & 6 & 6 & 0.26 & 0.25 \\
\hline 12 & G-6 Well & LA-12 & 30.5 & 6.5 & -- & 55 & -- & $<0.04$ & $<0.02$ & 15 & 2.3 & 15 & 2.0 & $<0.02$ & 94 & 5 & 2 & 0.27 & $<0.05$ \\
\hline 13 & G-5 Well & LA-13 & 26.5 & 6.5 & -- & 59 & $\cdots$ & $<0.04$ & $<0.02$ & 17 & 3.9 & 11 & 1.8 & $<0.02$ & 93 & 5 & 2 & 0.25 & 0.12 \\
\hline 14 & G-4 Hell & $L A-14$ & 26 & 6.5 & - & 53 & -- & $<0.04$ & $<0.02$ & 16 & 2.5 & 14 & 1.8 & $<0.02$ & 92 & 5 & 2 & 0.27 & 0.12 \\
\hline 15 & G-3 Well & LA-15 & 29 & 6.5 & -- & 59 & -- & $<0.04$ & $<0.02$ & 11 & 1.2 & 22 & 1.6 & $<0.02$ & 93 & 5 & 2 & 0.45 & $<0.05$ \\
\hline 16 & G-2 Well & LA-16 & 30 & 6.5 & -- & 77 & -- & $<0.04$ & $<0.02$ & 11 & 0.61 & 33 & 2.5 & 0.02 & 122 & 5 & 4 & 1.0 & 0.12 \\
\hline 17 & G-1A Well & LA-17 & 28 & 6.5 & -- & 78 & -- & $<0.04$ & $<0.02$ & 11 & 0.58 & 24 & 2.8 & $<0.02$ & 100 & 5 & 1 & 0.55 & $<0.05$ \\
\hline 18 & G-1 Well & LA-18 & 26 & 6.5 & -- & 84 & -- & $<0.04$ & $<0.02$ & 13 & 0.68 & 22 & 3.1 & $<0.02$ & 97 & 5 & 1 & 0.50 & $<0.05$ \\
\hline 19 & $\begin{array}{l}\text { Spring, White } \\
\text { Rock Canyon }\end{array}$ & LA-19. & 19 & 6.5 & -- & 71 & -- & $<0.04$ & $<0.02$ & 12 & 3.1 & 11 & 1.4 & 0.03 & 74 & $<5$ & $<1$ & 0.45 & $<0.05$ \\
\hline 20 & PM-3 Kell & LA-20 & 27.5 & 6.5 & -- & 91 & -- & $<0.04$ & $<0.02$ & 26 & 8.7 & 16 & 3.3 & 0.04 & 146 & 6 & 12 & 0.28 & 0.18 \\
\hline
\end{tabular}


TABLE B-II (cont)

\begin{tabular}{|c|c|c|c|c|c|c|c|c|c|c|c|c|c|c|c|c|c|c|c|}
\hline $\begin{array}{l}\text { Map } \\
\text { No. }\end{array}$ & Name & $\begin{array}{l}\text { Fle?d } \\
\text { No. }\end{array}$ & $\operatorname{Temp}_{{ }^{-}}$ & $\begin{array}{c}\text { Fleld } \\
\text { PH }\end{array}$ & $\begin{array}{c}\text { Conductivity } \\
\text { umhos } / \mathrm{cm}\end{array}$ & $\mathrm{SiO}_{2}$ & Al & $\mathrm{Fe}$ & 的 & $\mathrm{Ca}$ & Mg & $\mathrm{Na}$ & k & Li & $\mathrm{HCO}_{3}$ & $\mathrm{SO}_{4}$ & Cl & $\mathbf{F}$ & B \\
\hline \multicolumn{4}{|c|}{ Valles Caldera, Sulphur Springs Area } & & & & & $r$ & & & & & & & & & & & \\
\hline 21 & $\begin{array}{l}\text { Mudpot, Men's } \\
\text { Bathhouse }\end{array}$ & VA-13 & 78 & 2.52 & 4050 & 103 & -- & 13 & 0.01 & 2.1 & 1.25 & 2.1 & 8.2 & 0.02 & 0 & 786 & 2.48 & 6.36 & $<0.1$ \\
\hline 21 & $\begin{array}{l}\text { Mudpot, Men's } \\
\text { Bathhouse }\end{array}$ & $\$-7-80$ & 82 & 2.00 & 10300 & 246 & - & 37 & 0.64 & 10 & 6.5 & 6.0 & 35 & 0.04 & 0 & 2500 & $<1$ & $<0.20$ & 0.1 \\
\hline 21. & Unnamed Hot Spg. & VA-14 & 63 & 2.38 & 5800 & 230 & 3.17 & 18.1 & 11.7 & 90.8 & 16.2 & 14.6 & 18.7 & 0.05 & 0 & 2110 & 3.72 & 0.61 & $<0.1$ \\
\hline 21 & Unnamed Hot Spg. & $S-9-80$ & - & 2.03 & 5700 & 243 & -- & 38 & 3.20 & 165 & 40.0 & 16.4 & 6.4 & 0.09 & 0 & 1750 & 8.3 & 1.75 & $<0.03$ \\
\hline 21 & Lemonade Spring & $S-10-80$ & 58 & 2.30 & -- & 238 & -- & 38 & 3.76 & 168 & 42 & 7.7 & 5.6 & 0.14 & 0 & 2740 & 17 & 0.52 & 0.03 \\
\hline 21 & Unnamed Spring & $5-3-80$ & 11 & 3.60 & 1910 & 59 & -- & 16.2 & 2.84 & 280 & 33 & 25 & 27 & 0.29 & 0 & 1500 & 20 & 0.70 & 0.2 \\
\hline 21 & Footbath Spring & $S-4-80$ & 33 & 1.10 & 30200 & 214 & -- & 468 & 4.65 & 56 & 26.5 & 10.8 & 94 & 0.10 & 0 & 7900 & $<1$ & 10.6 & 0.2 \\
\hline 21 & Electric Spring & $S-5-80$ & 36 & 1.50 & 12800 & 228 & -- & 127 & 2.40 & 114 & 23.0 & 8.5 & 66 & 0.06 & 0 & 4100 & $<1$ & 5.2 & $<0.1$ \\
\hline 21 & Women's Bathhouse & $S-6-80$ & $90^{\circ}$ & 1.40 & 13400 & 168 & -- & 490 & 8.10 & 131 & 50.0 & 18.9 & 72 & 0.17 & 0 & 6400 & $<1$ & 5.2 & 0.2 \\
\hline 22 & $\begin{array}{l}\text { Spring, Al amo } \\
\text { Canyon }\end{array}$ & $5-1-80$ & 11 & 4.20 & .430 & 53 & -- & 16.3 & 0.44 & 15.2 & 2.49 & 13.0 & 6.2 & 0.06 & 0 & 113 & 11 & 0.52 & $<0.1$ \\
\hline 22 & $\begin{array}{l}\text { Creek, Alamo } \\
\text { Canyon }\end{array}$ & $S-2-80$ & 11 & 3.10 & 340 & 41 & - & 1.1 & 0.35 & 12.8 & 2.29 & 14.1 & 6.3 & 0.06 & 0 & 101 & 13 & $<0.20$ & $<0.1$ \\
\hline 23 & Bubbling Pool & $V A-22$ & 0.5 & 4.5 & 280 & 44 & -- & 1.37 & 0.30 & 14.1 & 2.75 & 5.8 & 4.5 & 0.4 & 0 & 109 & 4.9 & 0.23 & $<0.1$ \\
\hline 22 & Bubbling Seep & $v A-23$ & 7 & 5.2 & 730 & 51 & - & 0.28 & 0.96 & 83.5 & 12.0 & 32.8 & 7.9 & 0.08 & 178 & 254 & 7.2 & 0.23 & $<0.1$ \\
\hline 24 & $\begin{array}{l}\text { Spring, Short } \\
\text { Canyon }\end{array}$ & $5-8-80$ & 8 & 4.10 & 500 & 55 & -- & 5.5 & 0.54 & 43 & 5.9 & 7.8 & 7.3 & 0.02 & 0 & 199 & 5.9 & $<0.20$ & $<0.1$ \\
\hline \multicolumn{20}{|c|}{ Valles Caldera, Thermal Meteoric (Ring Fracture Zone) } \\
\hline 25 & Spence Hot Spring & VA-1 & 45 & 6.7 & -- & 66 & -- & $<0.04$ & $<0.02$ & 5.5 & 1.9 & 50 & 1.3 & 0.66 & 144 & 16 & 8 & 0.55 & 0.15 \\
\hline 25 & $\begin{array}{l}\text { Little Spence } \\
\text { Hot Spring }\end{array}$ & VA-2 & 34 & 6.7 & -- & 67 & -- & $<0.04$ & $<0.02$ & 8.8 & 1.9 & 56 & 1.5 & 0.66 & 152 & 25 & 7 & 0.70 & 0.13 \\
\hline 26 & McCauley Spring & VA-3 & 31 & 6.2 & -- & 56 & - & $<0.04$ & $<0.02$ & 8.5 & 4.9 & 18 & 0.8 & 0.24 & 86 & 7 & 6 & 0.85 & 0.24 \\
\hline 27 & $\begin{array}{l}\text { San Antonio Hot } \\
\text { Spring }\end{array}$ & $v_{n-4}$ & 42 & 6.8 & -- & 79 & -- & $<0.04$ & 0.02 & 2.3 & 0.30 & 21 & 1.7 & $<0.02$ & 56 & 7 & 2 & 0.80 & $<0.05$ \\
\hline 28 & Bathhouse Spring & $V A-20$ & 38 & 6.1 & 163 & 96 & 0.041 & $<0.08$ & $<0.04$ & 4.98 & 0.40 & 24.5 & 3.7 & 0.06 & 71 & 15 & 2.4 & 1.6 & $<0.1$ \\
\hline
\end{tabular}




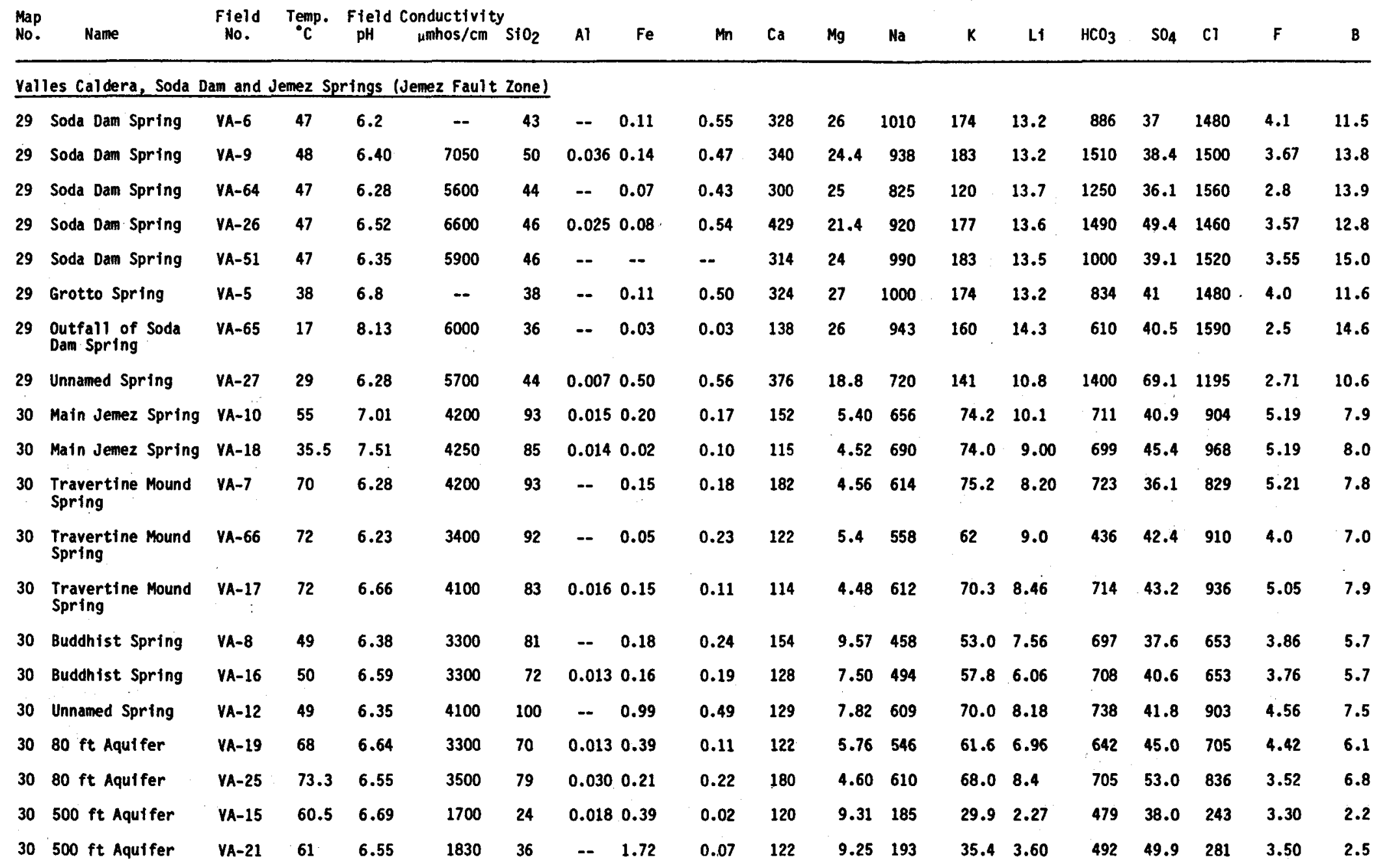


TABLE B-II (cont)

\begin{tabular}{|c|c|c|c|c|c|c|c|c|c|c|c|c|c|c|c|c|c|c|c|}
\hline $\begin{array}{l}\text { Map } \\
\text { No. }\end{array}$ & Name. & $\begin{array}{l}\text { Fleld } \\
\text { No. }\end{array}$ & Temp. & $\begin{array}{c}\text { Field } 0 \\
\mathrm{pH}\end{array}$ & $\begin{array}{c}\text { Conductivity } \\
\mu \text { mhos } / \mathrm{cm}\end{array}$ & $\mathrm{SiO}_{2}$ & A) & $\mathrm{Fe}$ & $M n$ & $\mathrm{Ca}$ & $\mathrm{Mg}$ & $\mathrm{Na}$ & K & Li & $\mathrm{HCO}_{3}$ & $\mathrm{SO}_{4}$ & $\mathrm{Cl}$ & $\mathbf{F}$ & B \\
\hline \multicolumn{2}{|c|}{ Valles Caldera Region, } & Miscell & aneous & & & & & & & & & & & & & & & & \\
\hline 28 . & San Antonio Creek & VA-24 & 2 & -- & -- & -- & $-\infty$ & -- & -- & -- & -- & -- & -- & -- & -- & -- & -- & -- & -- \\
\hline 31 . & Panorama Spring & YA-28 & 13 & 7.67 & 920 & 62 & 0.0013 & 0.016 & 0.003 & 101 & 22.9 & 114 & 5.3 & 0.16 & 519 & 56.7 & 21.7 & 0.97 & $<0.1$ \\
\hline 32 & Pajarito Spring & VA-29 & 20 & 5.9 & 210 & 67 & -- & 0.044 & 0.002 & 19.6 & 5.3 & 11.8 & 2.08 & 0.08 & 100 & 7.5 & 6.4 & 0.46 & 0.05 \\
\hline 33 & $\begin{array}{l}\text { Spring, White } \\
\text { Rock Canyon. }\end{array}$ & $V A-30$ & 18 & 6.0 & -- & 63 & - & 0.140 & 0.009 & 29.3 & 6.9 & 14.4 & 2.4 & 0.07 & -- & 8.8 & 9.8 & 0.48 & 0.07 \\
\hline 34 & Cold Mineral Seep & VA-31 & 19 & 8.37 & 4200 & 18 & 0.096 & 0.005 & $<0.001$ & 10.2 & 71.0 & 613 & 42.0 & 3.22 & 1745 & 3732 & 284 & 4.95 & 4.15 \\
\hline 35 & Sino Spring & VA-32 & 21 & 7.45 & -- & -- & - & -- & -- & -- & -- & -- & -- & - & -- & -- & - & - & -- \\
\hline 35 & Sino Spring & VA-63 & 18 & 7.10 & 163 & 78 & 0.001 & $<0.001$ & $<0.001$ & 11.2 & 3.6 & 14 & 0.2 & 0.12 & 82 & 5.0 & 6.0 & 0.32 & 0.01 \\
\hline 36 & Unnamed Spring & VA-39 & 11 & 6.91 & 2500 & 16 & 0.021 & 0.272 & 0.144 & 49 & 10.3 & 447. & 34 & 2.2 & 848 & 193 & 267 & 7.2 & 3.16 \\
\hline 37 & Canon -Spring & $V A-40$ & 18 & 7.58 & 1090 & 47 & 0.006 & 0.81 & 0.148 & 36 & 5.2 & 161 & 7.6 & 0.5 & 367 & 127 & 71 & 9.3 & 0.41 \\
\hline 38 & $\begin{array}{l}\text { Indian Valley } \\
\text { Well }\end{array}$ & $V A-41$ & 17.5 & 6.81 & 280 & 77 & -- & 0.42 & 0.024 & 23.5 & 6.2 & 22.0 & 4.78 & 0.044 & 137 & 6.2 & 7.3 & 0.17 & $<0.003$ \\
\hline 39 & $\begin{array}{l}\text { Unnamed Cold } \\
\text { Spring }\end{array}$ & $V A-42$ & 15 & 7.20 & 88 & 52 & - & 0.006 & $<0.001$ & 6.4 & 1.66 & 1.7 & 8.0 & 0.030 & 34 & 2.6 & 9.1 & 0.39 & $<0.01$ \\
\hline 40 & $\begin{array}{l}\text { Unnamed cold } \\
\text { Spring }\end{array}$ & $V_{A-43}$ & 15 & 6.68 & 65 & 45 & -- & 0.014 & 0.006 & 5.3 & 1.03 & 3.8 & 3.8 & 0.007 & 11 & 13.1 & 6.9 & 0.33 & $<0.01$ \\
\hline 41 & $\begin{array}{l}\text { Unnamed cold } \\
\text { Spring }\end{array}$ & VA-44 & 8 & 6.91 & 210 & 48 & -- & 0.025 & 0.002 & 19.0 & 5.0 & 10.6 & 9.08 & 0.002 & 90 & 17.4 & 7.4 & 0.11 & $<0.003$ \\
\hline 42 & Horseshoe Spring & $V A-45$ & 12 & 6.89 & 240 & 46 & $=-$ & $<0.004$ & $<0.001$ & 19.0 & 3.4 & 30.7 & 3.00 & 0.088 & 145 & 6.2 & 6.1 & 0.21 & 0.003 \\
\hline 43 & $\begin{array}{l}\text { Unnaned cold } \\
\text { Spring }\end{array}$ & VA-46 & 10 & 6.42 & 126 & 74 & -- & 0.004 & $<0.001$ & 10.7 & 1.50 & 11.6 & 2.53 & 0.040 & 57 & 4.4 & 3.5 & 0.44 & $<0.003$ \\
\hline 44 & $\begin{array}{l}\text { Unnamed cold } \\
\text { Spring }\end{array}$ & VA-47 & 10 & 6.48 & 115 & 41 & -- & 0.036 & 0.001 & 12.3 & 1.54 & 7.23 & 2.10 & 0.018 & 49 & 8.7 & 3.6 & 0.21 & $<0.003$ \\
\hline 45 & $\begin{array}{l}\text { Unnamed Cold } \\
\text { Spring }\end{array}$ & VA-48 & 9 & 5.6 & 280 & 22 & - & 0.036 & $<0.001$ & 33 & 8.7 & 5.9 & 2.1 & 0.003 & 59 & 78 & 6.2 & 0.28 & $<0.01$ \\
\hline 46 & Eddy's Well & VA-49 & 15 & 7.24 & 550 & 64 & -- & 0.029 & $<0.001$ & 56 & 13.8 & 18 & 9.6 & 0.098 & 332 & 28.6 & 8.0 & 0.52 & $<0.01$ \\
\hline 34 & $\begin{array}{l}\text { Cold Mineral } \\
\text { Seep }\end{array}$ & YA-50 & 11 & 7.92 & 4200 & 17 & -- & 0.004 & $<0.001$ & 35 & 46 & 940 & 47 & 4.0 & 1980 & 290 & 323 & 5.75 & 4.47 \\
\hline
\end{tabular}




\begin{tabular}{|c|c|c|c|c|c|c|c|c|c|c|c|c|c|c|c|c|c|c|c|}
\hline $\begin{array}{l}\text { Map } \\
\text { No. }\end{array}$ & Name & $\begin{array}{l}\text { Field } \\
\text { No. }\end{array}$ & ${ }^{\text {Temp. }}$ & $\underset{\mathrm{pH}}{\text { Fleld C }}$ & $\begin{array}{l}\text { Conductivit } \\
\mu \text { mhos } / \mathrm{cm}\end{array}$ & $\mathrm{SiO}_{2}$ & A1 & $\mathrm{Fe}$ & $M_{n}$ & $\mathrm{Ca}$ & Mg & $\mathrm{Na}$ & k & LI & $\mathrm{HCO}_{3}$ & $\mathrm{SO}_{4}$ & CI & $F$ & B \\
\hline 29 & Jemez River & $V A-52$ & 5 & 5.30 & 100 & 17 & $\ldots$ & 0.360 & 0.014 & 11 & 1.4 & 5.6 & 2.0 & 0.040 & 52 & 9.0 & 4.0 & 0.56 & 0.09 \\
\hline 48 & $\begin{array}{l}\text { Unnamed Cold } \\
\text { Spring }\end{array}$ & VA-54 & 11 & 5.4 & 99 & 30 & - & 0.360 & 0.009 & 10.0 & 2.0 & 3.5 & 2.9 & 0.002 & 40 & 9.9 & 5.4 & 0.22 & $<0.01$ \\
\hline 49 & $\begin{array}{l}\text { Unnamed Cold } \\
\text { Spring }\end{array}$ & VA-55 & 8.5 & 5.4 & 55 & 34 & -- & 1.18 & 0.012 & 5.0 & 1.4 & 2.7 & 1.8 & 0.005 & 24 & 6.0 & 5.5 & 0.21 & $<0.01$ \\
\hline 50 & $\begin{array}{l}\text { Unnamed Cold } \\
\text { Spring }\end{array}$ & YA-56 & 6.5 & 5.4 & 105 & 38 & -- & 0.60 & 0.007 & 8.7 & 3.0 & 5.0 & 2.9 & 0.004 & 39 & 6.7 & 9.8 & 0.20 & $<0.01$ \\
\hline 51 & Apache Spring & YA-57 & 9 & 5.3 & 137 & 58 & -- & 0.42 & 0.003 & 10.8 & 4.6 & 6.7 & 3.5 & 0.006 & 57 & 8.3 & 8.0 & 0.27 & $<0.01$ \\
\hline 52 & $\begin{array}{l}\text { Unnamed Cold } \\
\text { Spring }\end{array}$ & VA-58 & 15 & 5.4 & 130 & 65 & - & 0.15 & 0.002 & 10.6 & 3.4 & 9.4 & 3.1 & 0.024 & 75 & 3.4 & 7.1 & 0.30 & $<0.01$ \\
\hline 53 & $\begin{array}{l}\text { Unnamed Cold } \\
\text { Spring }\end{array}$ & VA-59 & 17 & 5.6 & 130 & 69 & -- & 1.19 & 0.135 & 10.4 & 3.4 & 9.0 & 3.2 & 0.024 & 73 & 2.4 & 7.5 & 0.35 & $<0.01$ \\
\hline 54 & Turkey Springs & $Y A-60$ & 18 & 5.5 & 193 & 61 & -- & 0.012 & 0.001 & 20 & 5.5 & 10.1 & 1.7 & 0.010 & 103 & 4.2 & 8.0 & 0.38 & 0.03 \\
\hline 46 & Henson's Hell & VA-61 & 19 & 6.82 & 560 & 62 & -- & $<0.02$ & 0.003 & 59 & 8.4 & 36 & 4.6 & 0.136 & 305 & 20.5 & 7.7 & 0.38 & 0.01 \\
\hline \multicolumn{20}{|c|}{ San $Y_{s}$} \\
\hline 55 & $\begin{array}{l}\text { Harm Mineral } \\
\text { Spring }\end{array}$ & VA-33 & 27 & 6.57 & 11550 & 14 & 0.014 & 3.40 & 1.80 & 375 & 128 & 1710 & 75.3 & 5.30 & 1860 & 2330 & 1820 & 3.95 & 8.32 \\
\hline 56 & Zia Hot Hell & VA-34 & 56 & 6.29 & 16600 & 30 & 0.052 & 1.40 & 0.10 & 302 & 90 & 2650 & 66.7 & 6.70 & 1440 & 3740 & 3000 & 2.40 & 6.52 \\
\hline 56 & 21a Hot Well & VA-53 & 54 & 6.53 & 16000 & 33 & -- & $0.240 ?$ & 0.022 & 321 & 61 & 3440 & 77 & 6.0 & 1068 & 3430 & 3210 & 4.51 & 7.41 \\
\hline 56 & Zia Hot Nell & VA-67 & 53 & 6.72 & 15800 & 35 & 0.022 & 1.46 & 0.040 & 320 & 71.5 & 3180 & 64 & 6.3 & 1400 & 3280 & 2930 & 3.8 & 6.60 \\
\hline 57 & Unnamed Well & $V A-35$ & 21 & -- & -- & -- & -- & - & -- & -- & -- & -- & -- & -- & -- & -- & -- & -- & -- \\
\hline 58 & Salt Spring & VA-36 & 15.5 & 7.90 & 10500 & 1 & 0.005 & 0.030 & 0.024 & 89 & 26.4 & 1950 & 94 & 7.4 & 1870 & 702 & 2380 & 10.8 & 9.2 \\
\hline 59 & Log Spring & VA-37 & 28.5 & -- & -- & -- & -- & - & -- & -- & -- & -- & -- & -- & -- & -- & - & -- & -- \\
\hline 60 & Onl Spring & $V A-38$ & 16 & 7.22 & 620 & 22 & 0.001 & $<0.001$ & $<0.001$ & 73 & 10.4 & 34 & 2.1 & 0.176 & 311 & 33.8 & 8 27.8 & 1.4 & 0.12 \\
\hline
\end{tabular}




\begin{tabular}{|c|c|c|c|c|c|c|c|c|c|c|c|c|c|}
\hline $\begin{array}{l}\text { Map } \\
\text { No. }\end{array}$ & Name & $\begin{array}{l}\text { Fleld } \\
\text { Ho. }\end{array}$ & $\mathrm{Ag}$ & $\mathbf{B a}$ & cd & Co & $\mathrm{Cr}$ & $\mathrm{Cu}$ & mo & Mi & Pb & $s r$ & Zn \\
\hline \multicolumn{14}{|c|}{ Los Alamos Area, Pajarito Plateau } \\
\hline 1 & Gallery Spring & LA-1 & $<0.03$ & $<0.12$ & $<0.03$ & $<0.06$ & $<0.03$ & $<0.04$ & $<0.10$ & $<0.05$ & $<0.14$ & 0.05 & $<0.01$ \\
\hline 2 & T-3 Well & LA-2 & $<0.03$ & $<0.12$ & $<0.03$ & $<0.06$ & $<0.03$ & $<0.04$ & $<0.10$ & $<0.05$ & $<0.14$ & 0.05 & 0.01 \\
\hline 3 & T-2 Mell & LA-3 & $<0.03$ & $<0.12$ & $<0.03$ & $<0.06$ & $<0.03$ & $<0.04$ & $<0.10$ & $<0.05$ & $<0.14$ & 0.03 & 0.18 \\
\hline 4 & Sacred Spring & $L A-4$ & 0.06 & $<0.12$ & 0.03 & $<0.06$ & $<0.03$ & $<0.04$ & $<0.10$ & $<0.05$ & $<0.14$ & 0.42 & 0.02 \\
\hline 5 & Basalt Spring & LA-5 & $<0.03$ & $<0.12$ & $<0.03$ & $<0.06$ & $<0.03$ & $<0.04$ & $<0.10$ & $<0.05$ & $<0.14$ & 0.12 & $<0.01$ \\
\hline 6 & L-6 Mell & $L A-6$ & $<0.03$ & $<0.12$ & $<0.03$ & $<0.06$ & $<0.03$ & $<0.04$ & $<0.10$ & $<0.05$ & $<0.14$ & 0.05 & $<0.01$ \\
\hline 7 & L-1B Vell & LA-7 & $<0.03$ & $<0.12$ & $<0.03$ & $<0.06$ & $<0.03$ & $<0.04$ & $<0.10$ & $<0.05$ & $<0.14$ & 0.14 & $<0.01$ \\
\hline 8 & L-5 Hell & LA-8 & $<.05$ & $<0.12$ & $<0.03$ & $<0.06$ & $<0.03$ & $<0.04$ & $<0.10$ & $<0.05$ & $<0.14$ & 0.10 & $<0.01$ \\
\hline 9 & L-4 Mell & LA-9 & $<0.03$ & $<0.12$ & $<0.03$ & $<0.06$ & $<0.03$ & $<0.04$ & $<0.10$ & $<0.05$ & $<0.14$ & 0.07 & $<0.01$ \\
\hline 10 & PM-2 Mell & LA-10 & $<0.03$ & $<0.12$ & $<0.03$ & $<0.06$ & $<0.03$ & $<0.04$ & $<0.10$ & $<0.05$ & $<0.14$ & 0.04 & $<0.01$ \\
\hline 11 & PM-1 Nell & LA-11 & $<0.03$ & $<0.12$ & $<0.03$ & $<0.06$ & $<0.03$ & $<0.04$ & $<0.10$ & $<0.05$ & $<0.14$ & 0.14 & $<0.01$ \\
\hline 12 & G-6 Hell & $L A-12$ & $<0.03$ & $<0.12$ & $<0.03$ & $<0.06$ & $<0.03$ & $<0.04$ & $<0.10$ & $<0.05$ & $<0.14$ & 0.06 & $<0.01$ \\
\hline 13 & G-5 Well & $L A-13$ & $<0.03$ & $<0.12$ & $<0.03$ & $<0.06$ & $<0.03$ & $<0.04$ & $<0.10$ & $<0.05$ & $<0.14$ & 0.08 & $<0.01$ \\
\hline 14 & G-4 Mell & $L A-14$ & $<0.03$ & $<0.12$ & $<0.03$ & $<0.06$ & $<0.03$ & $<0.04$ & $<0.10$ & $<0.05$ & $<0.14$ & 0.07 & $<0.01$ \\
\hline 15 & G-3 Hell & $L A-15$ & $<0.03$ & $<0.12$ & $<0.03$ & $<0.06$ & $<0.03$ & $<0.04$ & $<0.10$ & $<0.05$ & $<0.14$ & 0.06 & $<0.01$ \\
\hline 16 & G-2 Well & $L A-16$ & $<0.03$ & $<0.12$ & $<0.03$ & $<0.06$ & $<0.03$ & $<0.04$ & $<0.10$ & $<0.05$ & $<0.14$ & 0.08 & $<0.01$ \\
\hline 17 & G-1A Well & LA-17 & $<0.03$ & $<0.12$ & $<0.03$ & $<0.06$ & $<0.03$ & $<0.04$ & $<0.10$ & $<0.05$ & $<0.14$ & 0.08 & $<0.01$ \\
\hline 18 & G-1 Mell & LA-18 & 0.06 & $<0.12$ & $<0.03$ & $<0.06$ & $<0.03$ & $<0.04$ & $<0.10$ & $<0.05$ & $<0.14$ & 0.08 & $<0.01$ \\
\hline 19 & Spring, White Rock Canyon & LA-19 & $<0.03$ & $<0.12$ & $<0.03$ & $<0.06$ & $<0.03$ & $<0.04$ & $<0.10$ & $<0.05$ & $<0.14$ & 0.05 & $<0.01$ \\
\hline 20 & PM-3 Nell & $L A-20$ & 0.06 & $<0.12$ & $<0.03$ & $<0.06$ & $<0.03$ & $<0.04$ & $<0.10$ & $<0.05$ & $<0.14$ & 0.12 & 0.02 \\
\hline \multicolumn{14}{|c|}{ Valles Caldera, Sulphur Springs Area } \\
\hline 21 & Mudpot, Men's Bathhouse & VA-13 & $<0.03$ & $<0.12$ & $<0.03$ & $<0.06$ & $<0.03$ & 0.63 & $<0.10$ & $<0.05$ & $<0.14$ & 0.03 & 0.16 \\
\hline 21 & Mudpot, Men's Bathhouse & $S-7-80$ & $<0.03$ & 0.083 & $<0.001$ & 0.059 & -- & 0.030 & 0.010 & --- & --- & 0.113 & --- \\
\hline
\end{tabular}




\begin{tabular}{|c|c|c|c|c|c|c|c|c|c|c|c|c|c|}
\hline $\begin{array}{l}\text { Map } \\
\text { No. }\end{array}$ & Name & $\begin{array}{l}\text { Field } \\
\text { No. }\end{array}$ & $\mathrm{Ag}$ & $\mathrm{Ba}$ & cd & Co & $\mathrm{Cr}$ & $\mathrm{Cu}$ & Mo & Ni & $\mathrm{Pb}$ & $\mathrm{Sr}$ & $2 n$ \\
\hline 21 & Unnamed Hot Spring & VA-14 & $<0.03$ & $<0.12$ & 0.03 & 0.07 & 0.06 & $<0.04$ & $<0.10$ & $<0.05$ & $<0.14$ & 0.22 & 0.08 \\
\hline 21 & Unnamed Hot Spring & $S-9-80$ & $<0.03$ & 0.037 & $<0.001$ & 0.017 & 0.063 & $\cdots$ & $<0.002$ & 0.042 & 0.120 & 0.027 & --- \\
\hline 21 & Lemonade Spring & $S-10-80$ & $<0.03$ & 0.055 & $<0.001$ & 0.010 & 0.056 & $<0.001$ & $<0.002$ & 0.016 & 0.004 & 0.060 & 0.440 \\
\hline 21 & Unnamed Spring & $S-3-80$ & $<0.03$ & 0.010 & $<0.001$ & 0.024 & 0.052 & $<0.002$ & $<0.002$ & 0.039 & 0.032 & 0.610 & $\cdots$ \\
\hline 21 & Footbath Spring & $S-4-80$ & $<0.03$ & 0.030 & 0.004 & 0.460 & -- & --- & 0.005 & --- & --- & 0.098 & $\cdots$ \\
\hline 21 & Electric Spring & $S-5-80$ & $<0.03$ & 0.035 & $<0.001$ & 0.100 & 0.360 & 0.080 & 0.004 & 0.220 & 0.080 & 0.140 & 0.640 \\
\hline 21 & Women's Bathhouse & $S-6-80$ & $<0.03$ & 0.053 & 0.001 & 0.245 & -- & $\cdots$ & 0.010 & $\cdots$ & --- & 0.065 & --- \\
\hline 22 & Spring, Al amo Canyon & $S-1-80$ & $<0.03$ & 0.050 & $<0.001$ & $<0.001$ & 0.019 & 0.001 & $<0.002$ & 0.008 & 0.004 & 0.180 & 0.880 \\
\hline 22 & Creek, Alamo Canyon & $S-2-80$ & $<0.03$ & 0.042 & $<0.001$ & 0.002 & 0.005 & 0.008 & $<0.002$ & 0.003 & 0.004 & 0.140 & 0.560 \\
\hline 23 & Bubbling Pool & VA-22 & $<0.03$ & $<0.12$ & $<0.03$ & $<0.06$ & $<0.03$ & $<0.04$ & $<0.10$ & $<0.05$ & $<0.14$ & 0.40 & $<0.01$ \\
\hline 23 & Bubbling Seep & VA-23 & $<0.03$ & $<0.12$ & $<0.03$ & $<0.06$ & $<0.03$ & $<0.04$ & $<0.10$ & $<0.05$ & $<0.14$ & 0.68 & $<0.01$ \\
\hline 24 & Spring, Short Canyon & $5-8-80$ & $<0.03$ & 0.109 & $<0.001$ & 0.005 & 0.010 & 0.007 & $<0.002$ & 0.001 & $<0.004$ & 0.340 & 0.160 \\
\hline
\end{tabular}

Valles Caldera, Thermal Meteoric (Ring Fracture Zone)

\begin{tabular}{|c|c|c|c|c|c|c|c|c|c|c|c|c|c|}
\hline 25 & Spence Hot Spring & VA-1 & $<0.03$ & $<0.12$ & $<0.03$ & $<0.06$ & $<0.03$ & $<0.04$ & $<0.10$ & $<0.05$ & $<0.14$ & 0.03 & 0.02 \\
\hline 25 & Little Spence Hot Spring & VA-2 & $<0.03$ & $<0.12$ & $<0.03$ & $<0.06$ & $<0.03$ & $<0.04$ & $<0.10$ & $<0.05$ & $<0.14$ & 0.04 & 0.02 \\
\hline 26 & McCauley Spring & VA-3 & $<0.03$ & $<0.12$ & $<0.03$ & $<0.06$ & $<0.03$ & $<0.04$ & $<0.10$ & $<0.05$ & $<0.14$ & 0.02 & $<0.01$ \\
\hline 27 & San Antonio Hot Spring & VA-4 & $<0.03$ & $<0.12$ & $<0.03$ & $<0.06$ & $<0.03$ & $<0.04$ & $<0.10$ & $<0.05$ & $<0.14$ & $<0.02$ & $<0.01$ \\
\hline 28 & Bathhouse Spring & VA-20 & $<0.03$ & $<0.12$ & $<0.03$ & $<0.06$ & $<0.03$ & $<0.04$ & $<0.10$ & $<0.05$ & $<0.14$ & 0.02 & $<0.01$ \\
\hline
\end{tabular}

Valles Caldera, Soda Dam and Jemez Springs (Jemez Fault Zone)

\begin{tabular}{|c|c|c|c|c|c|c|c|c|c|c|c|c|c|}
\hline 29 & Soda Dam Spring & $V A-6$ & $<0.03$ & $<0.12$ & $<0.03$ & $<0.06$ & $<0.03$ & $<0.04$ & $<0.10$ & $<0.05$ & $<0.14$ & 1.38 & 0.02 \\
\hline 29 & Soda Dam Spring & VA-9 & $<0.03$ & $<0.12$ & 0.06 & $<0.06$ & $<0.03$ & $<0.04$ & $<0.10$ & 0.05 & $<0.14$ & 1.50 & 0.04 \\
\hline
\end{tabular}


TABLE B-III (cont)

\begin{tabular}{|c|c|c|c|c|c|c|c|c|c|c|c|c|c|}
\hline $\begin{array}{l}\text { Map } \\
\text { No. }\end{array}$ & Name & $\begin{array}{l}\text { Fleld } \\
\text { No. }\end{array}$ & Ag & $\mathrm{Ba}$ & Cd & Co & $\mathrm{Cr}$ & $\mathrm{Cu}$ & Mo & Ni & $\mathrm{Pb}$ & $s r$ & Zn \\
\hline 29 & Soda Dam Spring & $V A-64$ & $<0.001$ & 0.430 & $<0.001$ & $<0.002$ & $<0.001$ & $<0.001$ & $<0.002$ & $<0.006$ & $<0.004$ & 1.48 & $<0.002$ \\
\hline 29 & Soda Dam Spring & VA-26 & 0.002 & 0.396 & 0.002 & $<0.06$ & 0.062 & 0.004 & $<0.10$ & $<0.05$ & 0.014 & 2.02 & $<0.01$ \\
\hline 29 & Soda Dam Spring & VA-51 & $<0.03$ & 0.206 & $<0.001$ & $<0.001$ & $<0.001$ & $<0.001$ & $<0.002$ & $<0.002$ & 0.044 & 0.890 & $<0.002$ \\
\hline 29 & Grotto Spring & $V A-5$ & $<0.03$ & $<0.12$ & $<0.03$ & 0.09 & $<0.03$ & $<0.04$ & $<0.10$ & $<0.05$ & $<0.14$ & 1.40 & 0.01 \\
\hline 29 & Outfall from Soda Dam Spring & $V A-65$ & $<0.001$ & 0.360 & $<0.001$ & $<0.002$ & $<0.001$ & $<0.001$ & $<0.002$ & $<0.001$ & $<0.004$ & 1.27 & $<0.002$ \\
\hline 29 & Unnamed Spring & VA-27 & 0.002 & 0.206 & 0.002 & 0.002 & 0.098 & 0.006 & $<0.10$ & $<0.05$ & 0.006 & 1.90 & $<0.01$ \\
\hline 30 & Main Jemez Spring & $V A-10$ & $<0.03$ & $<0.12$ & 0.03 & $<0.06$ & $<0.03$ & $<0.04$ & $<0.10$ & 0.10 & $<0.14$ & 0.56 & 0.03 \\
\hline 30 & Main Jemez Spring & VA-18 & $<0.03$ & 0.24 & $<0.03$ & $<0.06$ & $<0.03$ & $<0.04$ & $<0.10$ & $<0.05$ & $<0.14$ & 0.60 & $<0.01$ \\
\hline 30 & Travertine Mound Spring & VA-7 & $<0.03$ & $<0.12$ & 0.03 & $<0.06$ & $<0.03$ & $<0.04$ & $<0.10$ & $<0.05$ & $<0.14$ & 0.60 & 0.03 \\
\hline 30 & Travertine Mound Spring & YA-66 & $<0.001$ & 0.232 & $<0.001$ & $<0.002$ & 0.001 & $<0.001$ & $<0.002$ & 0.002 & $<0.004$ & 0.59 & $<0.002$ \\
\hline 30 & Travertine Mound Spring & VA-17 & $<0.03$ & 0.20 & $<0.03$ & 0.06 & $<0.03$ & $<0.04$ & $<0.10$ & 0.10 & $<0.14$ & 0.54 & 0.02 \\
\hline 30 & Buddhist Spring & VA-8 & $<0.03$ & 0.60 & 0.03 & $<0.06$ & $<0.03$ & $<0.04$ & $<0.10$ & 0.10 & $<0.14$ & 0.56 & 0.02 \\
\hline 30 & Buddhist Spring & YA-16 & $<0.03$ & $<0.12$ & $<0.03$ & $<0.06$ & $<0.03$ & $<0.04$ & $<0.10$ & 0.10 & $<0.14$ & 0.52 & 0.04 \\
\hline 30 & Unnamed Spring & $V A-12$ & $<0.03$ & 0.35 & 0.06 & 0.12 & $<0.03$ & $<0.04$ & $<0.10$ & 0.10 & $<0.14$ & 0.64 & 0.02 \\
\hline 30 & $80 \mathrm{ft}$ Aquifer & VA-19 & $<0.03$ & $<0.12$ & $<0.03$ & $<0.06$ & $<0.03$ & $<0.04$ & $<0.10$ & $<0.05$ & $<0.14$ & 0.54 & $<0.01$ \\
\hline 30 & 80 ft Aquifer & $V A-25$ & 0.002 & 0.202 & $<0.03$ & 0.002 & 0.082 & 0.002 & $<0.10$ & $<0.05$ & 0.006 & 0.86 & $<0.01$ \\
\hline 30 & $500 \mathrm{ft}$ Aquifer & VA-15 & $<0.03$ & $<0.12$ & $<0.03$ & 0.10 & $<0.03$ & $<0.04$ & $<0.10$ & 0.10 & $<0.14$ & 0.40 & $<0.02$ \\
\hline 30 & $500 \mathrm{ft}$ Aquifer & VA-21 & $<0.03$ & $<0.12$ & $<0.03$ & $<0.06$ & $<0.03$ & $<0.04$ & $<0.10$ & $<0.05$ & $<0.14$ & 0.40 & $<0.01$ \\
\hline
\end{tabular}

Valles Caldera Region, Miscellaneous

\begin{tabular}{|c|c|c|c|c|c|c|c|c|c|c|c|c|c|}
\hline 28 & San Antonio Creek & VA-24 & $\cdots$ & -- & -- & -- & -- & -- & -- & -- & $\because$ & -- & -- \\
\hline 31 & Panorama Spring & VA-28 & 0.002 & 0.176 & $<0.03$ & $<0.06$ & 0.018 & 0.002 & $<0.10$ & 0.002 & 0.002 & 0.720 & $<0.01$ \\
\hline 32 & Pajarito Spring & VA-29 & $<0.03$ & 0.010 & $<0.03$ & $<0.06$ & 0.018 & $<0.04$ & $<0.10$ & $<0.05$ & $<0.14$ & 0.132 & 0.001 \\
\hline 3 & Spring, White Rock Canyon & $V A-30$ & $<0.03$ & 0.014 & $<0.03$ & $<0.06$ & 0.006 & 0.004 & $<0.10$ & 0.004 & $<0.14$ & 0.225 & 0.003 \\
\hline
\end{tabular}


TABLE B-III (cont)

\begin{tabular}{|c|c|c|c|c|c|c|c|c|c|c|c|c|c|}
\hline $\begin{array}{l}\text { Map } \\
\text { No. }\end{array}$ & Name & $\begin{array}{l}\text { Field } \\
\text { No. }\end{array}$ & Ag & $\mathrm{Ba}$ & cd & Co & $\mathrm{Cr}$ & $\mathrm{Cu}$ & Mo & Ni & $\mathrm{Pb}$ & Sr & $2 n$ \\
\hline 34 & Cold Mineral Seep & $V A-31$ & 0.005 & 0.062 & 0.002 & $<0.06$ & $<0.03$ & $<0.04$ & 0.002 & $<0.05$ & $<0.14$ & 0.146 & $<0.01$ \\
\hline 35 & Sino Spring & VA-32 & -- & -- & -- & -- & -- & -- & - & -- & -- & -- & -- \\
\hline 35 & Sino Spring & VA-63 & $<0.001$ & 0.017 & $<0.001$ & $<0.002$ & $<0.001$ & $<0.001$ & $<0.002$ & $<0.001$ & $<0.004$ & 0.06 & 0.007 \\
\hline 36 & Unnamed Spring & YA-39 & $<0.001$ & 0.044 & $<0.001$ & $<0.002$ & $<0.001$ & $<0.001$ & 0.014 & $<0.001$ & $<0.004$ & 0.95 & $<0.002$ \\
\hline 37 & Canon Spring & VA-40 & $<0.001$ & 0.082 & $<0.001$ & $<0.002$ & $<0.05$ & $<0.05$ & 0.048 & $<0.03$ & $<0.004$ & 0.49 & 0.016 \\
\hline 38 & Indian Valley Well & VA-41 & $<0.03$ & 0.053 & $<0.03$ & 0.001 & 0.002 & $<0.04$ & 0.002 & $<0.05$ & $<0.14$ & 0.160 & 0.002 \\
\hline 39 & Unnamed Cold Spring & VA-42 & $<0.03$ & 0.010 & $<0.001$ & $<0.001$ & 0.002 & $<0.002$ & $<0.002$ & $<0.002$ & $<0.004$ & 0.030 & 0.025 \\
\hline 40 & Unnamed Cold Spring & VA-43 & $<0.03$ & 0.031 & $<0.001$ & $<0.001$ & $<0.001$ & 0.020 & $<0.002$ & $<0.002$ & $<0.004$ & 0.038 & 0.060 \\
\hline 41 & Unnamed Cold Spring & VA-44 & $<0.03$ & 0.129 & $<0.03$ & $<0.06$ & 0.001 & $<0.04$ & $<0.10$ & $<0.05$ & $<0.14$ & 0.120 & 0.006 \\
\hline 42 & Horseshoe Spring & $V A-45$ & $<0.03$ & 0.119 & $<0.03$ & 0.001 & 0.008 & $<0.04$ & 0.001 & $<0.05$ & $<0.14$ & 0.088 & $<0.01$ \\
\hline 43 & Unnamed Cold Spring & VA-46 & $<0.03$ & 0.047 & $<0.03$ & $<0.06$ & 0.001 & $<0.04$ & 0.001 & $<0.05$ & $<0.14$ & 0.072 & 0.008 \\
\hline 44 & Unnamed Cold Spring & VA-47 & $<0.03$ & 0.036 & $<0.03$ & $<0.06$ & 0.001 & $<0.04$ & 0.001 & $<0.05$ & $<0.14$ & 0.071 & $<0.01$ \\
\hline 45 & Unnamed Cold Spring & VA-48 & $<0.03$ & 0.068 & $<0.001$ & $<0.001$ & 0.001 & 0.007 & $<0.002$ & $<0.002$ & $<0.004$ & 0.150 & 0.300 \\
\hline 46 & Eddy's Well & VA-49 & $<0.03$ & 0.113 & $<0.001$ & $<0.001$ & $<0.001$ & 0.021 & 0.117 & $<0.002$ & $<0.004$ & 0.288 & 0.040 \\
\hline 34 & Cold Mineral Seep & VA-50 & $<0.03$ & 0.022 & $<0.001$ & $<0.001$ & $<0.001$ & $<0.001$ & $<0.002$ & $<0.002$ & 0.016 & 0.810 & $<0.002$ \\
\hline 29 & Jemez River & VA-52 & $<0.03$ & 0.021 & $<0.001$ & $<0.001$ & 0.001 & 0.016 & $<0.002$ & $<0.002$ & $<0.004$ & 0.038 & 0.036 \\
\hline 48 & Unnamed cold Spring & VA-54 & $<0.03$ & 0.065 & $<0.001$ & $<0.001$ & 0.002 & $<0.001$ & $<0.002$ & $<0.002$ & $<0.004$ & 0.064 & 0.007 \\
\hline 49 & Unnamed Cold Spring & YA-55 & $<0.03$ & 0.074 & $<0.001$ & $<0.001$ & 0.005 & $<0.001$ & $<0.002$ & $<0.002$ & $<0.004$ & 0.043 & 0.015 \\
\hline 50 & Unnamed Cold Spring & VA-56 & $<0.03$ & 0.018 & $<0.001$ & $<0.001$ & 0.002 & $<0.001$ & $<0.002$ & $<0.002$ & $<0.004$ & 0.067 & 0.018 \\
\hline 51 & Apache Spring & VA-57 & $<0.03$ & 0.066 & $<0.001$ & $<0.001$ & 0.002 & $<0.001$ & $<0.002$ & $<0.002$ & $<0.004$ & 0.064 & 0.020 \\
\hline 52 & Unnamed Cold Spring & VA-58 & $<0.03$ & 0.016 & $<0.001$ & $<0.001$ & $<0.001$ & $<0.001$ & $<0.002$ & $<0.002$ & $<0.004$ & 0.049 & 0.024 \\
\hline 53 & Unnamed Cold Spring & VA-59 & $<0.03$ & 0.022 & $<0.001$ & $<0.001$ & 0.004 & $<0.001$ & $<0.002$ & $<0.002$ & $<0.004$ & 0.051 & 0.008 \\
\hline 54 & Turkey Springs & $V A-60$ & $<0.03$ & 0.056 & $<0.001$ & $<0.001$ & $<0.001$ & $<0.001$ & $<0.002$ & $<0.002$ & $<0.004$ & 0.066 & 0.080 \\
\hline 46 & Henson's Well & $Y A-61$ & $<0.001$ & 0.134 & $<0.001$ & $<0.002$ & $<0.001$ & 0.088 & 0.015 & $<0.001$ & $<0.004$ & 0.28 & 0.015 \\
\hline
\end{tabular}


TABLE B-III (cont)

\begin{tabular}{|c|c|c|c|c|c|c|c|c|c|c|c|c|c|}
\hline $\begin{array}{l}\text { Map } \\
\text { No. }\end{array}$ & Nane & $\begin{array}{l}\text { Field } \\
\text { Ho. }\end{array}$ & Ag & Ba & cd & Co & $\mathrm{Cr}$ & Cu & Mo & Ni & $\mathbf{P b}$ & Sr & $\mathrm{Zn}$ \\
\hline \multicolumn{14}{|c|}{ San Ysidro-Jemez Pueblo Area, Miscelloneous } \\
\hline 55 & Warm Mineral Spring & YA-33 & 0.006 & 0.044 & 0.006 & 0.016 & 0.008 & 0.009 & $<0.10$ & 0.002 & $<0.14$ & 7.70 & 0.001 \\
\hline 56 & Zia Hot Mell & VA-34 & 0.015 & 0.120 & 0.013 & 0.063 & 0.026 & 0.027 & $<0.10$ & 0.009 & 0.002 & 9.00 & $<0.01$ \\
\hline 56 & Zia Hot Hell & $v A-53$ & 0.006 & 0.028 & $<0.001$ & 0.004 & 0.004 & 0.020 & $<0.002$ & $<0.002$ & 0.360 & 4.75 & 0.007 \\
\hline 56 & Zla Hot Hell & $V A-67$ & $<0.001$ & 0.028 & $<0.001$ & 0.006 & 0.020 & $<0.001$ & $<0.002$ & $<0.001$ & 0.012 & 9.55 & $<0.002$ \\
\hline 57 & Unnamed Hell & $V A-35$ & -- & -- & -- & - & -- & -- & -- & -- & -- & $\cdots$ & -- \\
\hline 58 & Salt Spring & $V A-36$ & $<0.001$ & 0.032 & $<0.001$ & $<0.002$ & 0.004 & 0.016 & $<0.002$ & $<0.001$ & 0.008 & 4.82 & $<0.002$ \\
\hline 59 & Log Spring & VA-37 & -- & -- & -- & -- & -- & -- & -- & -- & -- & - & -- \\
\hline 60 & Owl Spring & $V A-38$ & $<0.001$ & 0.106 & $<0.001$ & $<0.002$ & $<0.001$ & $<0.001$ & 0.003 & $<0.001$ & $<0.004$ & 0.43 & 0.007 \\
\hline
\end{tabular}


TABLE B-IV

OXYGEN AND HYDROGEN ISOTOPE ANALYSES OF WATERS IN THE

JEMEZ MOUNTAINS REGION, NEW MEXICO

Map

Name Field No

Los Alamos Area, Pajarito Plateau

\begin{tabular}{|c|c|c|c|c|c|}
\hline 1 & Gallery Spring & $8 / 78$ & 11 & -84.3 & 12.20 \\
\hline 2 & T-3 Well & $8 / 78$ & 13 & -73.8 & -10.65 \\
\hline 3 & T-2 Well & $8 / 78$ & 11 & -73.5 & -10.60 \\
\hline 4 & Sacred Well & $8 / 78$ & 14 & -81.8 & -11.80 \\
\hline 5 & Basalt Spring & $8 / 78$ & 15 & -76.5 & -10.85 \\
\hline 6 & L-6 Wel1 & $9 / 78$ & 27 & -94.7 & -13.45 \\
\hline 7 & L-1B Well & $9 / 78$ & 30 & -103.0 & -14.30 \\
\hline 8 & L-5 We11 & $9 / 78$ & 26.5 & -- & -- \\
\hline 9 & L-4 We11 & $9 / 78$ & 28 & -- & -- \\
\hline 10 & PM-2 We11 & $9 / 78$ & 23.5 & -77.5 & -11.40 \\
\hline 11 & PM-1 Well & $9 / 78$ & 28 & -74.1 & -10.95 \\
\hline 12 & G-6 Well & $9 / 78$ & 30.5 & -76.0 & -11.25 \\
\hline 13 & G-5 Well & $9 / 78$ & 26.5 & -- & -- \\
\hline 14 & G-4 Well & $9 / 78$ & 26 & -76.3 & -11.10 \\
\hline 15 & G-3 Well & $9 / 78$ & 29 & -- & -- \\
\hline $16^{-}$ & G-2 Well & $9 / 78$ & 30 & -83.1 & -11.95 \\
\hline 17 & G-1A Well & $9 / 78$ & 28 & -82.5 & -11.80 \\
\hline 18 & G-1 Well & $9 / 78$ & 29 & -81.0 & -11.65 \\
\hline 19 & $\begin{array}{l}\text { Spring, White } \\
\text { Rock Canyon }\end{array}$ & $9 / 78$ & 19 & -76.8 & -11.00 \\
\hline 20 & PM-3 Well & $9 / 78$ & 27.5 & -- & -- \\
\hline
\end{tabular}


TABLE B-IV (cont)

Map

No.

Name

Field No. Collected

Temp

$80 \%$

$\delta^{18} 0 \%$

Valles Caldera, Sulphur Springs Area

\begin{tabular}{|c|c|c|c|c|c|c|}
\hline 21 & $\begin{array}{l}\text { Mudpot, Men's } \\
\text { Bathhouse }\end{array}$ & VA-13 & $1 / 79$ & 78 & -50.2 & -3.25 \\
\hline 21 & $\begin{array}{l}\text { Mudpot, Men's } \\
\text { Bathhouse }\end{array}$ & $S-7-80$ & $9 / 80$ & 82 & -- & -- \\
\hline 21 & $\begin{array}{l}\text { Unnamed Hot } \\
\text { Spring }\end{array}$ & VA-14 & $1 / 79$ & 63 & -60.7 & -8.80 \\
\hline 21 & $\begin{array}{l}\text { Unnamed Hot } \\
\text { Spring }\end{array}$ & $S-9-80$ & $9 / 80$ & -- & -- & -- \\
\hline 21 & Lemonade Spring & $S-10-80$ & $9 / 80$ & 58 & -- & -- \\
\hline 21 & Unnamed Spring & $S-3-80$ & $9 / 80$ & 11 & -- & -- \\
\hline 21 & Footbath Spring & $S-4-80$ & $9 / 80$ & 33 & -- & -- \\
\hline 21 & Electric Spring & $S-5-80$ & $9 / 80$ & 36 & -- & -- \\
\hline 21 & $\begin{array}{l}\text { Women's Bath- } \\
\text { house }\end{array}$ & $S-6-80$ & $9 / 80$ & 90 & -- & -- \\
\hline 22 & $\begin{array}{l}\text { Spring, Alamo } \\
\text { Canyon }\end{array}$ & $S-1-80$ & $9 / 80$ & 11 & -- & -- \\
\hline 22 & $\begin{array}{l}\text { Creek, Alamo } \\
\text { Canyon }\end{array}$ & $S-2-80$ & $9 / 80$ & 11 & -- & -- \\
\hline 23 & Bubbling Pool & VA-22 & $3 / 79$ & 0.5 & -97.3 & -13.45 \\
\hline 23 & Bubbling Seep & $V A-23$ & $3 / 79$ & 7 & -- & -- \\
\hline 24 & $\begin{array}{l}\text { Spring, Short } \\
\text { Canyon }\end{array}$ & $S-8-80$ & $9 / 80$ & 8 & -- & -- \\
\hline
\end{tabular}

Valles Caldera, Thermal Meteoric (Ring Fracture Zone)

$\begin{array}{llllllc}25 & \text { Spence Hot Spring } & \text { VA-1 } & 7 / 78 & 45 & -86.4 & -12.35 \\ 25 & \text { VA-2 } & 7 / 78 & 34 & -- & - \\ \begin{array}{l}\text { Little Spence } \\ \text { Hot Spring }\end{array} & \text { VA-3 } & 7 / 78 & 31 & -88.4 & -12.60\end{array}$


TABLE B-IV (cont)

Map

No.

Name

Date
Field No. Collected

Temp

$80 \%$

${ }_{\delta} 180 \%$

$27 \quad$ San Antonio

Hot Spring

VA-4

$7 / 78$

42

$-92.0$

$-12.65$

28 Bathhouse Spring

VA-20

$2 / 79$

38

$-86.4$

$-12.40$

Valles Caldera, Soda Dam and Jemez Springs (Jemez Fault Zone)

\begin{tabular}{|c|c|c|c|c|c|c|}
\hline 29 & Soda Dam Spring & VA-6 & $7 / 78$ & 47 & -84.9 & -10.60 \\
\hline 29 & Soda Dam Spring & VA-9 & $1 / 79$ & 48 & -- & -- \\
\hline 29 & Soda Dam Spring & VA-64 & $12 / 80$ & 47 & -85.2 & -10.60 \\
\hline 29 & Soda Dam Spring & VA-26 & $5 / 79$ & 47 & -- & -- \\
\hline 29 & Soda Dam Spring & $V A-51$ & $4 / 80$ & 47 & -85.4 & -10.70 \\
\hline 29 & Grotto Spring & VA-5 & $7 / 78$ & 38 & -84.6 & -10.65 \\
\hline 29 & $\begin{array}{l}\text { Outfall of } \\
\text { Soda Dam Spring }\end{array}$ & VA-65 & $12 / 80$ & 17 & -- & -- \\
\hline 29 & Unnamed Spring & VA-27 & $5 / 79$ & 29 & -84.9 & -10.95 \\
\hline 30 & Main Jemez Spring & VA-10 & $1 / 79$ & 55 & -82.3 & -10.6 \\
\hline 30 & Main Jemez Spring & $V A-18$ & $1 / 79$ & 36 & -81.4 & -10.4 \\
\hline 30 & $\begin{array}{l}\text { Travertine } \\
\text { Mound Spring }\end{array}$ & VA-7 & $1 / 79$ & 70 & -83.6 & -11.30 \\
\hline 30 & $\begin{array}{l}\text { Travertine } \\
\text { Mound Spring }\end{array}$ & VA-66 & $12 / 80$ & 72 & -83.1 & -11.35 \\
\hline 30 & $\begin{array}{l}\text { Travertine } \\
\text { Mound Spring }\end{array}$ & VA-17 & $1 / 79$ & 72 & -- & -- \\
\hline 30 & Buddhist Spring & $V A-8$ & $1 / 79$ & 49 & -- & -- \\
\hline 30 & Buddhist Spring & $V A-16$ & $1 / 79$ & 50 & -- & -- \\
\hline 30 & Unnamed Spring & VA-12 & $1 / 79$ & 49 & -- & -- \\
\hline 30 & $80 \mathrm{Ft}$ Aquifer & VA-19 & $1 / 79$ & 68 & -84.0 & -11.3 \\
\hline 30 & $80 \mathrm{Ft}$ Aquifer & VA-25 & $5 / 79$ & 73.3 & -- & -- \\
\hline
\end{tabular}


TABLE B-IV (cont)

Map

Name

Field No. Collected

No.

Temp

$30,500 \mathrm{Ft}$ Aquifer

VA-15

$1 / 79$

$60.5 \quad-85.9$

$-11.8$

$30 \quad 500 \mathrm{Ft}$ Aquifer

VA-21

$2 / 79$

61

Valles Caldera Region, Miscellaneous

\begin{tabular}{|c|c|c|c|c|c|c|}
\hline 28 & San Antonio Creek & VA-24 & $5 / 79$ & 2 & -92.9 & -12.85 \\
\hline 31 & Panorama Spring & $V A-28$ & $5 / 79$ & 13 & -86.9 & -11.80 \\
\hline 32 & Pajarito Spring & $V A-29$ & $7 / 79$ & 20 & -74.5 & -10.90 \\
\hline 33 & $\begin{array}{l}\text { Spring, White } \\
\text { Rock Canyon }\end{array}$ & $V A-30$ & $7 / 79$ & 18 & - & -- \\
\hline 34 & Cold Mineral Seep & VA-31 & $8 / 79$ & 19 & -92.9 & -12.50 \\
\hline 35 & Sino Spring & $V A-32$ & $8 / 79$ & 21 & -88.0 & -12.30 \\
\hline 35 & Sino Spring & $V A-63$ & $12 / 80$ & 18 & - & - \\
\hline 36 & Unnamed Spring & VA-39 & $8 / 79$ & 16.8 & -84.6 & -12.35 \\
\hline 37 & Canon Spring & $V A-40$ & $8 / 79$ & 20.2 & -86.6 & -12.35 \\
\hline 38 & Indian Valley Well & $V A-41$ & $\begin{array}{l}8 / 79 \\
10 / 79\end{array}$ & $\overline{17.5}$ & $\begin{array}{l}-87.1 \\
-91.1\end{array}$ & $\begin{array}{l}-12.30 \\
-12.75\end{array}$ \\
\hline 39 & Unnamed Cold Spring & $V A-42$ & $8 / 79$ & 15 & -85.0 & -12.40 \\
\hline 40 & Unnamed Cold Spring & $V A-43$ & $8 / 79$ & 14.8 & -78.2 & -11.40 \\
\hline 41 & Unnamed Cold Spring & $V A-44$ & $8 / 79$ & 9.8 & -98.2 & -13.75 \\
\hline 42 & Horseshoe Spring & VA-45 & $8 / 79$ & 14 & -90.2 & -12.65 \\
\hline 43 & Unnamed Cold Spring & $V A-46$ & $8 / 79$ & 13.3 & -96.5 & -13.60 \\
\hline 44 & Unnamed Cold Spring & VA-47 & $8 / 79$ & 12 & -99.1 & -14.25 \\
\hline 45 & Unnamed Cold Spring & $V A-48$ & $6 / 80$ & 9 & -- & -- \\
\hline 46 & Eddy's Well & VA-49 & $6 / 80$ & 15 & -- & -- \\
\hline 34 & $\begin{array}{l}\text { Cold Mineral } \\
\text { Seep }\end{array}$ & $V A-50$ & $4 / 80$ & 11 & -92.7 & -12.80 \\
\hline
\end{tabular}


TABLE B-IV (cont)

\begin{tabular}{|c|c|c|c|c|c|c|}
\hline $\begin{array}{l}\text { Map } \\
\text { No. }\end{array}$ & Name & Field No. & $\begin{array}{c}\text { Date } \\
\text { Collected }\end{array}$ & $\begin{array}{l}\text { Temp } \\
{ }^{\circ} \mathrm{C}\end{array}$ & $80 \%$ & $\delta^{18} 0^{\circ} \%$ \\
\hline 29 & Jemez River & $V A-52$ & $4 / 80$ & 5 & -94.4 & -13.20 \\
\hline 48 & Unnamed Cold Spring & VA-54 & $6 / 80$ & 11 & -88.6 & -12.60 \\
\hline 49 & Unnamed Cold Spring & VA-55 & $6 / 80$ & 8.5 & -90.5 & -12.75 \\
\hline 50 & Unnamed Cold Spring & VA-56 & $6 / 80$ & 6.5 & -87.6 & -12.55 \\
\hline 51 & Apache Spring & $V A-57$ & $7 / 80$ & 9 & -85.1 & -12.25 \\
\hline 52 & Unnamed Cold Spring & $V A-58$ & $7 / 80$ & 15 & -80.5 & -11.55 \\
\hline 53 & Unnamed Cold Spring & VA-59 & $7 / 80$ & 17 & -80.1 & -11.60 \\
\hline 54 & Turkey Springs & $V A-60$ & $7 / 80$ & 18 & -76.0 & -11.00 \\
\hline 46 & Henson's Well & $V A-61$ & $12 / 80$ & 19 & -81.2 & -11.30 \\
\hline
\end{tabular}

San Ysidro - Jemez Pueblo Area, Miscellaneous

$\begin{array}{llllllr}55 & \text { Warm Mineral Spring } & \text { VA-33 } & 8 / 79 & 27 & -86.6 & -10.40 \\ 56 & \text { Zia Hot Well } & \text { VA-34 } & 8 / 79 & 56 & -89.8 & -11.25 \\ 56 & \text { Zia Hot Well } & \text { VA-53 } & 4 / 80 & 54 & -89.0 & -12.55 \\ 56 & \text { Zia Hot Well } & \text { VA-67 } & 3 / 81 & 53 & -- & -- \\ 57 & \text { Unnamed Well } & \text { VA-35 } & 8 / 79 & 21 & -101.4 & -13.65 \\ 58 & \text { Salt Spring } & \text { VA-36 } & 8 / 79 & 29.2 & -84.9 & -10.20 \\ 59 & \text { Log Spring } & \text { VA-37 } & 8 / 79 & 28.5 & -87.7 & -12.25 \\ 60 & \text { Owl Spring } & \text { VA-38 } & 8 / 79 & 17.6 & -86.2 & -12.15\end{array}$




\section{REFERENCES}

1. F. E. Goff and C. 0. Grigsby, "Valles Caldera Geothermal Systems, New Mexico, USA," J. Hydrol., in press (1982).

2. F. E. Goff, C. O. Grigsby, P. E. Trujillo, Jr., D. Counce, and A. Kron, "Geology, Water Geochemistry and Geothermal Potential of the Jemez Springs Area, Cañon de San Diego, New Mexico," J. Volc. Geotherm. Res. 10, 227-244 (1981).

3. F. E. Goff and S. Sayer, "A Geothermal Investigation of Spring and Well Waters of the Los Alamos Region, New Mexico," Los Alamos Scientific Laboratory report LA-8326-MS, 21 pp. (April 1980).

4. F. W. Trainer and F. P. Lyford, "Geothermal Hydrology in the Rio Grande Rift, North Central New Mexico," New Mexico Geor. Soc., 30th Field Conf. Guidebook, 299-306 (1979).

5. F. W. Trainer, "Ground-Water in the Southwestern part of the Jemez Mountains Volcanic Region, New Mexico," New Mexico Geol. Soc., 25th Field Conf. Guidebook, 337-345 (1974).

6. F. W. Trainer, "Mixing of Thermal and Nonthermal Waters in the Margin of the Rio Grande Rift, Jemez Mountains, New Mexico," New Mexico Geol. Soc., 26th Field Conf. Guidebook, 213-218 (1975).

7. F. B. Titus, Jr., "Ground-Water Geology of the Rio Grande Trough in NorthCentral New Mexico, with Sections on the Jemez Caldera and the Lucero Uplift," New Mexico Geol. Soc., 12th Field Conf. Guidebook, 186-192 (1961).

8. W. D. Purtymun and S. Johansen, "General Geohydrology of the Pajarito Plateau," New Mexico Geol. Soc., 25th Field Conf. Guidebook, Ghost Ranch, 347-349 (1974).

9. W. D. Purtymun, F. G. West, and W. H. Adams, "Preliminary Study of the Quality of Water in the Drainage Area of the Jemez River and Rio Guadalupe," Los Alamos Scientific Laboratory report LA-5595-MS, 26 pp. (Apri1 1974).

10. W. D. Purtymun, R. J. Peters, and J. W. Owens, "Geohydrology of White Rock Canyon of the Rio Grande from Otowi to Frijoles Canyon," Los Alamos Scientific Laboratory report LA-8635-MS, 15 pp. (December 1980).

11. W. D. Purtymun, "Hydrologic Characteristics of the Los Alamos Well Field, With Reference to the Occurrence of Arsenic in Well L-6," Los Alamos Scientific Laboratory report LA-7012-MS, 63 pp. (November 1977).

12. C. S. Ross, R. L. Smith, and R. A. Bailey, "Outline of the Geology of the Jemez Mountains, New Mexico," New Mexico Geol. Soc., 12th Field Conf. Guidebook, 139-143 (1961). 
13. R. L. Smith, R. A. Bailey, and C. S. Ross, "Structural Evolution of the Valles Caldera, New Mexico, and Its Bearings on the Emplacement of Ring. Dikes," U.S. Geol. Surv., Prof. Paper 424-D, D145-D149 (1961).

14. R. L. Griggs, "Geology and Ground-Water Resources of the Los Alamos Area, New Mexico," U.S. Geol. Surv. Water-Supply Paper 1753, 107 pp.

15. R. R. Doell, G. B. Dalrymple, R. L. Smith, and R. A. Bailey, "Paleomagnetism, Potassium-Argon Ages, and Geology of Rhyolites and Associated Rocks of the Valles Caldera, New Mexico," Geol. Soc. Am., Mem. 116, 211-248 (1968).

16. R. A. Bailey, R. L. Smith, and C. S. Ross, "Stratigraphic Nomenclature of Volcanic Rocks in the Jemez Mountains, New Mexico," U.S. Geol. Surv. Bul1. 1274-P, 19 pp. (1969).

17. R. L. Smith, R. A. Bailey, and C. S. Ross, "Geologic Map of the Jemez Mountains, New Mexico," U.S. Geol. Surv. Misc Geol. Investig. Map I-571 (1970).

18. E. B. Mayo, "Lineament tectonics and some ore districts of the Southwest," Trans. AIME, 211, 1169-1175 (1958).

19. A. W. Laughlin, D. G. Brookins, and P. E. Damon, "Late-Cenozoic Basaltic Volcanism Along the Jemez Zone of New Mexico and Arizona," Geol. Soc. Am. Abst. w/Programs, $\underline{8}, 598$ (1976).

20. A. W. Laughlin, "The Geothermal System of the Jemez Mountains and Its Exploration," in Geothermal Systems - Principles and Case Histories, L. Rybach and L. J. P. Muffler, Eds. (John Wiley and sons, Inc., 1981) pp. 295-320.

21. R. A. Barnes, "The Determination of Specific Forms of Aluminium in Natural Water," Chem. Geol. 15, 177-191 (1975).

22. D. E. White, "Magmatic, Connate, and Metamorphic Waters," Geol. Soc. Am. Bull., 68, 1659-1682 (1957).

23. P. H. Craig, "Isotopic Variations in Meteoric Waters," Science, 133, 17021703 (1961).

24. W. K. Summers, "Catalog of Thermal Waters in New Mexico," New Mexico Bur. Mines. Miner. Resour. Hydrol. Rep., 4, 80 pp. (1976).

25. D. E. White, L. P. J. Muffler, and A. H. Truesdell, "Vapor-Dominated Hydrothermal Systems Compared With Hot-Water Systems," Econ. Geol., 66, 75-97 (1971).

26. R. H. Mariner, T. S. Presser, and W. C. Evans, "Chemical, Isotopic, and Gas Compositions of Selected Thermal Springs in Arizona, New Mexico and Utah," U.S. Geol. Surv., Open file report 77-654, 42 pp. (1977).

27. W. D. Purtymun, "Water Supply at Los Alamos During 1978," Los. Alamos Scientific Laboratory progress report LA-8074-PR, 46 pp. (October 1979). 
28. W. D. Purtymun and J. B. Cooper, "Development of Ground-water Supplies on the Pajarito Plateau," U.S. Geol. Surv., Prof. Paper 650-B, B149-B153 (1969).

29. F. W. Trainer, "Geohydrologic Data From the Jemez Mountains and Vicinity, North-Central New Mexico," U.S. Geol. Surv., Water-Resour. Invest., 77-131 146 pp. (1978). 
